Florida International University

FIU Digital Commons

FIU Electronic Theses and Dissertations

University Graduate School

$10-23-2020$

\title{
Investigations of Cell-Penetrating and Membrane-Pore Forming Peptides
}

Rudramani Pokhrel

Florida International University, rpokh002@fiu.edu

Follow this and additional works at: https://digitalcommons.fiu.edu/etd

Part of the Atomic, Molecular and Optical Physics Commons, and the Biological and Chemical Physics Commons

\section{Recommended Citation}

Pokhrel, Rudramani, "Investigations of Cell-Penetrating and Membrane-Pore Forming Peptides" (2020). FIU Electronic Theses and Dissertations. 4592.

https://digitalcommons.fiu.edu/etd/4592

This work is brought to you for free and open access by the University Graduate School at FIU Digital Commons. It has been accepted for inclusion in FIU Electronic Theses and Dissertations by an authorized administrator of FIU Digital Commons. For more information, please contact dcc@fiu.edu. 


\title{
FLORIDA INTERNATIONAL UNIVERSITY \\ Miami, Florida
}

\section{INVESTIGATIONS OF CELL-PENETRATING AND MEMBRANE-PORE FORMING PEPTIDES}

A dissertation submitted in partial fulfillment of the requirements for the degree of DOCTOR OF PHILOSOPHY

\author{
in \\ PHYSICS \\ by \\ Rudramani Pokhrel
}


To: Dean Michael R. Heithaus

College of Arts, Sciences and Education

This dissertation, written by Rudramani Pokhrel, and entitled Investigations of CellPenetrating and Membrane-Pore Forming Peptides, having been approved in respect to style and intellectual content, is referred to you for judgment.

We have read this dissertation and recommend that it be approved.

$\begin{array}{r}\hline \text { Xuewen Wang } \\ \hline \text { Jaroslava Miksovska } \\ \hline \text { Bernard S. Gerstman, Co-Major Professor } \\ \hline \text { Prem P. Chapagain, Co-Major Professor }\end{array}$

Date of Defense: October 23, 2020

The dissertation of Rudramani Pokhrel is approved.

Dean Michael R. Heithaus College of Arts, Sciences and Education

Andrés G. Gil

Vice President for Research and Economic Development and Dean of the University Graduate School

Florida International University, 2020 
(C) Copyright 2020 by Rudramani Pokhrel

All rights reserved. 


\section{DEDICATION}

To my parents; Top Ram and Mina Devi Pokhrel. My Ph.D. degree is his dream and would not have been possible without her sacrifice. 


\section{ACKNOWLEDGMENTS}

I want to express my profound gratitude and appreciation to my $\mathrm{Ph} . \mathrm{D}$. supervisors, Professor Prem P. Chapagain and Professor Bernard S. Gerstman. Their invaluable mentoring and support have provided me the necessary pieces of training to excel in my research. During my graduate years, I have never felt distressed or frustrated, mainly because of their patience, encouragement, and understanding. I am very grateful to my committee members Professor Xuewen Wang and Professor Jaroslava Miksovska, for their expert suggestions, comments, and willingness to help me always. I am thankful to the Department of Physics Faculty and Staff members, and my present and past lab members Tim, Jeevan, Nisha, Prabin, Lokman, Chandra, Elumalai for their suggestions and help in my research projects.

I want to express sincere regards to Professor Robert Stahelin and Professor Ras B Pandey for their collaboration in the Ebola virus protein VP40 projects. I am also grateful to Professor Jessica Liberles for her collaboration in the Corona Virus protein RdRP project. I am thankful to Barry Branch, the CASTIC team, and FIU HPC team for their computer support. I am grateful to Dr. Martin Handfield and Dr. Jae Park from Oragenics Biopharmaceuticals for collaboration and supporting my research assistantship for a semester. I greatly appreciate and express my acknowledgment to the University Graduate School at Florida International University for awarding me the Dissertation Year Fellowship (DYF) award. This award helps me a lot to complete my research projects and dissertation writing.

I owe more than an acknowledgment to my parents for the continuous love and support. I always remember the support, encouragement, and care of my brothers, sisters, 
and friends towards my long education journey. I am forever indebted to my brother Bishnu Pokhrel, for his moral and financial support throughout my student life from my early campus days. My beloved wife, Sushila Bhandari, has a unique place in this acknowledgment for providing unbound love, care, and understanding. There is no match or compensation for her sacrifice to support me and to rear two children simultaneously. She is always aware that I should get enough time to do my research work and keep me out of household activities. I want to thank my in-laws for their love, support, and suggestions. Finally, I am incredibly fortunate to have two baby angles Parth and Ojas. At the end of the day, their welcome smile always instantly reinvigorates me. 


\section{ABSTRACT OF THE DISSERTATION \\ INVESTIGATIONS OF CELL-PENETRATING AND MEMBRANE-PORE FORMING \\ PEPTIDES}

by

Rudramani Pokhrel

Florida International University, 2020

Miami, Florida

Professor Bernard S. Gerstman, Co-Major Professor

Professor Prem P. Chapagain, Co-Major Professor

Cell-penetrating and membrane-pore forming peptides are a class of membraneactive peptides. They are short sequence peptides having both hydrophilic and lipophilic combinations of amino acids. These peptides can have contrast functional diversity. Peptides encoded by viruses function as viroporins and play a critical role in viral replication, propagation, and pathogenesis. One such peptide is the Ebola virus delta peptide, which forms a pore in the host cell membrane. Another set of pore-forming peptides are antimicrobial Lantibiotic peptides that may be useful for killing antibiotic resistant bacteria by disrupting the bacterial membrane through two different possible mechanisms. Not only can they form pores that disrupt the bacterial membrane, Lantibiotics can also hinder the function of a bacterial membrane molecule, Lipid II, that is essential for cell wall biosynthesis.

I performed Molecular Dynamics (MD) simulation to investigate the membrane pore-forming mechanism of the Ebola virus delta peptide. I found that the Ebola virus delta peptide forms a pentameric pore in the host cell membrane. The pore is selective for 
negatively charged ions, and the disulfide link between Cysteine 29 and 38 is essential for stable and effective membrane-pore formation. Similarly, I studied Lipid II binding and membrane-pore forming activity of several Lantibiotics: Nisin, Mutacin 1140, Gallidermin, NAI107, and NVB302. The computational results from MD simulations show that Nisin forms the most effective water channel in the bacterial membrane, but Gallidermin has the best Lipid II binding profile. Mutacin 1140 also binds strongly with Lipid II and forms an efficient water channel in the bacterial membrane. I also performed MD computations on a variety of mutated peptides of Mutacin 1140 and the Ebola virus delta peptides and investigated their water solubility, Lipid II binding, and membrane insertion profile. These results provide insight into the possible antimicrobial peptides with an optimized drug profile. 
TABLE OF CONTENTS

CHAPTER

PAGE

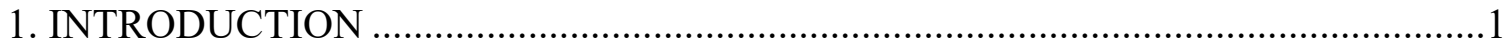

1.1 Cell-Penetrating and Membrane Pore-Forming Peptides …………............................

1.1.1 Ebola Virus Delta Peptide............................................................................. 4

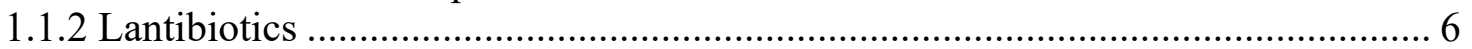

1.2 Molecular Dynamics Computer Simulation ..........................................................

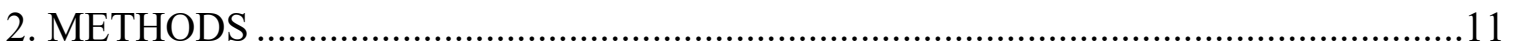

2.1 All Atom Molecular Dynamics (AAMD) Simulation Protocol...................................11

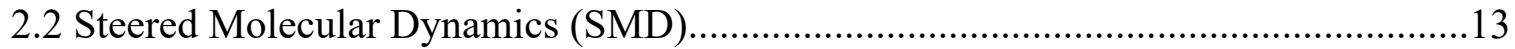

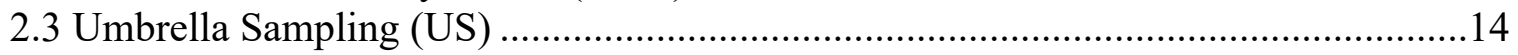

3. MEMBRANE PORE FORMATION AND ION SELECTIVITY OF THE EBOLA

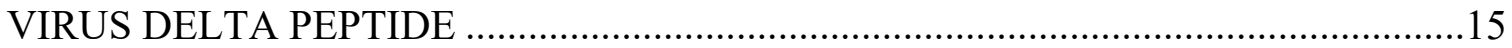

3.1 Construction of the Transmembrane Assembly......................................................15

3.2 Delta-Peptide Assembly and Dynamics.................................................................. 17

3.3 Hydrogen Bond and Interaction Energy Profiles in Various Pore Assemblies ...........20

3.4 Pore Size, Water and Ion Fluxes............................................................................21

3. 5 Role of the Disulfide-Bond in Pore Formation and Ions Flux .................................25

4. LIPID II BINDING AND TRANSMEMBRANE PROPERTIES OF

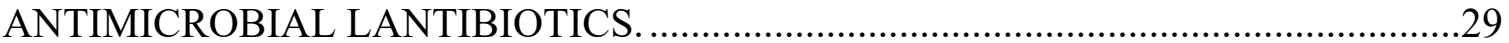

4.1 Molecular Mechanisms of Pore Formation and Membrane Disruption by the Antimicrobial Lantibiotic Peptide Mutacin 1140 ............................................................29

4.1.1 Structural Comparison of MU1140 and Nisin ................................................. 29

4.1.2 MU1140 Interactions with The Membrane Surface and Lipid II ........................ 31

4.1.3 Membrane Insertion of MU1140 ………………...................................... 34

4.1.4 Membrane Pore Formation and Water Permeation by MU1140 …..................... 35

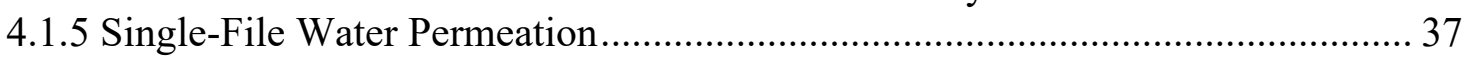

4.1.6 Water Permeation in Multimeric Assembly of MU1140-Lipid II Complexes .... 41

4.2 A Comparative Study of The Lipid II Binding and Transmembrane Properties of

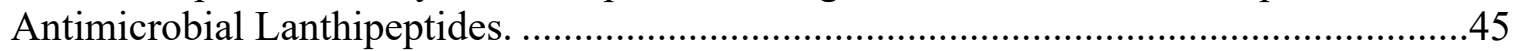

4.2.1 Molecular Models of Peptide-Lipid II Complexes ........................................... 46

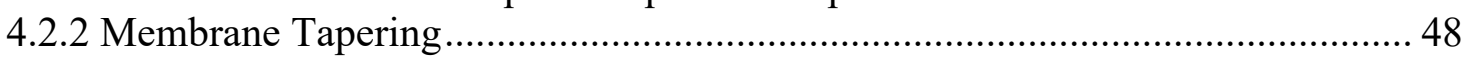

4.2.3 Channel Formation: Insertion and Transport of Water Across the Membrane ... 50

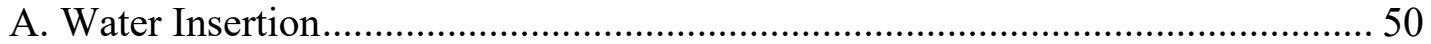

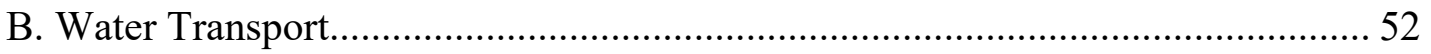

C. Peptide Hydrogen Bonding with Water .............................................................. 53

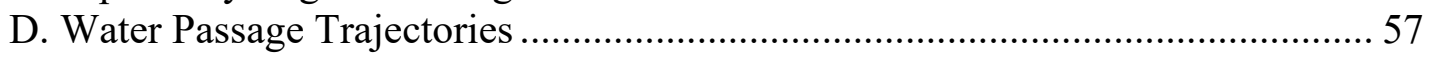

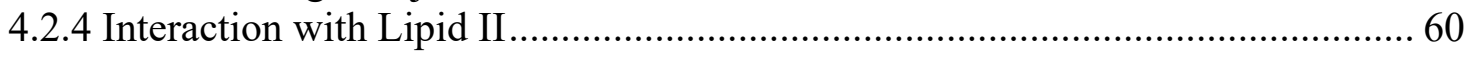

5. DESIGNING HYBRID LANTHIPEPTIDES FROM MUTACIN 1140 AND EBOLA VIRUS DELTA PEPTIDE FOR IMPROVED DRUG PROFILE 


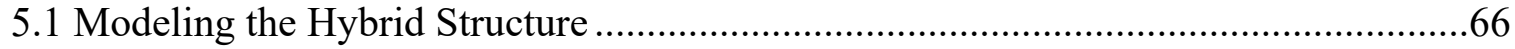

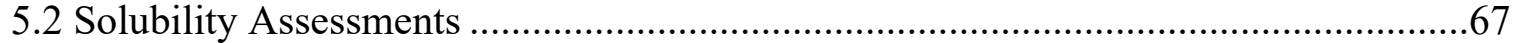

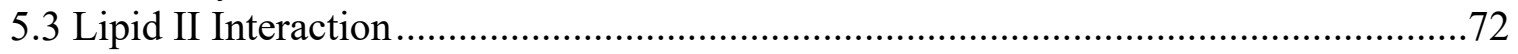

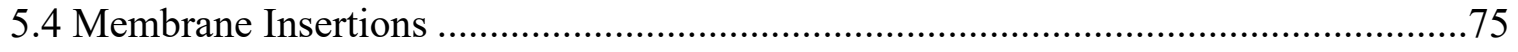

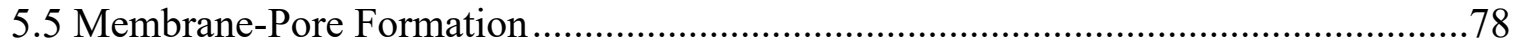

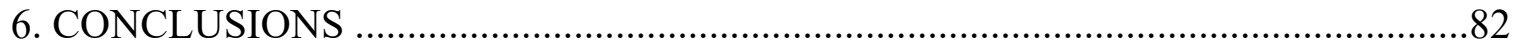

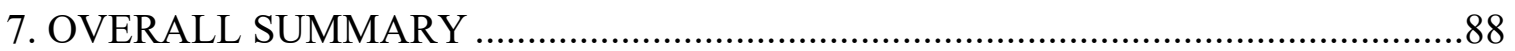

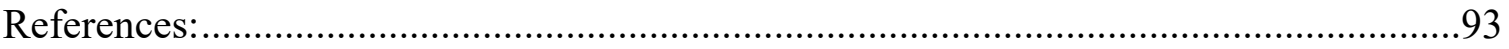

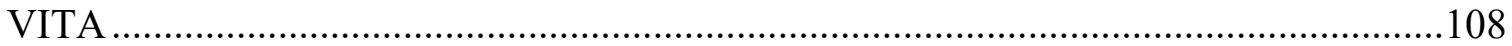




\section{LIST OF FIGURES}

FIGURES

PAGES

Figure 1.1 The primary structure of the Ebola virus delta peptide and its 3D-structure predicted by Phyre236. Color coding: basic residues (gray-blue), acidic residues (pink), hydrophobic (white), hydrophilic (green), and glycosylated sugar (orange). The disulfide bond between C-29 and C-38 is shown in yellow.

Figure 1.2 The primary sequence of a lantibiotic, Gallidermin. It has four sulfur bridged rings and first two rings serve as a lipid II binding moiety.

Figure 1. 3 Illustration of all bonded terms of potential energy used in MD

Figure 3.1 The initial structure of the delta peptide pore model for (a) tetramer (b) pentamer (c) hexamer. The N-ter of the peptides are placed at the inner surface of the membrane, which is at the bottom of the diagram. Peptides are shown by ribbon depiction. Color coding: basic residues-blue-gray, acidic residues-purple, membrane-transparent light blue cyan colors.

Figure 3.2 For tetrameric, pentameric, and hexameric pores: (a) Time evolution of the root mean square deviation (RMSD) from the initial position averaged over all $\mathrm{C} \alpha$, and (b) root mean square fluctuation (RMSF) in the position of each $\mathrm{C} \alpha$ averaged over the last $100 \mathrm{~ns}$.

Figure 3.3 Comparison of initial pore structure to pore structure at $500 \mathrm{~ns}$. The top and middle row are end views looking from outside the membrane and the bottom is a side view. Columns: (a) tetramer (b) pentamer and (c) hexamer. In the end views, Arg-39 is shown as gray-blue color.

Figure 3.4 Averaged non-bonded pore interactions: (a) electrostatic, (b) van der Waals, and (c) H-bonds

Figure 3.5 a) Volumetric map of the pores for various oligomeric assemblies. b) Average radius of pores calculated from the last $300 \mathrm{~ns}$ of the MD simulations. The volumetric maps and the radius profiles are colored the same way in a) and b).

Figure 3.6 Along the length (z-axis) of the pores, the relative density profile of: (a) water (b) chloride (c) sodium.

Figure 3.7 Flux of Cl- ions through the pores for (a) tetramer (b) pentamer (c) hexamer. The hexamer allows the largest flux.

Figure 3.8 For peptides without the disulfide bond in the C-ter region: (a) Initial and final configuration of SMD simulation of a single delta peptide in which the Arg39 side chain is pulled at constant velocity out of the membrane. (b) Free Energy 
of the peptide without the disulfide bond as a function of the C-ter tail relative to the surface of the membrane ( $\mathrm{z}=0$ ) (c) Pentamer pore model, (d) Hexamer pore model, in the bilayer membrane system.

Figure 3.9 For the pentamer pore and the hexamer pore, comparison of the RMSD for the peptides' $\mathrm{C} \alpha$ with and without peptide disulfide bonds. Here, curves for the peptides without the disulfide bond are labeled as WO-DISU and with the disulfide bond are labeled as W-DISU

Figure 3.10 a) Average pore radius from the last $300 \mathrm{~ns}$ of the MD simulation for the pentamer and (b) hexamer, composed of peptides with and without disulfide bonds. The shaded region shows the standard deviation of pore radius. The much wider fluctuations of the end residues at the C-ter ( $\mathrm{z}>20 \AA$ ) of the pores without peptide disulfide bonds reflects the decreased stability of the pore. c) Comparison of average density of chloride atoms along the pore for pores with and without peptide disulfide bonds for pentamer and (d) hexamer. For both the pentamer and the hexamer, fewer chloride ions are found in the pores without peptide disulfide bonds.

Figure 4.1 Schematic representation of amino acid sequences of (a) nisin and (b) MU1140 chains. The NMR structure of (c) the nisin-lipid II complex59 and d) the modeled structure of the MU1140-lipid II complex. The lantibiotic chains are shown in blue and lipid-II in orange. The phosphate and oxygen atoms in the pyrophosphate moiety are highlighted in gray and red respectively.

Figure 4.2 (a) MU1140-lipid II complex in the membrane at 0 ns and 500 ns. MU1140 is colored in by atom names, lipid II in orange, POPE in green, and POPG in gray. The phosphorous atoms in the lipid headgroups are shown as spheres. (b) Percentage hydrogen bonds between MU1140 and membrane as well as between MU1140 and lipid II (inset). The \% H-bond between MU1140 and lipid II are colored as, green: sidechain (SC), blue: backbone NH group, and red: backbone CO group. Important MU1140 residues participating in hydrogen bonding with lipid II are highlighted (right). c) Number of hydrogen bonds of MU1140 with lipid II as well as with other lipids in the membrane as a function of time. d) Number of lipid contacts with MU1140 as a function of time.

Figure 4.3 a) Insertion of MU1140 into the HMMM membrane. For clarity, DCLE molecules in the transmembrane region are not shown. b) Distance between the Tyr20 of MU1140 and the center-of-mass (com) of the lipid bilayer (membrane mid-point).

Figure 4.4 MU1140-lipid II complex in a bacterial membrane: a) Initial configuration of the complex and b) a configuration at the end of 500-ns simulation. The phosphorus atoms of POPE are highlighted as green spheres and that of POPG as gray sphere. The water molecules within $4 \AA$ of the peptide are highlighted as red spheres. 
Figure 4.5 a) A representative snapshot of the water permeation along the MU1140 chain (cyan) in the transmembrane region. Lipid II is shown in orange. b) Percentage of hydrogen-bonding between MU1140 residues and water molecules, showing MU1140 hydrogen bonds with backbone CO (red), backbone $\mathrm{NH}$ (blue), and sidechain (SC) (green). Only the phosphate atoms of the lipids are displayed for clarity.

Figure 4.6 a) MU1140 orientation in the lipid bilayer along z-axis b) Representative trajectories of successful water permeation across the membrane. c) A histogram of the number of water molecules along z-axis is shown on the side.

Figure 4.7 Assemblies of a) two and b) four complexes of MU1140-lipid II in the bacterial membrane at the beginning and end of the $500 \mathrm{~ns}$ simulations. MU1140 chains are colored by residue types, lipid II chains are highlighted in yellow, and the water molecules within $3.5 \AA$ of MU1140 are highlighted as red spheres. As in Figure 4.4, the phosphorus atoms of POPE are shown as green spheres and POPG phosphorous atoms as gray spheres. c) Same as b) but showing the water molecules above and below the lipid layers. The membrane is tapered near the MU1140-lipid II assembly due to the short length of MU1140. d) Density profile of the water molecules in the transmembrane region of the lipid bilayer for one, two, and four complexes of MU1140-lipid II.

Figure 4.8 Representative snapshots of a) two and b) four MU1140-lipid II complexes showing water permeation. MU1140 chains are shown in cyan color and lipid II chains are shown in orange. For clarity, only the phosphate atoms of the lipids are displayed. C) Percentage of hydrogen-bonding per chain for MU1140 and water in the transmembrane region for one, two, and four complexes of MU1140lipid II

Figure 4.9 (Left) Schematic representation of the amino acid sequence for the lantibiotic variants. (Right) Their modeled structure after a $10 \mathrm{~ns}$ MD simulation with lipid II (orange) in solution, with no membrane.

Figure 4.10 Snapshot of the protein-lipid II membrane complexes: (left) initial set-up, and (right) after $500 \mathrm{~ns}$ of MD computational simulation. The bacterial membrane is displayed in gray and the gray spheres are the phosphate atoms on the two surfaces of the lipid bilayers. Red spheres are water molecules within $5 \AA$ of the peptides.

Figure 4.11 Membrane thickness Average distance between phosphate atoms of the top and bottom of the membrane within $12 \AA$ of peptides.

Figure 4.12 For the protein-lipid II complexes: a) Density profile of the water molecules along the $z$-axis of the transmembrane region of the lipid bilayer, $b$ ) During the last $300 \mathrm{~ns}$ of the MD simulation, the time evolution of the number of $\mathrm{H}$-bonds made by each peptide with water molecules. 
Figure 4.13 For each of the peptide-lipid II complexes: a) Number of water molecules passing across the membrane from either direction summed over the last $300 \mathrm{~ns}$ of the MD simulation, b) Average passage time of a water molecule to cross the membrane region. "Up" and "Down" refers to the average position of the phosphate atoms of lipids in the lower and upper surfaces of the untapered membrane.

Figure 4.14 Percentage of hydrogen-bonding between peptide residues and water molecules, showing residual hydrogen bonds with backbone $\mathrm{CO}$ (red), backbone $\mathrm{NH}$ (blue), and sidechain (SC) (green).

Figure 4.15 Average time spent by water molecules near each residue (within $2.5 \AA$ )... 57

Figure 4.16 For the last $300 \mathrm{~ns}$ of the MD simulation: Column I) Average position of peptide (Cyan) and the phosphate atoms (gray) of the membrane surfaces. (II) Trajectories of all water molecules that successfully cross the membrane (colors represent distinct water molecules). (III) Two-dimensional density plot along the $\mathrm{x}-\mathrm{y}$ plane of the projection of the trajectories in Column II.

Figure 4.17 a) Total energy (Elec+VDW) of interaction between peptide and lipid II molecule. b) The number of $\mathrm{H}$-bonds made by each peptide with a lipid II molecule. c) Important residues (blue) in each peptide that make hydrogen bonds with the lipid II molecule (orange).

Figure 4.18 For the last $300 \mathrm{~ns}$ of the MD simulation, hydrogen bond occupancy percentage between lipid II molecules and the peptide residues highlighted in Fig. 4.17. Hydrogen bonds with peptide: backbone CO (red), backbone NH (blue), and side chain (green).

Figure 5.1 Hybrid model from MU1140 and the EBOV delta peptide with possible mutation. a) The shorter models S and SM. b) Medium models $\mathrm{M}$ and MM. c) Longer models L and LM. The mutated models SM, MM, and LM are from mutating Glu33 (orange star) with Arginine.

Figure 5.2 a) Snapshots of peptides form the initial (left of arrow) and final frames of 500 ns MD trajectories. The blue and red spheres represent the $\mathrm{N}$ and $\mathrm{C}$-terminal carbon atoms. The blue and green licorice structures represent the charged and polar amino acids while the white color represents the hydrophobic amino acids. b) Box plot of the average radius of gyration (RGYR) form the last $200 \mathrm{~ns}$ of the $\mathrm{MD}$ production run. The blue star represents the mean value.

Figure 5.3 Normalized plot of various parameters from Fig. 5.2: H-bonds (number of hydrogen bonds of peptide with water molecules), Hy-fluctuation (fluctuation of water molecules in first hydration layer of peptides, SASA-philic (SASA of hydrophilic amino acids), and SASA-phobic (SASA of hydrophobic amino acids). 
Figure 5.4 a) Snapshot of L variant initially placed at the surface of the membrane, and after $1 \mu$ s of MD simulation. The brown molecules are lipids in the membrane and the brown spheres are phosphate atoms of lipids in the membrane surface. For all six variants, the time evolution of: (b) number of hydrogen bonds, and (c) interaction energies between the peptide and lipid II molecules.

Figure 5.5 Percentage occupancy of hydrogen bonds between peptide amino acids and lipid II, averaged over the last $300 \mathrm{~ns}$ of $1 \mu \mathrm{s}$ MD run for each variant: (a) L, (b) LM, (c) M, (d) MM, (e) S, (f) SM.

Figure 5.6 a) Pictorial representation of the SMD constant velocity pulling through the membrane of backbone carbon atoms of three amino acids at the tail of variant $\mathrm{L}$. b) Force required to pull at constant velocity across the z-coordinate of the membrane. The two dotted vertical lines define the upper and lower boundaries of the lipid bilayer.

Figure 5.7 Free energy of pulling of peptides across the membrane............................ 77 


\section{ABBREVIATIONS AND ACRONYMS}

CHARMM Chemistry at Harvard Molecular Mechanics

CTD Carboxy Terminal Domain

LJ Lennard-Jones

MD Molecular Dynamics

NAMD Not Another Molecular Dynamics

NTD Amino Terminal Domain

PDB Protein Data Bank

PMF Potential of Mean Force

SASA Solvent Accessible Surface Area

US Umbrella Sampling 


\section{INTRODUCTION}

\subsection{Cell-Penetrating and Membrane Pore-Forming Peptides}

The membrane is a natural barrier that stabilizes and protects living cells by separating the cells' internal and external environments. It precisely controls the exchange of water, ions, and other nutrients between a cell and the extracellular environments. Though the thickness of a membrane is only about $5 \mathrm{~nm}{ }^{1}$, its structure and composition are very complex and diverse throughout different species. For example, a mammalian membrane is primarily composed of phospholipids, cholesterol, membrane proteins, carbohydrates, and fatty acids ${ }^{2}$, while the bacterial membrane is devoid of sterol and typically is negatively charged ${ }^{3}$. Despite these differences, the major components of both kinds of the cell membrane are the phospholipids. These lipids have hydrophilic head groups and hydrophobic alkyl chains ${ }^{4}$. The amphipathic nature of lipids enables them to assemble into the membrane complex in water readily. The morphology of the complex is either a lamellar bilayer or non-lamellar as in micelles ${ }^{5}$. In bilayer assemblies, hydrophilic head groups are exposed on the inner and outer membrane surface, and the long lipid molecules are aligned vertically (perpendicular to the membrane surface) so that the hydrophobic alkyl chains are buried in the membrane core and not exposed to water. The vertical alignment of the chains allows short amphipathic peptides to insert and penetrate these lipid bilayers.

Membrane-active peptides are short-sequence proteins that may or may not have a lytic effect on the cell membrane ${ }^{6}$. Most membrane-active peptides are amphipathic; however, they differ in their mode of actions on mammalian membranes compared to 
bacterial membranes ${ }^{7}$. The highly diverse class of membrane interacting peptides are antimicrobial peptides (AMPs). In multicellular organisms, AMPs are essential for innate immunity against invading pathogenic microorganisms ${ }^{8}$. The AMPs are positively charged short peptides; hence they engage actively with the generally negatively charged bacterial membrane. The amphipathic composition of amino acids provides solubility in both aqueous solution and membrane environment ${ }^{8}$. Their usual activity involves creating plasma membrane perturbation usually via membrane pore formation or membrane permeabilization, which causes cell lysis in pathogens ${ }^{9-10}$. A unique subgroup of AMPs produced by bacteria, called lantibiotics, hinders the cell wall synthesis of bacterial membranes by trafficking molecules that are important for the synthesis process. In recent studies, lantibiotics have shown great potential against the resistant-strains ${ }^{11-12}$. Because of their remarkable structural and functional diversity, AMPs offer a wide spectrum of activity and make them promising candidates for therapeutic applications ${ }^{13}$.

Recently, a new class of peptides encoded mainly by RNA viruses has been identified, which are critical for viral replication, propagation, and pathogenesis ${ }^{14}$. They are usually called viroporins, and more recently, these proteins have also been found in small DNA viruses ${ }^{14}$. They can alter the host cell membrane resulting in the disruption of ionic gradient, membrane fusion, budding, and release of virions ${ }^{15}$. These viroporins generally have at least one amphipathic alpha-helix and form oligomeric transmembrane channels in the plasma membrane or endoplasmic reticulum of the host cell. Some examples of viroporins are HIV-1 vpu, hepatitis C virus (HCV) $\mathrm{p} 7$ protein, and ebola virus delta-peptides ${ }^{15-17}$. Since they are important for the viral life cycle, viroporins are promising targets for clinical intervention ${ }^{18}$. There is another group of short peptides that 
can translocate through membrane bilayers without causing significant damage to membrane integrity and hence they are important for intracellular drug delivery in biological experiments. These are either arginine-rich peptides such as the TAT peptide or lysine-rich amphipathic peptides such as $\operatorname{Transportan}^{19}$.

The method in which these peptides penetrate and disrupt the cell membrane has been extensively explored experimentally over many decades and more recently using computational molecular dynamics (MD) simulations. However, an understanding of the mechanism at the molecular level is still lacking ${ }^{6,20-21}$. Also, the interaction mechanisms with the membrane of recently discovered peptides such as mutacin 1140 (MU1140) and Ebola virus delta-peptides are largely unknown. It is important to understand the modes of interaction of these peptides with cellular membranes so that we can devise inhibitors for cytotoxic peptides such as viroporins to prevent viral infection, and also learn how to modify these toxic peptides to selectively target cancer cells. On the other side, we would also like to strengthen AMPs to fight against the threat of antibiotic-resistant strains of bacteria.

My work focuses on two of the peptides highly worthy of investigation to address the current challenges in human health: the EBOV delta peptide and Lantibiotics. By using molecular dynamics simulation, for the first time, I have investigated the membrane poreforming mechanism of EBOV delta peptides, which may lead to the design and development of targets for pore modulators and disruptors, and thus the ability to controll virus propagation. Additionally, I have explored the bacterial killing mechanism of a few important lantibiotics, e.g., Mutacin 1140, Nisin, etc. and probed their efficacy for lipid II binding and membrane pore formation. My research also involves the computationally modeled hybrid peptides from the 
combination of MU1140 and EBOV delta peptide. I have investigated the water solubility and membrane insertion profiles of these hybrid peptides alongside with wild-type MU1140 to inform the peptide with the optimized antimicrobial profile. Such a detailed study at the atomic level could facilitate the design and development of antiviral and antibacterial regimens.

\subsubsection{Ebola Virus Delta Peptide}

The Ebola virus (EBOV), a member of the Filoviridae family, causes a severe hemorrhagic disease with an average current fatality rate of $\sim 50 \%$ in humans ${ }^{22}$. Of the five Ebola species that are identified within the genus Ebolavirus, four cause Ebola virus disease (EVD) in humans, while the fifth only causes a disease in nonhuman primates ${ }^{23}$. The Zaire Ebolavirus species was responsible for the major 2013 EVD outbreak that occurred in West Africa ${ }^{24-26}$. Ebola is especially dangerous because of a lack of effective vaccines and chemotherapeutics ${ }^{27-28}$, and underscores that EVD remains a major challenge in basic, translational, and clinical research. The elucidation of the molecular mechanisms responsible for EBOV replication and pathogenesis is an important step in the development of antiviral therapies.

Recent studies have shown that the Ebola virus delta peptide (Figure 1.1) ${ }^{29}$, a partially conserved non-structural protein, can act as a viroporin and thus may be involved in the pathology caused by $\mathrm{EVD}^{20,30}$. Viroporins are relatively small hydrophobic transmembrane proteins that are encoded by a broad range of viruses ${ }^{15,31-34}$, including $\mathrm{EBOV}^{20,30}$. The viroporin proteins homo-oligomerize in the host cell membrane to form amphipathic pores that modify the properties of intracellular membranes ${ }^{15,35}$. Importantly, the viroporins have become novel drug targets ${ }^{34}$ because of their numerous vital roles in the virus, such as membrane permeability ${ }^{36}$, ion flux, protein trafficking, and virion 
morphogenesis and release from host cells ${ }^{15}$. However, the molecular mechanisms of pore formation and the functioning of the Ebola virus delta peptide viroporin are not well understood.

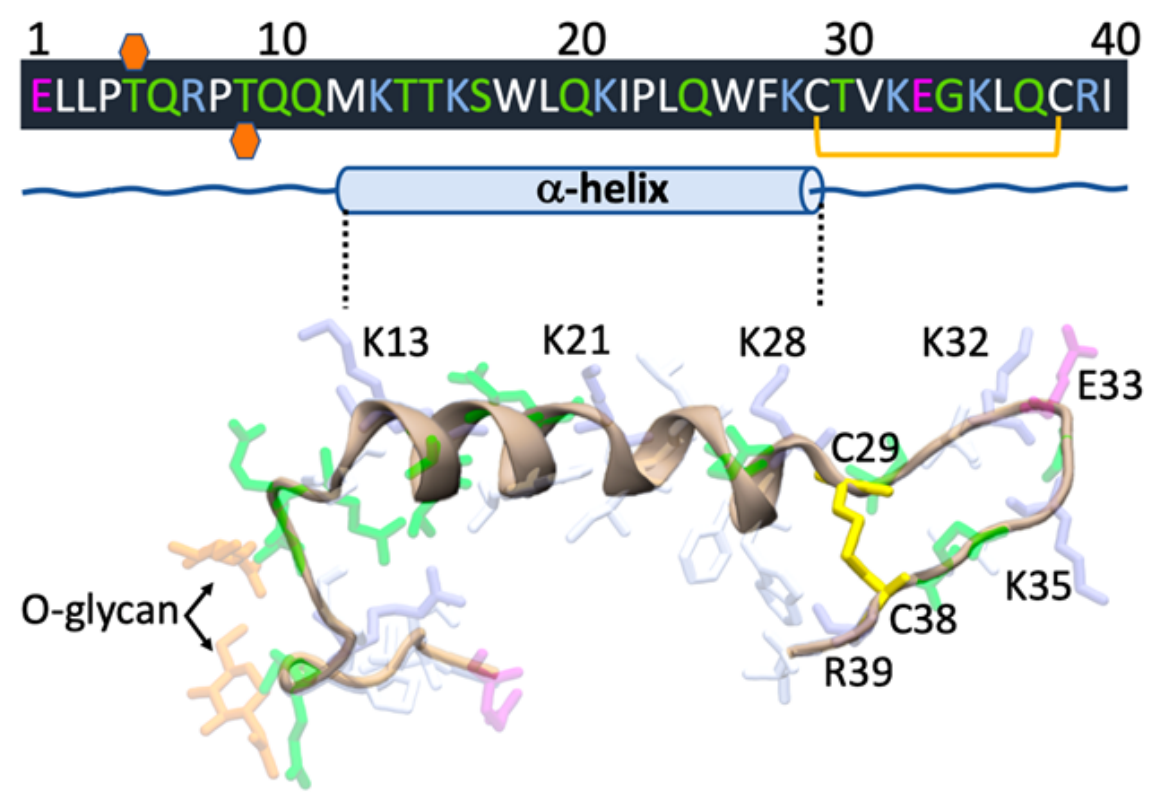

Figure 1.1 The primary structure of the Ebola virus delta peptide and its 3D-structure predicted by Phyre236. Color coding: basic residues (gray-blue), acidic residues (pink), hydrophobic (white), hydrophilic (green), and glycosylated sugar (orange). The disulfide bond between C-29 and C-38 is shown in yellow.

During the EVD, the delta peptide is produced from the cleavage of the carboxylterminal of the secreted glycoprotein (sGP) ${ }^{37-38}$. The 40 -residue long delta peptide has a variable 17 residue $\mathrm{N}$-terminus ( $\mathrm{N}$-ter) and a conserved, 23 residue $\mathrm{C}$-terminus $\left(\mathrm{C}\right.$-ter) ${ }^{20}$, ${ }^{30}$. The aromatic and basic amino acid rich motif, and the two cysteines at the $\mathrm{C}$-ter of the peptide, are conserved within all Ebola species, as well as in other pore forming peptides, including the cytolytic peptide of the non-structural protein 4 of the rotavirus ${ }^{39}$. Two aspects of the delta peptide are significant for its pore-forming ability ${ }^{20},{ }^{30}$, i) an amphipathic $\alpha$-helical structural motif in the middle that drives the membrane activity of 
many pore-forming peptides ${ }^{15,35,40}$, (ii) the conserved pair of cysteine residues (Cys-29 and Cys-38) in the C-ter that can help to form a disulfide cross-linked hairpin amphipathic structure that is similar to many membrane-permeabilizing antimicrobial peptides ${ }^{32}$. A recent experimental study demonstrated the presence of the C-ter, disulfide cross-linked hairpin structure for the membrane-permeabilizing activity ${ }^{41}$. The experimental study also showed that the delta peptide forms smaller and more selective ion-permeable pores in a synthetic lipid-bilayer.

\subsubsection{Lantibiotics}

In recent years, there has been an alarming rise in antibacterial resistance infections caused by inappropriate use of antibiotics in humans and agricultural feed ${ }^{42-45}$. Antibiotic resistance now accounts for higher medical costs, prolonged hospital stays, and increased mortality ${ }^{46}$. Globally, there is a high incidence rate of hospital-acquired infections, including infections by methicillin-resistant Staphylococcus aureus (MRSA) or multidrugresistant (pan-resistant) Gram-negative bacteria ${ }^{44,47-48}$. Several studies warn that if there is not a major advancement in antibiotics, there may be tens of millions of deaths annually along with trillions of dollars of medical expenses due to antibiotic resistant infections by the year $2050^{49-50}$. Hence there is a dire need for the development of new antibiotics utilizing novel modes of action. A unique class of peptides, lantibiotics are promising candidates to engage in the fight against resistant strains of bacteria ${ }^{51-53}$.

Lantibiotics are ribosomally synthesized peptides that contain post-translationally modified unusual amino acids, such as lanthionine (Lan), ß-methyl-lanthionine (Melan), didehydroalanine (Dha), didehydrobutyrine (Dhb) and aminovinyl-cysteine (Avicys) ${ }^{52,}$ 5455 (Figure 1.2). A thioether ring Lan (Ala-S-Ala) is formed when the B-carbons of two 
alanine-like derivatives cross-linked via a sulfur atom. Likewise, Melan (Abu-S-Ala) is formed between alanine and aminobutyric acid-like derivatives. On the other hand, AviCys is decarboxylated Lan which is located at the last position of lantibiotic sequence. The post translationally modified aminoacids Dha and Dhb are derived from dehydration of serine and threonine residues in the precursor peptides ${ }^{56-57}$. The two main categories of high activity lantibiotics, Type A and Type B, are distinguished by their biosynthesis pathways and structures ${ }^{58}$. Type A lantibiotics are longer and more flexible than Type B, which include nisin, MU1140, gallidermin and epidermin. The shorter, globular Type B lantibiotics include mersacidin, cinnamycin, and duramycins ${ }^{59}$

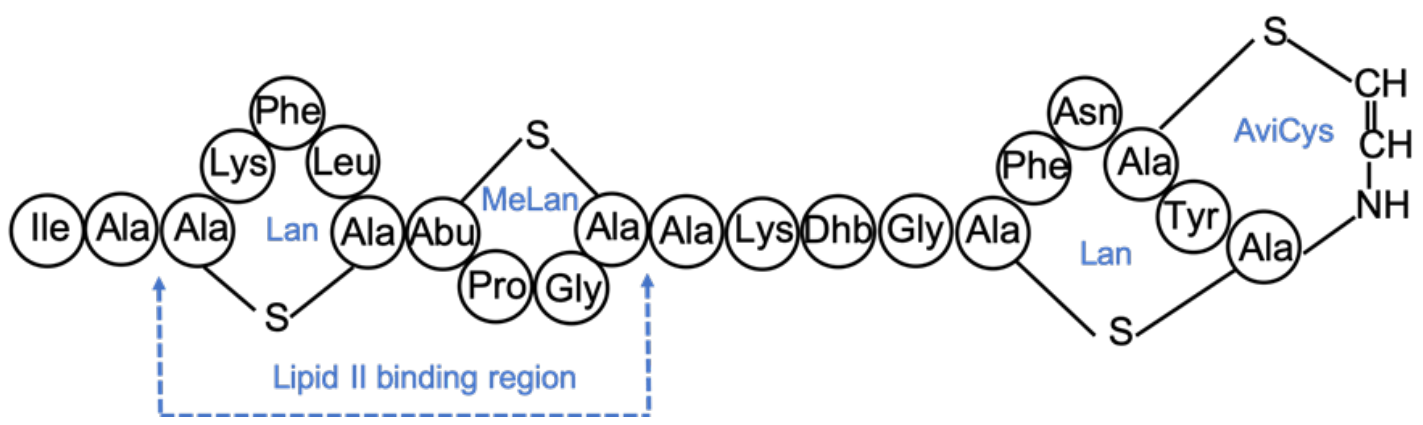

Figure 1.2 The primary sequence of a lantibiotic, Gallidermin. It has four sulfur bridged rings and first two rings serve as a lipid II binding moiety.

Lantibiotics are especially important in current antibiotic research because of their efficacy against resistant Gram-positive bacterial strains that result from unique modes of action. The first two rings of lantibiotics bind to the pyrophosphate of bacterial membrane lipid II molecules and impede the critical functioning of lipid II in bacterial cell wall biosynthesis. Lipid II has two binding moieties for antimicrobial peptides. One is the peptide moiety that is the target for current clinical drugs, including vancomycin, and the other moiety is the pyrophosphate group targeted by several lantibiotics ${ }^{60}$. The last two 
rings of lantibiotics can flip and insert into the bacteria membrane to form membrane pores that disrupt the membrane homeostasis in bacteria ${ }^{61-62}$. The latter mode of action is especially prominent for Class I lantibiotics because they have flexible hinge regions ${ }^{63-65}$. These multiple modes of action to kill bacteria, especially targeting pyrophosphate of lipid II lessens the frequency of development of resistant bacterial strains. Nevertheless, some studies have reported that a few bacterial strains ${ }^{66-67}$ developed some resistance against nisin via modifying their cell wall, forming biofilms, and expressing additional proteins. To date, little is known about the molecular mechanisms or horizontal transfer of lantibiotic resistance in comparison to resistance to current commercial antibiotics ${ }^{44,50,68}$.

Though nisin has been used in food as preservatives for more than 60 years, there are no other lantibiotics in clinical usage. Several lantibiotics, such as MU1140 variants, NAI-107, and NVB302 are currently in pre-clinical and clinical trials ${ }^{69}$. Experimental and computational studies on nisin, MU1140 and gallidermin have provided information on how they form functional membrane pores that can affect membrane permeability, but there is need for atomic-scale investigation that investigates and compares the lipid II binding, and stability of membrane-pores among these variants along with others ${ }^{63,70-72}$. Information on the dynamics of pore formation will be especially valuable when coupled with information on the peptide's ability to interact with lipid II molecules. Such information can be used to guide the design of more efficient lantibiotics with improved anti-pathogenic properties and optimized therapeutic profile. 
1.2 Molecular Dynamics Computer Simulation

In most of my Ph.D. projects, I used molecular dynamics (MD) ${ }^{73}$ computer simulations to investigate conformational changes and recognition of biomolecules. The original implication of MD simulation dates back to the $1960 \mathrm{~s}$, and nowadays it is extensively exhausted to a wide range of applications, including designing novel materials, and drug discovery ${ }^{74}{ }^{75}$. By employing the basic laws of physics, the MD simulation computes the interaction between atoms of biomolecules. The atoms of biomolecules are represented as classical particles, and a potential energy function is used to determine the interaction between these atoms. The potential energy function, generally called force field, contains all possible interactions, as given in equation 1.2.1.

$$
\begin{aligned}
U_{\text {total }} & =\sum_{\text {bonds }} K_{b}\left(r-r_{0}\right)^{2}+\sum_{\text {angles }} K_{\theta}\left(\theta-\theta_{0}\right)^{2}+\sum_{\text {dihedrals }} K_{\varphi}\left[1+\cos \left(n \varphi-\varphi_{0}\right)\right] \\
& +\sum_{\substack{\text { van der Waals } \\
i, j \text { pairs }}}\left(\frac{A_{i j}}{r_{i j}^{12}}-\frac{B_{i j}}{r_{i j}^{6}}\right)+\sum_{\substack{\text { electrostatic } \\
i, j \text { pairs }}} \frac{q_{i} q_{j}}{\varepsilon r_{i j}}
\end{aligned}
$$

The interaction energy is also represented in Figure 1.3. The bonded interactions include bond, angle, and dihedral term. A harmonic potential is applied to model the covalent bond between two adjacent atoms. Similarly, the harmonic angular potential is applied between three adjacent atom pairs. A dihedral term includes four covalently bonded atoms in a linear sequence, where $\varphi$ is the torsional angle of a central bond connected to two covalent bonds. The dihedral interaction is periodic. Other bonded harmonic interactions can include "improper terms" to describe four planar atoms' chirality bound with covalent bonds. The non-bonded interactions comprise Van der Waal's energy defined by Lenard Jones 6-12 potentials and the pairwise electrostatic interaction given by 
Coulomb's Law. A switching function is applied to smoothly cut off the non-bonded interactions at user chosen cutoff distances to reduce the computational time and complexity. In general, the cutoff distance of $12-14 \AA$ is used in MD simulations.

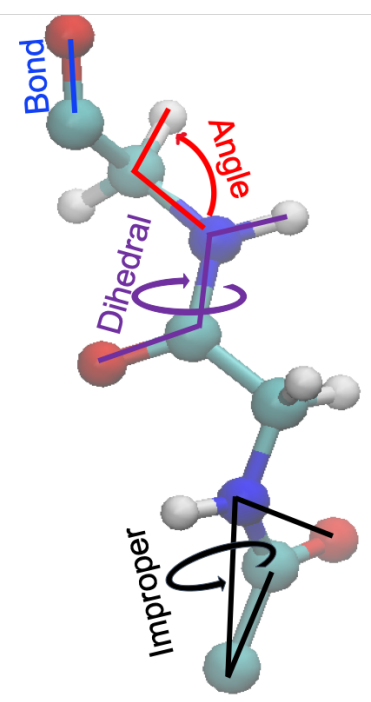

Figure 1. 3 Bonded interactions between atoms of a molecule.

For MD simulations, the starting position of atoms of a biomolecule is assigned from the three-dimensional structure from the x-ray crystallography, NMR, cryo-EM, or homology modeling. The Maxwell Boltzmann distribution of velocities is used to set initial velocities of atoms at a given temperature. The gradient of potential energy function described above gives the force of interaction between atoms and their acceleration. After a user-defined short time step, the position and velocity of atoms are updated. The MD trajectory contains time evolution data of position and velocity of each atom at every time steps. Various methods and tools from statistical physics are used to investigate the biomolecule's thermodynamic property from the MD trajectory. The obtained result can be validated with experimental observables. 
To capture the atomic vibrational events and for the stability of simulation systems, MD time steps are limited to the femtosecond time scale. Hence it is impossible to observe significant conformational changes in biomolecules which usually occurs in a time scale above milliseconds. Though current advancement in Graphics Processing Units (GPUs) has significantly accelerated the MD simulations time, only a few research groups have been able to mark millisecond scale simulation provided with the most advanced computational facilities. It is also nearly impossible for large biological systems to hit that time scale as whole virus capsids or cells. Hence, computational scientists are constantly aspiring for enhanced sampling simulation methods that employ non-equilibrium statistics such as replica exchange MD, Steered MD, Umbrella Sampling, Targeted MD, CoarseGrained MD, etc. The current study used most of these techniques to study protein dynamics, membrane protein interactions, and membrane-pore formation.

\section{METHODS}

\subsection{All Atom Molecular Dynamics (AAMD) Simulation Protocol.}

All-atom MD simulations were performed using the GPU version of NAMD2.12 ${ }^{76}$ with the CHARMM36m force field ${ }^{77}$ employing periodic boundary condition in explicit water solvent. The MD force field parameters for the lantibiotics' unusual amino acids Dha, Dhb, and Abu were taken from previous work on nisin by Turpin et al. ${ }^{78}$ The charge of each system was neutralized by of $\mathrm{NaCl}$ and solvated with TIP3P water model. For EBOV delta peptide membrane pore systems, $0.25 \mathrm{mM}$ concentration of $\mathrm{NaCl}$ was used to increase the ion flux events. For all other simulations it was $0.15 \mathrm{mM}$ to mimic the 
biophysical conditions. The simulation systems without membrane were generated using CHARMM-GUI solution builder while for the membrane systems CHARMM-GUI membrane builder web server was used ${ }^{79}$. For the delta peptide membrane systems, I used asymmetric membrane containing various lipids: POPC, POPE, POPS, POPI, PSM and CHOL with 41:8:4:4:23:20 ratios in the outer leaflet and 11:37:16:10:5:21 ratios in the inner leaflet, which are in accordance with the asymmetric complexity of the plasma membrane $e^{4,80-83}$. The bacterial lipid bilayer for lantibiotics contains POPE and POPG lipids in the ratio of 1:3 to represent the membrane of Gram-positive bacteria ${ }^{84}$.

Systems without membrane were minimization for at least 10,000 steps and equilibrated for 100ps. The minimization and equilibration dynamics of the membrane system were performed following the CHARMM 6-step protocol. Periodic boundary conditions were employed in all simulations. The long-range electrostatic interactions were treated using the particle mesh Ewald (PME) method ${ }^{85}$. The Particle Mesh Ewald (PME) ${ }^{86}$ method was used to calculate the long-range electrostatic interaction and the SHAKE algorithm ${ }^{87}$ was used to constrain hydrogen atoms. A Nose-Hoover Langevinpiston method was used with a piston period of 50 fs and a decay of $25 \mathrm{fs}$ to control the pressure. All simulations were run at $300 \mathrm{~K}$ temperature with Langevin temperature coupling and a friction coefficient of $1 \mathrm{ps}^{-1}$. For calculations of hydrogen bonds, the cutoff distance and cut-off angle were $3.5 \AA$ and $30^{\circ}$ respectively. The visualization tool $\mathrm{VMD}^{88}$ was used to visualize the trajectories and render the pictures. 


\subsection{Steered Molecular Dynamics (SMD)}

The SMD simulation mimics the optical tweezer or atomic force microscopy experiments on shorter time scales ${ }^{89}$. In a molecule, one group of atoms are fixed in a position, and center of mass of other group is pulled in a particular direction. The harmonic constrained with force $\mathrm{k}$ is applied to the center of mass of SMD atoms to move with velocity $\mathrm{v}$ in the direction $\vec{n}$. As a result, the potential applied to the system is:

$$
\mathrm{U}\left(\vec{r}_{1}(\mathrm{t}), \vec{r}_{2}(\mathrm{t}), \ldots, \mathrm{t}\right)=\frac{1}{2} \mathrm{k}\left[\left(\mathrm{vt}-\left(\vec{R}(\mathrm{t})-\vec{R}_{0}\right) \cdot \vec{n}\right]^{2}\right.
$$

$\vec{R}_{0}$ and $\vec{R}(\mathrm{t})$ are the center of mass of SMD atoms at initial and at time $\mathrm{t} . \mathrm{t}=\mathrm{Ns} * \mathrm{dt}$, where Ns is the number of elapsed time steps, and dt is the size of a time step. The variable $n$ with arrow $\left(\overrightarrow{n)}\right.$ is a normalized vector in pulling direction ${ }^{76}$. For SMD, the dummy atom is attached to the COM of SMD atoms via a virtual spring of force constant $\mathrm{k}$.

To break disulfide cross-links between $\mathrm{C} 29$ and C38 and flip the C-terminal anchorage of EBOV delta peptide, I performed SMD simulation with the plasma membrane in explicit water. I pulled the last four heavy atoms of the Arg-39 sidechain along the zaxis at a constant velocity of $1 \AA / \mathrm{ns}$ for $20 \mathrm{~ns}$ with a spring of constant $\mathrm{k}=5 \mathrm{kCal} / \mathrm{mol} /$ $\AA^{2}$. I also performed the SMD simulations for six hybrid peptides along with MU1140 to explore the membrane insertion profile. For six hybrid models, I pulled the three-carbon alpha atoms of amino acids Glu33, Lys34, and Leu35. For wild-type (WT) I pulled the carbon alpha of Tyr20 and Ala21 along the negative z-axis at a constant velocity of 0.5 $\AA / \mathrm{ns}$ for $100 \mathrm{~ns}$. The spring constant was $\mathrm{k}=5 \mathrm{kCal} / \mathrm{mol} / \AA^{2}$. With additional constrained in fixed and SMD atoms, simulations follow the same protocol as explained in section 2.1. 


\subsection{Umbrella Sampling (US)}

Umbrella sampling is an enhanced sampling method used to estimate the relative free energy of different conformational dynamics along the reaction coordinates ${ }^{90}$. It consists of running separate windows of the reaction coordinates parallelly. For each independent window, the system is constrained to sample a narrow phase space along the reaction coordinate to ensure potential energy distribution overlap between adjacent windows. A harmonic or adaptive bias potential is applied to each window ${ }^{91}$. After the simulations, a post-processing integration methods like weighted histogram analysis method (WHAM) ${ }^{92}$, are used to estimate the unbiased free energy by the umbrella integration. The bias potential applied to the system in a window is:

$$
\mathrm{w}_{\mathrm{i}}(\zeta)=\frac{1}{2} K\left(\zeta-\zeta_{i}\right)^{2}
$$

Where $\zeta_{i}$ is reference coordinate point, and $\mathrm{k}$ is force constant.

Then the total energy of the system could be written as:

$$
\mathrm{E}=\mathrm{E}_{\text {unbias }}+\mathrm{W}_{\mathrm{i}}(\zeta)
$$

I used US method to estimate the free energy of flipping the delta peptide anchored tail out of the membrane. From SMD trajectory, I made 20 windows of $1 \AA$ each along the z-axis from $z=-13$ to $z=7 \AA$. Similarly, for membrane insertion energy landscape of hybrid peptides, I took 25 different windows from SMD trajectories of each seven systems from $\mathrm{z}=19 \AA$ to $\mathrm{z}=-29 \AA$ each $2 \AA$ distance apart. For both systems, the reaction coordinate was chosen as the distance between the center-of-mass of the pulled atoms relative to the center-of-mass of the membrane along the z-axis. Each window was then run for $10 \mathrm{~ns}$ of NPT simulation after quick minimization and equilibration. The force constant for harmonic bias potential was $5 \mathrm{kCal} / \mathrm{mol} / \AA^{2}$. 


\section{MEMBRANE PORE FORMATION AND ION SELECTIVITY OF THE EBOLA}

\section{VIRUS DELTA PEPTIDE}

This chapter was published in 2019: R. Pokhrel, E. Pavadai, B.S. Gerstman, P.P.

Chapagain, Membrane pore formation and ion selectivity of the Ebola virus delta peptide, Phys Chem Chem Phys, 21 (2019) 5578-5585.

To understand the mechanism of stable pore formation and ion selectivity of the EBOV delta peptide, I modeled the delta peptide viroporin in different oligomeric states, including tetrameric, pentameric, and hexameric forms. I then performed extensive allatom molecular dynamics (MD) simulations in an explicit membrane environment to optimize these oligomeric structures and investigate the dynamics of their pore-forming mechanisms. I analyzed the pore properties for the different oligomers in the lipid-bilayer, including the energies and arrangements of the oligomers, pore radius, and water and ion permeation through the pores. Our study provides helpful information to better understand the stable viroporin pore-forming mechanism of filoviruses and such structural studies may not only assist in the design and development of antiviral drugs but also have relevance to pore-forming mechanisms of antimicrobial peptides. ${ }^{93}$

\subsection{Construction of the Transmembrane Assembly}

The primary structure of the 40 residue EBOV delta peptide was obtained from $\mathrm{He}$ et al. ${ }^{20}$ (Figure 1.1), and its secondary structure was predicted and modeled using the protein homology/analogy recognition software Phyre ${ }^{94}$ (Fig. S1, Supplementary Information $^{29}$ ). The tetramer, pentamer, and hexamer models of the delta peptide pore were built by placing them symmetrically so that their pore diameters were about $10 \AA$ and 
positively charged residues faced inwards to the pore channel (Fig. 3.1) as suggested by He et al. ${ }^{20}$ and Gallaher et. al. ${ }^{30}$ In addition, Thr-5 and Thr-9 were O-glycosylated and a disulfide bridge was added between C-ter Cys-29 and Cys-38. To understand the role of the disulfide-bond in pore stability and ion flux, I also built pentamer and hexamer pore models without the disulfide bond (reduced pore model). In total, I investigated five different pore models: tetramer, pentamer, hexamer with Cys29-Cys38 disulfide bonds, and pentamer and hexamer pores without the disulfide bond.

Each of the pore models were placed in the lipid bilayer using the CHARMM-GUI membrane builder ${ }^{79}$. The charge of each system was neutralized by $0.25 \mathrm{mM}$ concentration of $\mathrm{NaCl}$ and solvated with TIP3P water model. The asymmetric membrane contains various lipids: POPC, POPE, POPS, POPI, PSM and CHOL with 41:8:4:4:23:20 ratios in the outer leaflet and 11:37:16:10:5:21 ratios in the inner leaflet, which are in accordance with the asymmetric complexity of the plasma membrane $e^{4,80-83}$. For each system, the glycosylated $\mathrm{N}$-ter of the peptides were placed towards the lower, inner leaflet of the plasma membrane bilayer (Figure 3.1).
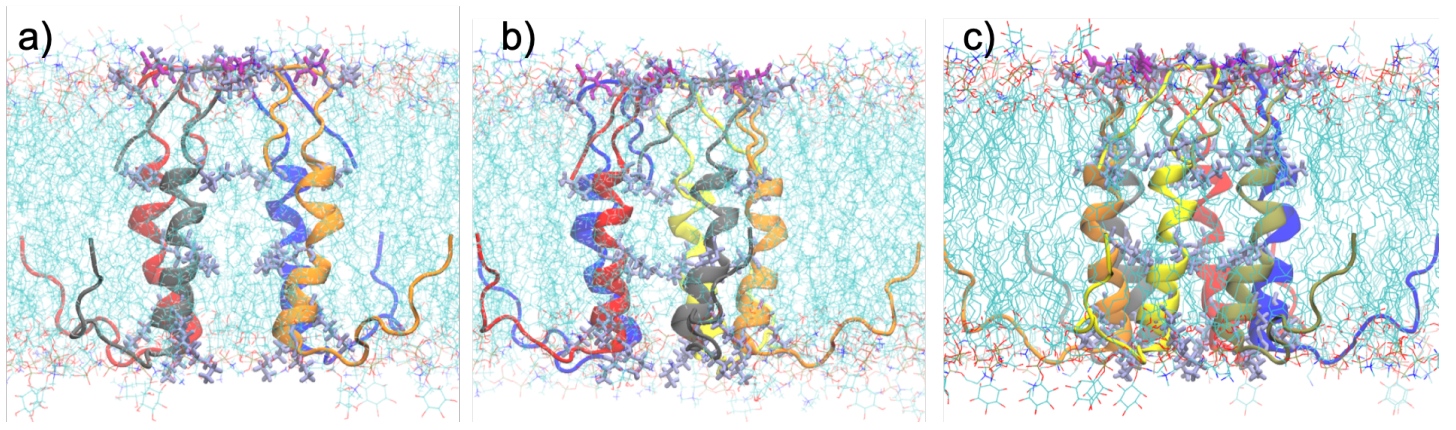

Figure 3.1 The initial structure of the delta peptide pore model for (a) tetramer (b) pentamer (c) hexamer. The $\mathrm{N}$-ter of the peptides are placed at the inner surface of the membrane, which is at the bottom of the diagram. Peptides are shown by ribbon depiction. Color coding: basic residues-blue-gray, acidic residues-purple, membrane-transparent light blue cyan colors. 


\subsection{Delta-Peptide Assembly and Dynamics}

I constructed pores with symmetric placement of four, five, or six delta peptides, according to the orientation of transmembrane amino acids in proposed models from previous studies ${ }^{20,30}$ in the lipid membrane bilayer. The C-ter basic residues (gray-blue at the top in Figure 3.1), Lys-21, Lys-28, Lys-35, and Arg-39 are facing towards the central channel of the pore to provide ion selectivity for chloride ions. In addition, the position of these basic residues in the pores indicates that they can provide a smooth path for chloride ions flux ${ }^{30}$. In contrast, the hydrophobic residues in the middle of the peptide are facing outwards into the hydrophobic middle of the plasma membrane, facilitating pore insertion into the plasma membrane and providing stability to the pore. The Glu-33 residue is at the C-ter, which can hinder the flux of chloride ions from the C-terminal, suggesting that it can act as a gate keeper to regulate ion flux and provide the unidirectional nature of the pores from N-ter to C-ter. The assembly of the pore models suggests that they can pump out water and chloride ions from animal cells, consistent with the EVD symptoms of fever, weakness and multi-organ failure characterized by severe fluid loss and electrolytic imbalance $^{17,20,30,41}$.

To investigate the structural and dynamical properties of the different EBOV delta peptide pore formations, I performed $>500 \mathrm{~ns}$ all-atom, explicit solvent MD simulations in a lipid-bilayer for all three pore structural models that contain the disulfide bond: tetramer, pentamer, and hexamer. The simulated systems and the timescales are listed in Table S1 (Supplementary Information) $^{29}$. I quantitatively analyzed the stability of the pore structures in a variety of ways. I computed the root mean square deviations (RMSD) summed over 
all of the $\mathrm{C} \alpha$ atoms of the pore structures relative to their initial positions (i.e. initial frame of $\mathrm{MD}$ as the reference structure) as a function of simulation time as shown in Figure 3.2a. All pore models reached an apparent equilibrium after $200 \mathrm{~ns}$ with an RMSD compared to their initial structures of approximately $4.5 \AA$. Of the three models, the pentamer model showed more stabilized RMSD values. To track this behavior at longer timescales, I extended the pentamer simulation to $1.1 \mu$ s and plotted the RMSD in Fig. S2

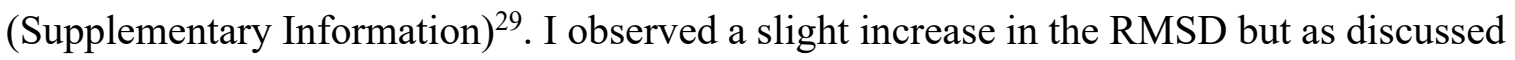
later, the pore structure is further stabilized.

The flexibility of the pore structures was further analyzed by means of the root mean square fluctuation (RMSF) determined from the last $100 \mathrm{~ns}$ of the MD simulation trajectories. The results for the $\mathrm{C} \alpha$ of each residue are displayed in Figure 3.2b. The RMSF shows that the pentamer is the most stable and the tetramer has the largest movement of its $\mathrm{C} \alpha$ atoms, signifying that there is a considerable motion between the peptides in the tetramer. This is in good agreement with the RMSD analysis. The analysis of the RMSD and RMSF shows that the pentamer pore is a more stable viroporin compared to the tetramer and hexamer pores. 

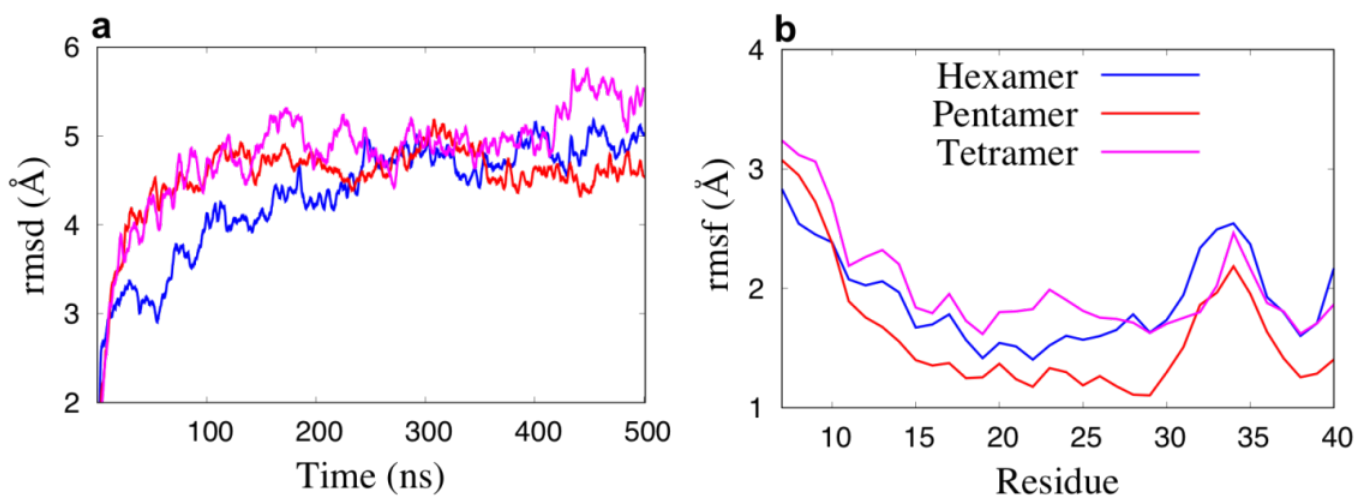

Figure 3.2 For tetrameric, pentameric, and hexameric pores: (a) Time evolution of the root mean square deviation (RMSD) from the initial position averaged over all $\mathrm{C} \alpha$, and (b) root mean square fluctuation (RMSF) in the position of each $\mathrm{C} \alpha$ averaged over the last $100 \mathrm{~ns}$.

To further understand the conformational changes of the peptides, in Figure 3.3 I compare the initial structures of the tetramer, pentamer, and hexamer pores to a snapshot taken at 500 ns. In all cases, the pore structure remains intact but changes in the arrangement of individual peptides can be seen, especially in the tetramer and hexamer. In addition, visual inspection of the initial structure compared with its final structure shows that the tetramer and hexamer pores have lost more of their initial symmetric arrangement and become more disordered compared to the pentamer pore. 

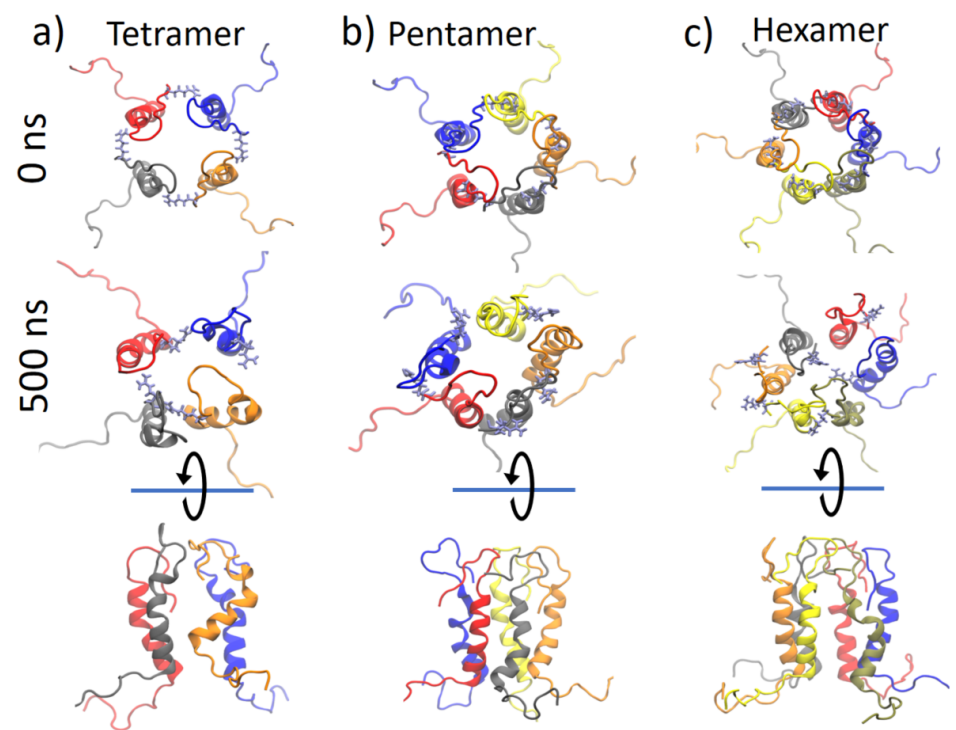

Figure 3.3 Comparison of initial pore structure to pore structure at $500 \mathrm{~ns}$. The top and middle row are end views looking from outside the membrane and the bottom is a side view. Columns: (a) tetramer (b) pentamer and (c) hexamer. In the end views, Arg-39 is shown as gray-blue color.

\subsection{Hydrogen Bond and Interaction Energy Profiles in Various Pore Assemblies}

To further quantify the stability of the different pore models, I analyzed non-bonded interactions: H-bonds, electrostatic, and van der Waals interaction energies, as shown in Figure 3.4. The inter-helical interaction energy was calculated for all peptide pairs for each pore model and then divided by the number of peptides to obtain the average energy. The averaged electrostatic energy is approximately the same for all oligomers after $300 \mathrm{~ns}$ of simulation time. However, the van der Waals energy plot (Figure 3.4b) clearly shows the tetramer has weaker van der Waals interactions than the pentamer and hexamer. Figure 3.4c shows the average number of hydrogen bonds for peptide pairs, normalized in a similar way as for the interaction energy calculations. In the beginning of the simulations, all pore models show a similar trend, but at the end of the simulations, the pentamer has a larger number of hydrogen bonds (three) compared to the tetramer and hexamer (two). The 
results displayed in Figure $3.4 \mathrm{~b}$ and Figure 3.4c indicate that peptide arrangement symmetry and inter-peptide distance play key roles in the stability of the pentamer pore and facilitate the formation of more non-bonded contacts between peptides compared to the tetramer and hexamer pore formations.
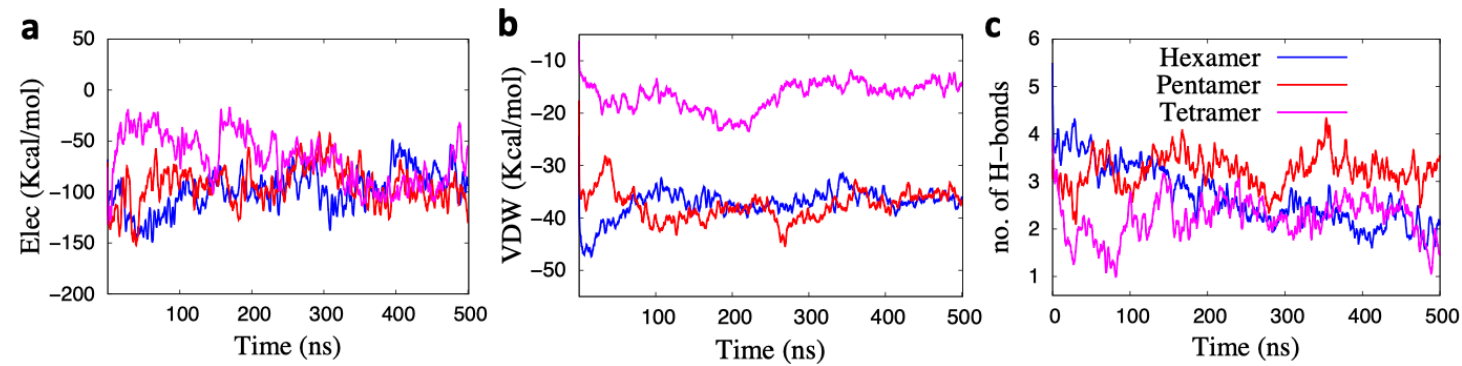

Figure 3.4 Averaged non-bonded pore interactions: (a) electrostatic, (b) van der Waals, and (c) H-bonds.

\subsection{Pore Size, Water and Ion Fluxes}

To gain further insight into delta peptide pore stability, the radius of each pore was averaged over the last $300 \mathrm{~ns}$ of the simulation trajectories using the HOLE program ${ }^{95}$. This program has been extensively used to study the size of protein channels ${ }^{96-98}$ and computes the transverse radius of pores based on the van der Waals radius of the atoms. Figure 3.5a shows the average pore volume, calculated using HOLE. In Figure 3.5b, I display the pore radius along the long z-axis of the channels during the final $300 \mathrm{~ns}$ along with the standard deviation. The mid-point of the membrane is chosen to define $\mathrm{z}=0$. In all three pores, the average radius of the pores is greater than $2.5 \AA$, giving a diameter greater than $5 \AA$, which is significantly larger than the diameter of a water molecule, $2.8 \AA$. Though it is composed of the fewest number of peptides, the tetramer displays a relatively large pore size because of the structural distortion of the peptide arrangement during the 
simulation. The pentamer shows a smaller variation in its average pore size along the length of the channel and smaller standard deviation than the tetramer or hexamer.

In addition, Figure $3.5 \mathrm{~b}$ shows that for all pore profiles, the $\mathrm{N}$-ter mouth of the pore at $z=-18$ has a radius of approximately $4 \AA$, which is wide enough for three water molecules. In contrast, the $\mathrm{C}$-ter mouth of all of the pores at $\mathrm{z}=+18$ have constricted pores of radius $3 \AA$. This difference in the pore size suggests that the pores allow water and ions to flow from N-ter to C-ter. The tetramer and hexamer have greater fluctuations in their pore radius at both ends of the pores than the pentamer, as shown in Figure 3.5 and Movie S1(Supplementary Information) $)^{29}$, showing that the pentamer pore is more stable. In the Movie, sections that are too narrow to pass any water molecules are colored red, green if they allow one water molecule to pass, and blue if they are wide enough to pass two or more water molecules. The $\mathrm{C}$-ter region containing the acidic residue Glu-33 of the hexameric pore contains more frames having red sections than the pentamer and tetramer. The tetramer has wider bulging in the middle region of the pore. In contrast, the pentameric pore remains well formed throughout the simulation. To track the pore stability of the pentamer model at longer timescales, I extended the simulation for this system to $1.1 \mu \mathrm{s}$. Comparison of the pore radius calculated for 300 to 500 ns and 900 to 1100 ns shows a significantly reduced fluctuation, indicating a stabilized pore as shown in Fig. S3 (Supplementary Information) ${ }^{29}$. In addition, Fig. S3 also shows narrowing of the pore radius near the top of the channel. The pore is still able to pass $\mathrm{Cl}^{-}$ions but the narrowing at one end may help in ion selectivity. 


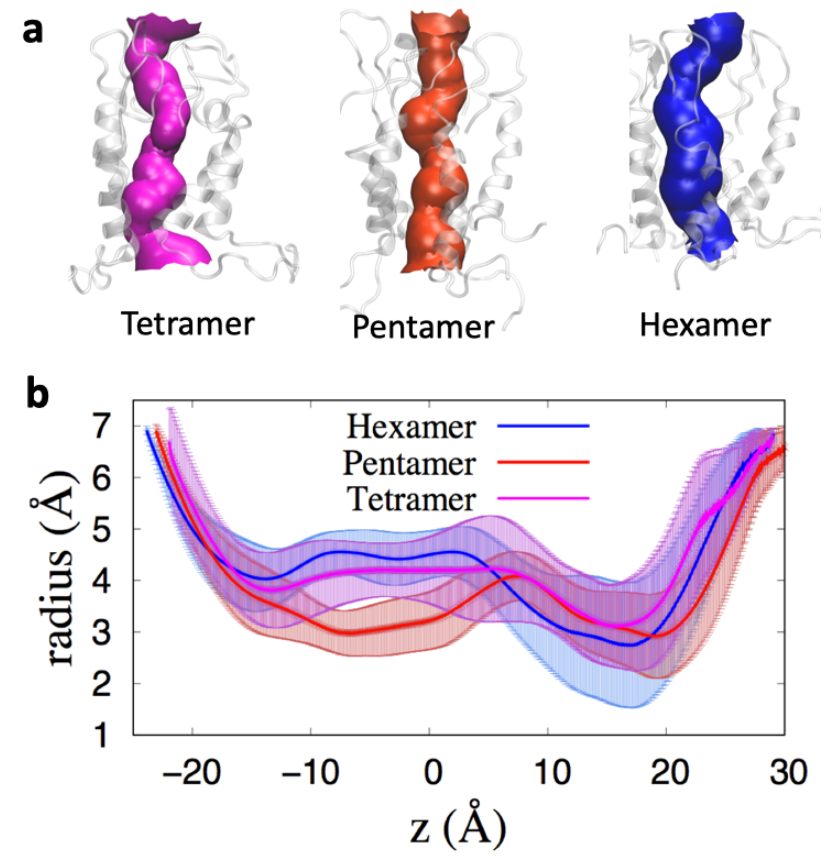

Figure 3.5 a) Volumetric map of the pores for various oligomeric assemblies. b) Average radius of pores calculated from the last $300 \mathrm{~ns}$ of the MD simulations. The volumetric maps and the radius profiles are colored the same way in a) and b).

To explore the pore permeability, the relative density of water, and chloride and sodium ions through the pore were analyzed with the VMD Density Profile Tool ${ }^{99}$. The density is the one-dimensional density calculated along the z-axis of the pores and averaged over the last $300 \mathrm{~ns}$ of the MD trajectories. This analysis shows the presence and absence of water and ions in the pore and is a measure of their permeability. Figure 3.6 shows the relative number densities along the pore, with $\mathrm{z}=0$ at the center of the pore. As shown in Figure 3.6a and Figure 3.6b, all three oligomers are capable of passing water and chloride ions, but in Figure 3.6c the density of sodium ions is zero around the center for all three pores. This result clearly suggests that the pores selectively pass water and chloride ions. The presence of lysine and arginine basic residues in the pores (Figure 1.1 and Figure 3.1) 
facilitate the ion permeation. The chloride ion permeation in the pentameric pore is shown in Movie $\mathrm{S} 2^{29}$.
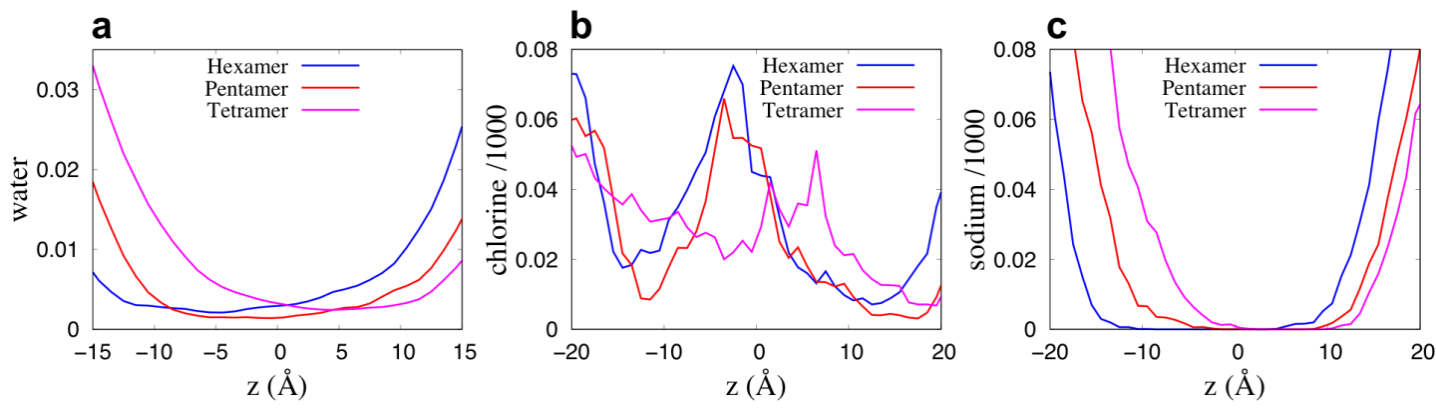

Figure 3.6 Along the length (z-axis) of the pores, the relative density profile of: (a) water (b) chloride (c) sodium

The density profile indicates a significant presence of chloride ions within the pores; however, the density analysis is not a complete measure of the flux of ions through the pores. To understand which of the pores is most effective for chloride ion transport, the passage of chloride ions from the inner leaflet to the outer leaflet (Figure 3.7: OUT) and vice versa (Figure 3.7: IN) are plotted in Figure 3.7. In Figure 3.7, the 500 ns MD simulation is split into 100ns bins. The number of IN and OUT chloride ion flux is plotted for each time bin for each pore. Figure 3.7 shows that the hexamer pore has the largest number of chloride ions traveling through in both directions (35 OUT, 27 IN). This may be due to distortions of the hexamer pore such as reorientation of the basic Arg-39 residue in the C-ter tail. This distortion of the hexamer pore may be destabilizing and at later times lead to the disruption of the pore. In contrast, though the pentameric pore shows a smaller flux of chloride ions (13 OUT, $10 \mathrm{IN})$, the pentamer pore retains its symmetric structure throughout the simulation with little sign of distortion or disruption. The tetramer pore 
displays the least usefulness with a reversed chloride ion flux (5 OUT, 13 IN) and high distortion and destabilizing fluctuations (Figure 3.2 and Figure 3.3).

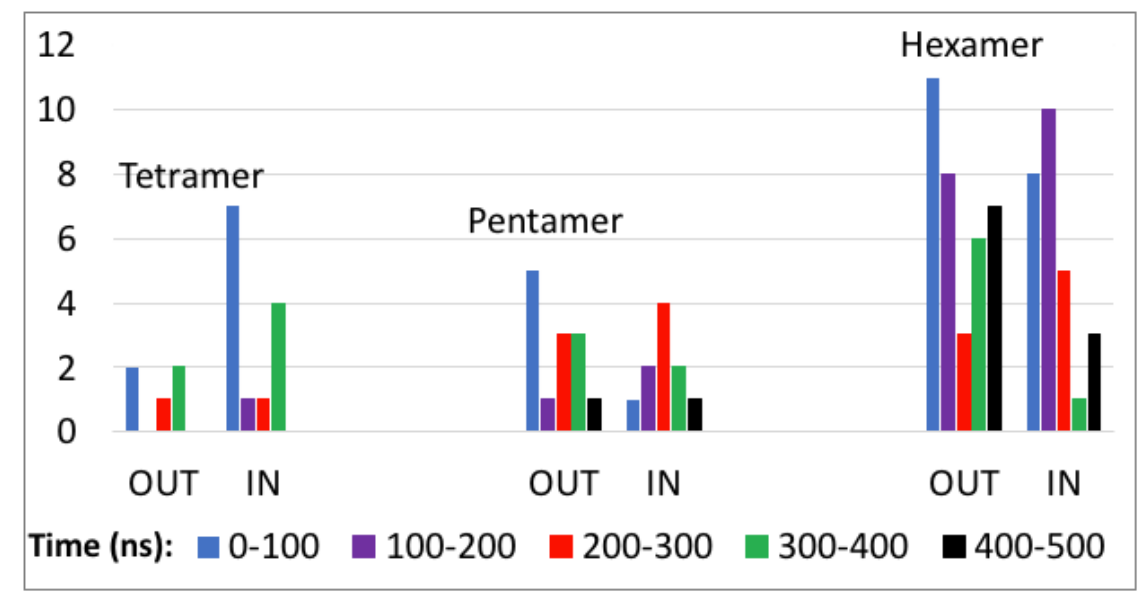

Figure 3.7 Flux of $\mathrm{Cl}^{-}$ions through the pores for (a) tetramer (b) pentamer (c) hexamer. The hexamer allows the largest flux.

\section{5 Role of the Disulfide-Bond in Pore Formation and Ions Flux}

The presence of conserved disulfide-bonds in a wide range of amphiphilic antimicrobial peptides is important for membrane permeation and pore-forming $\operatorname{activities}^{32}$. Recent experimental study demonstrated that the formation of the conserved disulfide-bond between the C-ter Cys-29 and Cys-38 in the EBOV delta peptide is essential for its membrane permeability and pore formation ${ }^{20,41}$. To better understand the role of the conserved disulfide-bond in pore formation and permeability, I performed MD simulations with the pentamer and hexamer pores, but constructed of peptides without the disulfide bond (reduced state of Cys-29 and Cys-38 residues), and compared the results to the pentamer and hexamer pores that have strong peptide disulfide-bonds. I did not study the tetramer pore under the condition of no disulfide bonds since it is the least stable oligomer with the lowest pore forming capability. 
First, I determined if the absence of the disulfide bond in a peptide could cause the C-ter tail (residues 32-40) to flip out of the membrane and into the aqueous environment. For a peptide without the disulfide bond, I performed Steered Molecular Dynamics (SMD) simulations to calculate the relative free energy of the C-ter tail if it remains in the membrane compared to if it is exposed to solvent. The change in position of the tail from inside to outside the membrane bilayer is shown in Figure 3.8a. The free energy curve (Figure 3.8b) for the pulling that flips the C-ter out of the lipid bilayer shows that the configuration of the tail has a lower free energy when it was flipped out of the membrane $(\mathrm{z}>20 \AA)$ and exposed to solvent.
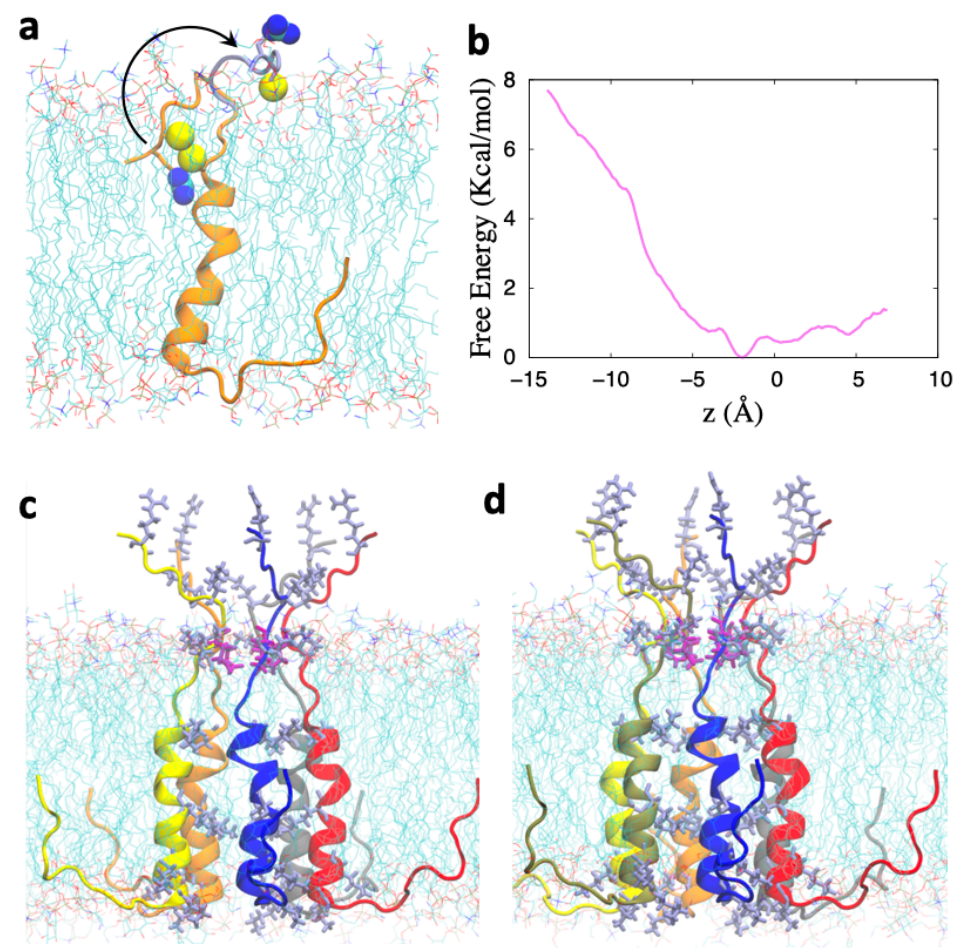

Figure 3.8 For peptides without the disulfide bond in the C-ter region: (a) Initial and final configuration of SMD simulation of a single delta peptide in which the Arg-39 side chain is pulled at constant velocity out of the membrane. (b) Free Energy of the peptide without the disulfide bond as a function of the C-ter tail relative to the surface of the membrane $(\mathrm{z}=0)$ (c) Pentamer pore model, (d) Hexamer pore model, in the bilayer membrane system. 
To determine how pore stability and permeability would be affected in the absence of the peptide disulfide bonds, I performed MD simulations with pentamer and hexamer pore models composed of peptides without disulfide bonds that have their C-ter tail exposed to water (Figure 3.8c and Figure 3.8d) as the initial structure. As shown in Figure 3.9, the pores without the peptide disulfide-bonds show a much larger RMSD compared to the pores with peptide disulfide-bonds, indicating that the presence of peptide disulfidebonds stabilize the pores. In addition, the analysis of the pore structures at different simulation times shows that the pores composed of peptides without disulfide bonds display significantly more structural distortion from their initial symmetric structure than pores with peptides containing disulfide bonds (Figure 3.3), especially for the pentamer.

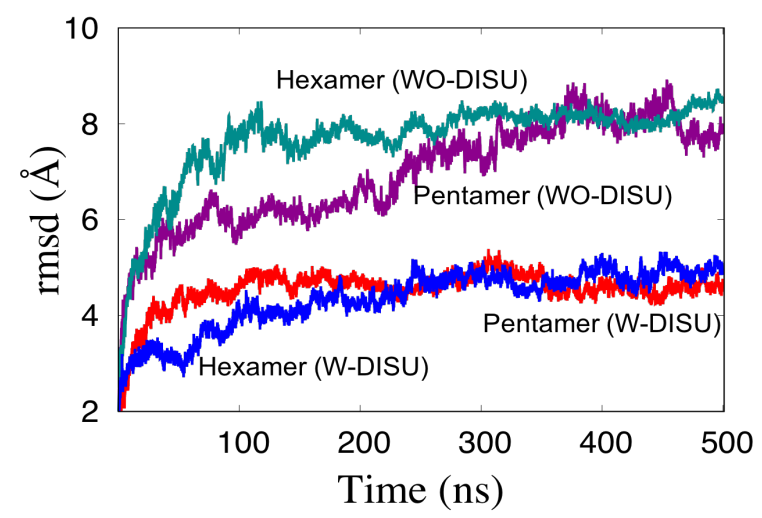

Figure 3.9 For the pentamer pore and the hexamer pore, comparison of the RMSD for the peptides' $\mathrm{C} \alpha$ with and without peptide disulfide bonds. Here, curves for the peptides without the disulfide bond are labeled as WO-DISU and with the disulfide bond are labeled as W-DISU

In addition to the RMSD analysis for the pores without peptide disulfide bonds, the average pore radius, the profiles of chloride ion density and flux of reduced pore models, were also computed and compared to that of pores with peptide disulfide bonds models. As shown in Figure 3.10, the pore-radius in the central region has decreased for the pores 
without disulfide bonds in both pentamer and hexamer models. The pentamer pore without disulfide bonds is still stable but the hexamer pore is significantly distorted in the absence of disulfide bonds, as reflected by the deviation in the pore radius in Figure 3.10b. The much wider fluctuations of the end residues of the pores without peptide disulfide bonds reflect the decreased stability of the pore. This is further supported by a reduction in chloride ion density and flux for the pores without peptide disulfide bonds in Figure 3.10. Overall, the results show that the peptide disulfide-bond is essential to maintaining the structural integrity of the pore and the flux of chloride ions, which is consistent with the experimental suggestions ${ }^{20,41}$.
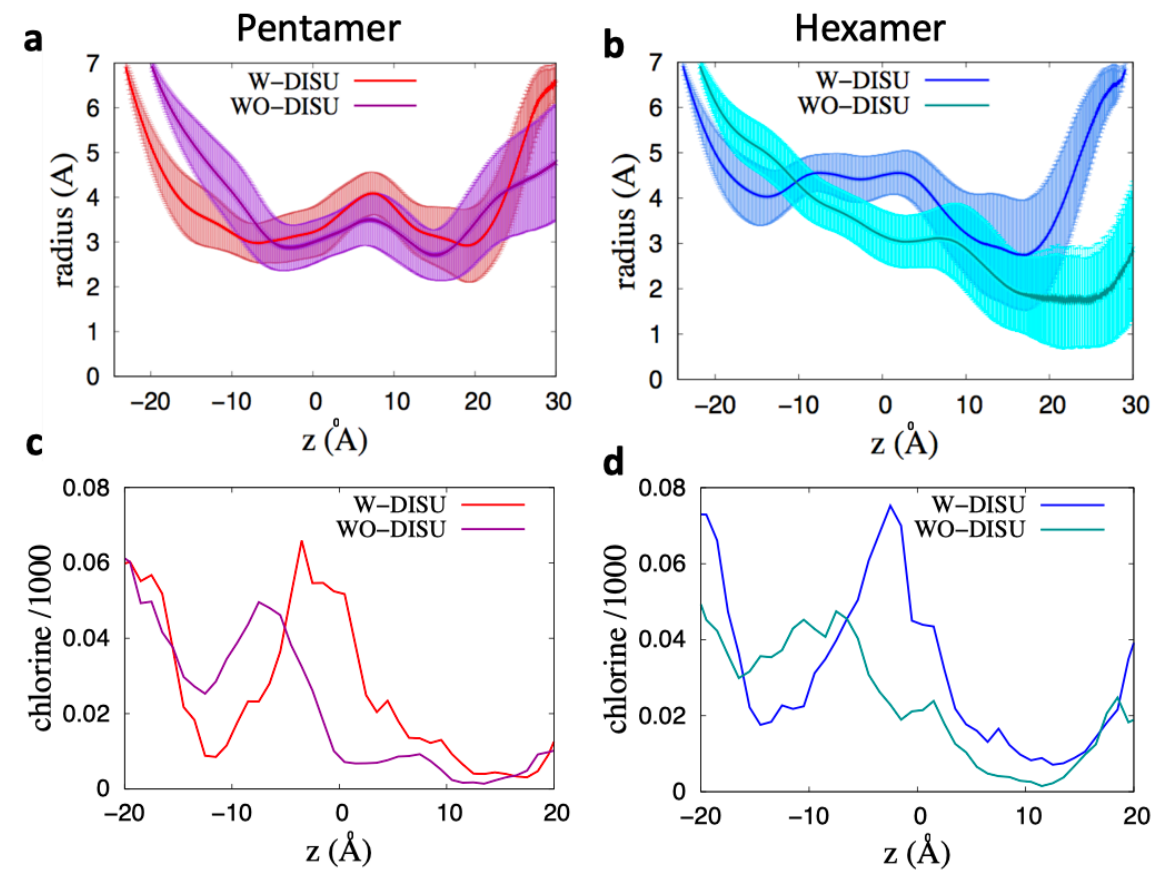

Figure 3.10 a) Average pore radius from the last $300 \mathrm{~ns}$ of the MD simulation for the pentamer and (b) hexamer, composed of peptides with and without disulfide bonds. The shaded region shows the standard deviation of pore radius. The much wider fluctuations of the end residues at the $\mathrm{C}$-ter $(\mathrm{z}>20 \AA)$ of the pores without peptide disulfide bonds reflects the decreased stability of the pore. c) Comparison of average density of chloride atoms along the pore for pores with and without peptide disulfide bonds for pentamer and (d) hexamer. For both the pentamer and the hexamer, fewer chloride ions are found in the pores without peptide disulfide bonds. 
4. LIPID II BINDING AND TRANSMEMBRANE PROPERTIES OF ANTIMICROBIAL LANTIBIOTICS.

4.1 Molecular Mechanisms of Pore Formation and Membrane Disruption by the Antimicrobial Lantibiotic Peptide Mutacin 1140

This chapter was published in 2020: R. Pokhrel, N. Bhattarai, P. Baral, B.S. Gerstman, J.H. Park, M. Handfield, P.P. Chapagain, Molecular mechanisms of pore formation and membrane disruption by the antimicrobial lantibiotic peptide Mutacin 1140, Phys Chem Chem Phys, 21 (2019) 12530-12539.

In this work, I explored the potential mechanism of membrane pore formation by performing molecular dynamics (MD) simulations of the MU1140-lipid II complex in the bacterial membrane. Despite significant structural/sequence differences between the nisin and MU1140 C-terminal tails, our results show that MU1140-lipid II complexes are able to form functional, water permeating membrane pores. These investigations, for the first time, provide an atomistic level insight into a novel mode of action for MU1140 that can be exploited to develop optimized peptide variants with improved antimicrobial properties and therapeutic profile.

\subsubsection{Structural Comparison of MU1140 and Nisin}

The NMR structure of the nisin-lipid II complex (PDB ID 1wco) was used as a template to model the MU1140-lipid II complex (Figure 4.1), preserving the structure of the pyrophosphate binding cage and the lantibiotic-lipid II interactions. With the target being the pyrophosphate moiety, a dipeptide (Ala, Glu) was used for the peptide moiety 
and a longer bactoprenol tail was used that provided a stable anchorage during membranelipid II simulation. As with other lantibiotics chains, MU1140 contains unsaturated amino acid residues dehydroalanine (Dha), dehydrobutyrine (Dhb), Aminobutyric acid (Abu), and a $C$-terminal aminovinyl- $D$ - cysteine (AviCys). It contains thioether rings connecting two amino acids by a sulfur bridge, with Ala-S-Ala as the lanthionine derivative (Lan) and AbuS-Ala as the 3-methyl-lanthionine (MeLan) derivative. Conserved disulfide-bonds are found in a wide range of amphiphilic antimicrobial peptides and viroporins, and are important for membrane insertion and their pore-forming ${ }^{29,}$ 100-101. In Figure 4.1, I compare the sequence and structure of the nisin and MU1140 chains. Compared to the 34-residue nisin, MU1140 is shorter, with only 21 amino acids. Nisin contains five thioether rings, whereas MU1140 contains three thioether rings and a fourth ring formed by heteroatoms at the $C$-terminal tail (Figure $4.1 \mathrm{a}, \mathrm{b}$ ).

Nisin has three Lys residues giving a net +3 charge (excluding $N$-terminus group). In contrast, MU1140 contains a net +2 charge due to two basic amino acids Lys and Arg. The anionic pyrophosphate moiety of lipid II with a net -2 charge provides the binding target for the cationic MU1140. The hydrophilic head group of lipid II consists of two sugar linked rings $\mathrm{N}$-acetylglucosamine (GlcNAc), N-acetylmuramic acid (MurNAc), and pentapeptides connected to MurNAc ${ }^{60,102-103}$. The hydrophobic tail, bactoprenol is linked to MurNac via pyrophosphate moiety. The lipid II binding thioether rings in nisin and MU1140 (positions 3-11) are nearly identical, with only one amino acid difference (Ile vs. Trp in position 4). Therefore, I used the structure of the nisin-lipid II complex (PDB ID 1wco) as a template for modeling the MU1140-lipid II complex, preserving the structure of the pyrophosphate binding cage and the lantibiotic-lipid II interactions. The NMR 
structure of the nisin-lipid $\mathrm{II}^{61}$ complex is shown in Figure 4.1c and our model for the MU1140-lipid II complex (energy minimized and equilibrated structure) is shown in Figure 4.1d.
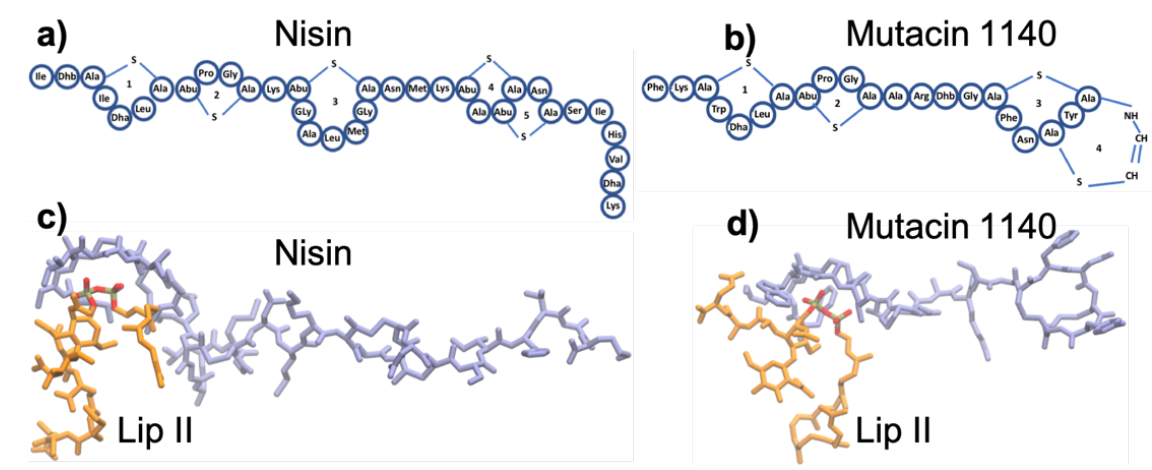

Figure 4.1 Schematic representation of amino acid sequences of (a) nisin and (b) MU1140 chains. The NMR structure of (c) the nisin-lipid II complex ${ }^{61}$ and d) the modeled structure of the MU1140-lipid II complex. The lantibiotic chains are shown in blue and lipid-II in orange. The phosphate and oxygen atoms in the pyrophosphate moiety are highlighted in gray and red respectively.

\subsubsection{MU1140 Interactions with The Membrane Surface and Lipid II}

To investigate the interactions of MU1140 chain with the membrane surface when it is bound with lipid II in the membrane, I placed the MU1140-lipid II complex in the membrane in such a way that the MU1140 chain lies horizontally on the outer surface of the lipid bilayer, and the lipid II is inserted into the membrane, aligned parallel with other lipids in the lipid bilayer (Figure 4.2a). The MU1140-membrane surface interactions are relevant not only for its lipid II trafficking mechanism but also for the initial phase of the peptide insertion into the membrane. I performed a 500-ns simulation of the complex in the bacterial membrane composed of POPE and POPG in the ratio of 1:3.

I observed that the MU1140-lipid II complex remained intact throughout the simulation, with strong interactions between MU1140 and the pyrophosphate moiety of 
lipid II. At the end of the 500-ns simulation, residues in the third thioether ring as well as the $C$-terminal segment show partial insertion into the membrane bilayer (Figure 4.2a). In Figure 4.2b, I plot the percentage of time during the MD simulation that major amino acids in MU1140 form hydrogen bonds with the membrane as well as with lipid II (inset).

The MU1140 cationic side chain of Lys2 makes the major salt-bridge interaction and the backbone NH groups of Phe1 and Lys2 provide strong hydrogen bonding with the pyrophosphate group in lipid II (Figure 4.2b, right). As displayed in Figure 4.2c, MU1140 makes approximately three salt-bridge or hydrogen bonds interactions with lipid II throughout the simulation. In addition, the bar graph for the MU1140-membrane hydrogen bond in Figure 4.2b shows that MU1140 is able to strongly interact with the bacterial membrane surface through significant hydrogen bonding. The major MU1140-membrane hydrogen bond contribution comes from the MU1140 Arg13, followed by hetero-group (AviCys) at the $C$-terminal tail and the backbone interactions of Tyr20. Overall, MU1140 makes about 12 hydrogen bonds on average with the membrane, as shown in Figure 4.2c. In Figure 4.2d, I plotted the number of MU1140 contacts with lipid II within $3.5 \AA$. MU1140 has a nearly constant number of interactions with POPE throughout the simulation, whereas interactions with POPG shows slight increase after $200 \mathrm{~ns}$.

As a control, I performed a separate simulation for the MU1140 chain on the membrane surface while it is not complexed with lipid II. As shown in Fig. S1 (Supplementary Information $)^{72}$, the MU1140 chain interacts with the membrane surface but the partial insertion of the hydrophobic groups is not as pronounced as in MU1140 complexed with lipid II (Figure 4.2a). This suggests that the lipid II interactions in the 
pyrophosphate moiety allows the MU1140 chain to stabilize on the membrane surface and facilitate membrane insertion.
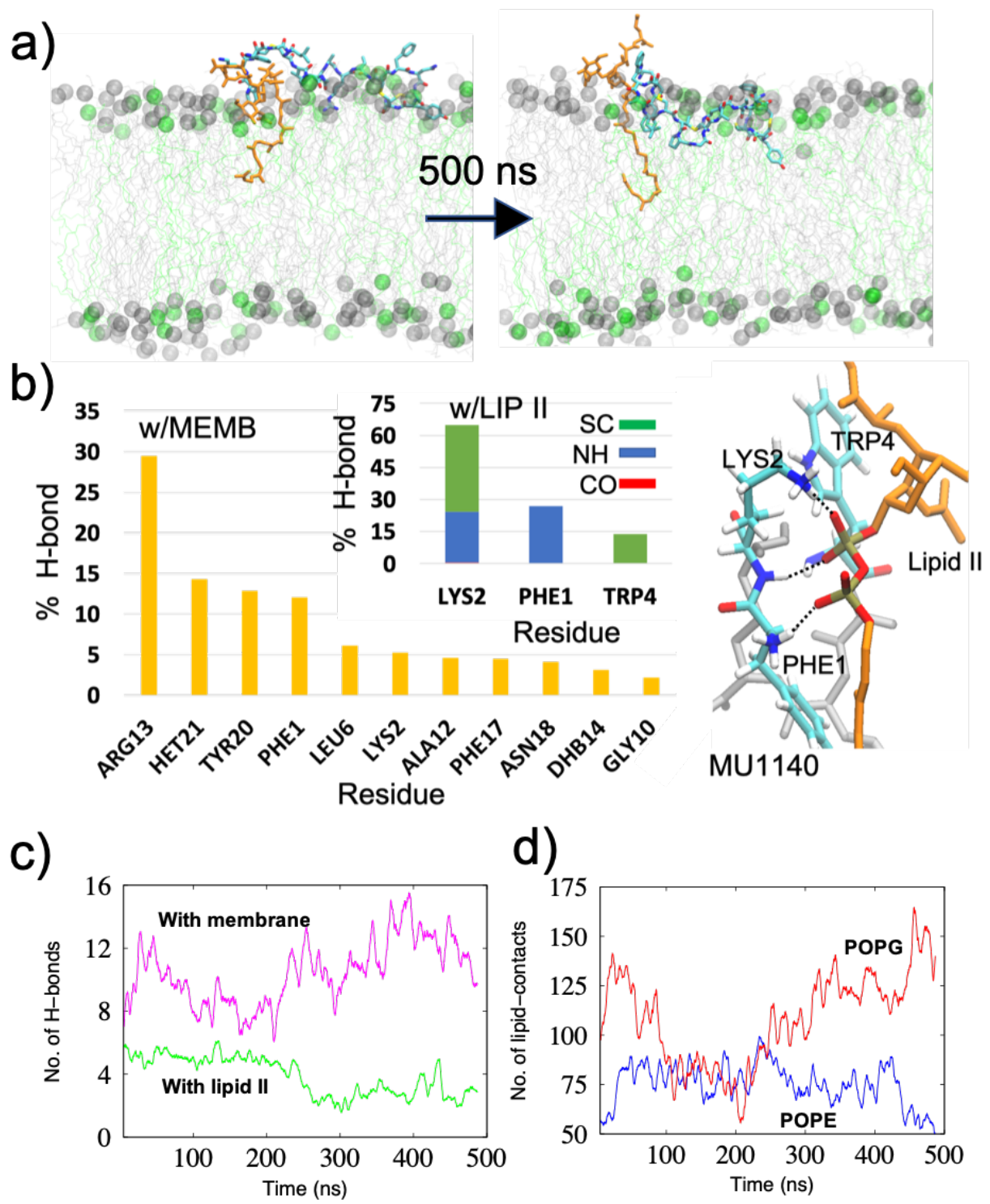

Figure 4.2 (a) MU1140-lipid II complex in the membrane at 0 ns and 500 ns. MU1140 is colored in by atom names, lipid II in orange, POPE in green, and POPG in gray. The phosphorous atoms in the lipid headgroups are shown as spheres. (b) Percentage hydrogen bonds between MU1140 and membrane as well as between MU1140 and lipid II (inset). The \% H-bond between MU1140 and lipid II are colored as, green: sidechain (SC), blue: backbone NH group, and red: backbone CO group. Important MU1140 residues participating in hydrogen bonding with lipid II are highlighted (right). c) Number of hydrogen bonds of MU1140 with lipid II as well as with other lipids in the membrane as a function of time. d) Number of lipid contacts with MU1140 as a function of time. 


\subsubsection{Membrane Insertion of MU1140}

A net cationic charge and a high fraction of the hydrophobic residues are two common features of antimicrobial peptides (AMPs) ${ }^{104}$ and the hydrophobicity can allow the peptide chain to insert into the membrane. MU1140's close analogs, nisin and gallidermin are both shown to form membrane pores ${ }^{105-106,107}$. Based on the similarities of lipid II binding and the peptide composition with respect to nisin, epidermin, and gallidermin, I conjectured that MU1140 can also form functional membrane pores. The observation of peptide insertion in an all-atom computational model is difficult due to temporal limitations of unbiased MD simulations. A 500-ns of detailed all-atom simulation with full-length lipids allowed only a partial insertion of MU1140. To facilitate the membrane insertion in reasonable computational timescales, I used a highly mobile membrane-mimetic (HMMM) model ${ }^{108}$. The HMMM membrane model uses lipids with shortened tails, floating on a layer of 1,1-dichloroethane (DCLE) representing the hydrophobic inner core of the lipid bilayer. The fluidity of the system allows significantly enhanced lipid diffusion that can provide unbiased lipid-binding of the protein well under a microsecond. With the MU1140 chain aligned along the membrane surface (Figure 4.3a) and the lipid II aligned parallel to the lipids, I performed a production run for $1.3 \mu \mathrm{s}$. Figure 4.3a shows the configuration of the complex in the beginning of the simulation (left panel) and after $1 \mu \mathrm{s}$ of simulation (right panel). Figure $4.3 \mathrm{~b}$ shows the vertical distance (z-axis) between Tyr20 and the center-of-mass of the lipid bilayer (representing the lipid bilayer's mid-point). After about $800 \mathrm{~ns}$, the MU1140 chain begins to insert into the membrane and by around $1 \mu \mathrm{s}$, most of the chain that is not interacting with the lipid II pyrophosphate 
cage is in the transmembrane region, as can be seen in Figure 4.3a (right) and the decreasing distance from the lipid bilayer's mid-point in Figure 4.3b. These results show that the MU1140 chain prefers the hydrophobic transmembrane region compared to the outer surface of the membrane.

a)

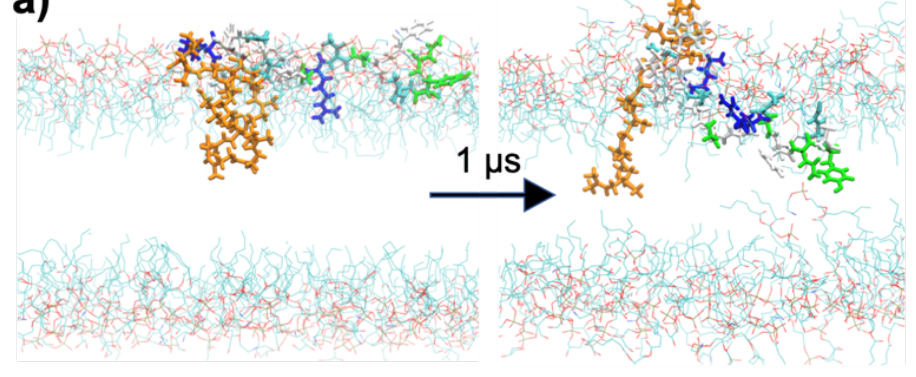

b)

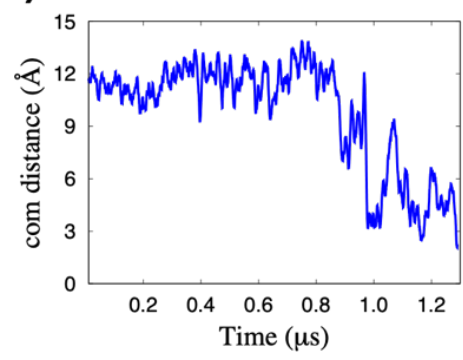

Figure 4.3 a) Insertion of MU1140 into the HMMM membrane. For clarity, DCLE molecules in the transmembrane region are not shown. b) Distance between the Tyr20 of MU1140 and the center-of-mass (com) of the lipid bilayer (membrane mid-point).

\subsubsection{Membrane Pore Formation and Water Permeation by MU1140}

After confirming that the hydrophobic transmembrane region is a favorable environment for the MU1140 chain, I investigated its pore forming ability. While insertion is greatly facilitated by the HMMM membrane, the inserted MU1140 chain is indifferent towards aligning in any specific orientation because of the fluidity of the transmembrane DCLE that does not restrict the peptide chain to a vertical orientation as the full-length lipids would. Therefore, investigation of the pore formation requires a bilayer with fulllength lipids. One way to set this up is to wait for a random even that vertically orients the MU1140 chain in the HMMM membrane and then add the lipid tails. To expedite this process, I set-up MU1140-lipid II complex in a conventional lipid bilayer with full-length lipids but with the MU1140 already inserted and aligned vertically in the transmembrane 
region. I note that use of enhanced sampling methods such as accelerated molecular dynamics may allow investigations of membrane insertion without having to reset the system.

Figure 4.4a shows the initial configuration of the MU1140-lipid II complex in the bacterial membrane composed of POPE and POPG in the 1:3 ratio. I performed a 500-ns simulation for the system and observed a significant water permeation even with a single complex of MU1140 and lipid II. Figure 4.4b shows a representative frame at the end of the 500-ns simulation which shows water molecules along the MU1140 chain in the transmembrane region.

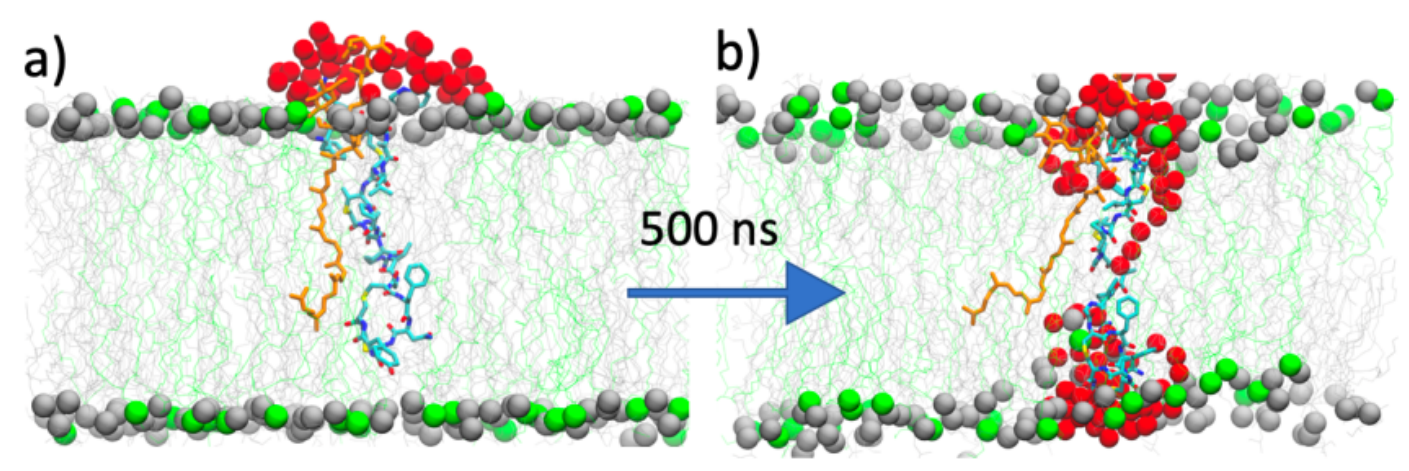

Figure 4.4 MU1140-lipid II complex in a bacterial membrane: a) Initial configuration of the complex and b) a configuration at the end of 500-ns simulation. The phosphorus atoms of POPE are highlighted as green spheres and that of POPG as gray sphere. The water molecules within $4 \AA$ of the peptide are highlighted as red spheres.

Before the simulation began, only the $N$-terminal part of the peptide is exposed to water on the outside of the bacterial membrane and the $C$-terminal is buried in the transmembrane region as shown in Figure 4.4. Within a 100-ns, the lower end of MU1140 inside the membrane is in contact with water molecules below the membrane (intracellular space). Despite having a significantly shorter chain compared to nisin, MU1140 is able to span the transmembrane region and create a channel for water permeation. As with 
gallidermin that also harbors a shorter chain, the water permeation and pore formation by MU1140 likely correlates with the membrane thickness ${ }^{107}$. The bactoprenol tail of lipid II has no particular preference in its orientation and is quite flexible in the transmembrane region. Although lipid II interactions may facilitate membrane insertion of MU1140, simulations of MU1140 chain (not complexed with lipid II) in the transmembrane region showed similar water permeation behavior (Fig. S2) ${ }^{72}$.

\subsubsection{Single-File Water Permeation}

To explore the mechanism of water permeation, I examined the residues and the groups involved in interactions with water. I created a list of all water molecules that come within $3.5 \AA$ of peptide residues and calculated the number of hydrogen bonds between the peptide and water. Figure 4.5 displays a snapshot of a typical frame that shows a singlefile water permeation between the outer and inner layers of the membrane. Movie S1 (Supplementary Information) $)^{72}$ shows how water molecules permeate across the bilayer via hydrogen bonding with MU1140 residues. Single-filed hydrogen bonded chains of water are often observed in nanoscale confinements such as in carbon nanotubes and protein channels (e.g., the aquaporin gramicidin) ${ }^{109-110}$ and are of great interest because of their unusual properties. As shown in Figure 4.5, water molecules in the single-file chain region are considerably ordered, similar to the behavior observed in other biological water channels such as gramicidin $\mathrm{A}^{111-112}$. The importance of the single-file water is underscored by its role in transporting ions. In gramicidin A, the ability of single-file water to stabilize $\mathrm{K}^{+}$ions and transport it through the channel is due to the solvation of the ion by two single file chains on either side ${ }^{113-114}$. Unlike fully non-polar carbon nanotubes, biological water pores are lined with backbone carbonyl and amide groups that can form hydrogen bonds 
with water molecules. As here, water molecules in the single-file region predominantly hydrogen-bond with carbonyl groups of MU1140 residues. With larger assembly of MU1140-lipid II complexes, transport of cations may be possible because the polar lining by the backbone carbonyl groups in MU1140 may form hydrogen bonding with monovalent cations and facilitate ion transport as shown for epidermin and gramicidin ${ }^{115}$.
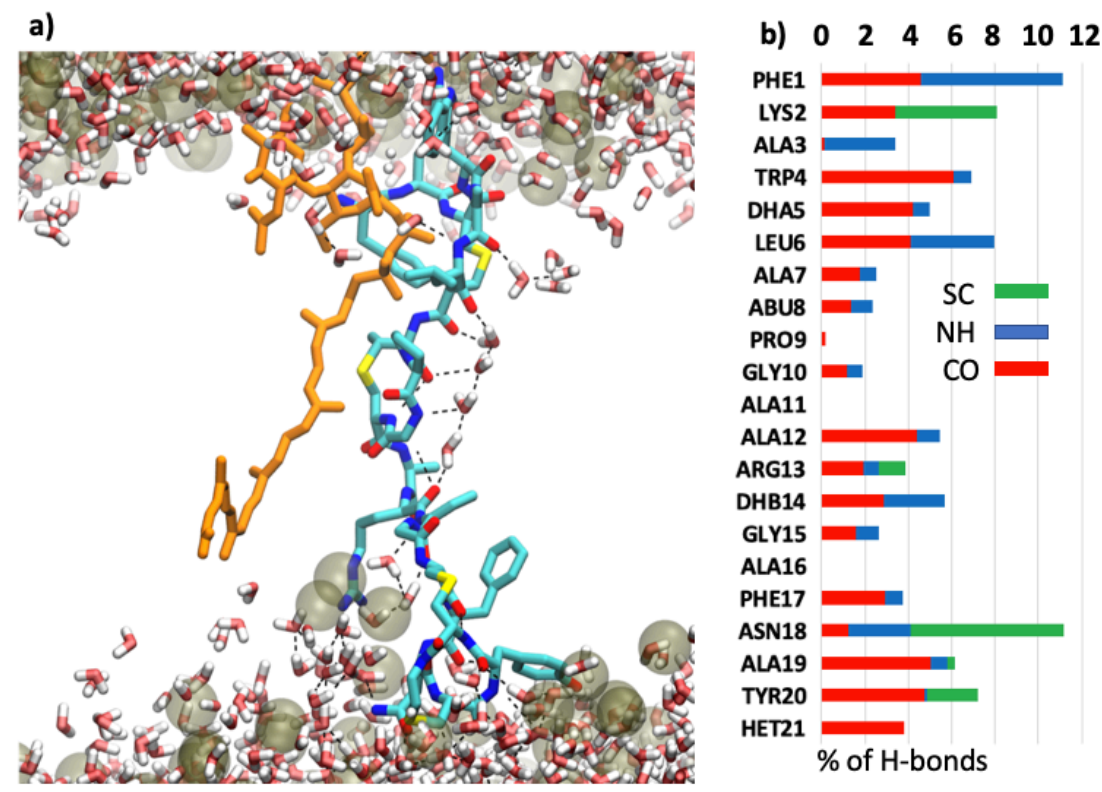

Figure 4.5 a) A representative snapshot of the water permeation along the MU1140 chain (cyan) in the transmembrane region. Lipid II is shown in orange. b) Percentage of hydrogen-bonding between MU1140 residues and water molecules, showing MU1140 hydrogen bonds with backbone $\mathrm{CO}$ (red), backbone $\mathrm{NH}$ (blue), and sidechain (SC) (green). Only the phosphate atoms of the lipids are displayed for clarity.

The MU1140 structure inside the membrane shows a concaving at and below the second thioether ring and this allows a proper orientation of CO and NH groups of MU1140 residues so that the water molecules can line along the chain (Figure 4.5a). I calculated the $\%$ hydrogen bond between MU1140 groups and water molecules and plotted the results in Figure 4.5b. Three residues Pro9, Ala11, and Ala16 are oriented away and make almost no contacts with water. Since Phe1 and Asn18 are close to the bulk water, they are found to 
form most hydrogen bonds with water. While only the backbone groups of Phe1 are involved in hydrogen bonding, Asn18 sidechain makes significant hydrogen bonding with water. It is found that substitution of Phe1 with other resides does not negatively impact MU1140 activity ${ }^{116,117}$ possibly because backbone hydrogen bonding is unaffected. Based on the hydrogen bonding pattern, three different transmembrane regions can be observed along the chain: 1) residues $1-5,2$ ) residues $6-13$, and 3) residues 13-18. Along the sequence Leu6 to Arg13, considerably ordered single-file water is observed. The MU1140 hydrogen-bonding with the single-file water is found to be with Leu6 (CO), Ala7 (CO), Abu8 (CO), Gly10 (NH), and Arg13 (CO). Due to irregular ordering of backbone carbonyl and amide groups, water passage below Ala12/Arg13 to the bulk occurs in a less ordered fashion and can involve a sequence of as many as four water molecules hydrogen bonded between Ala12 and Phe17. Due to the proximity to the bulk water, the Phe1 to Dhb5 as well as Asn18 to HET21 have the water that is less ordered.
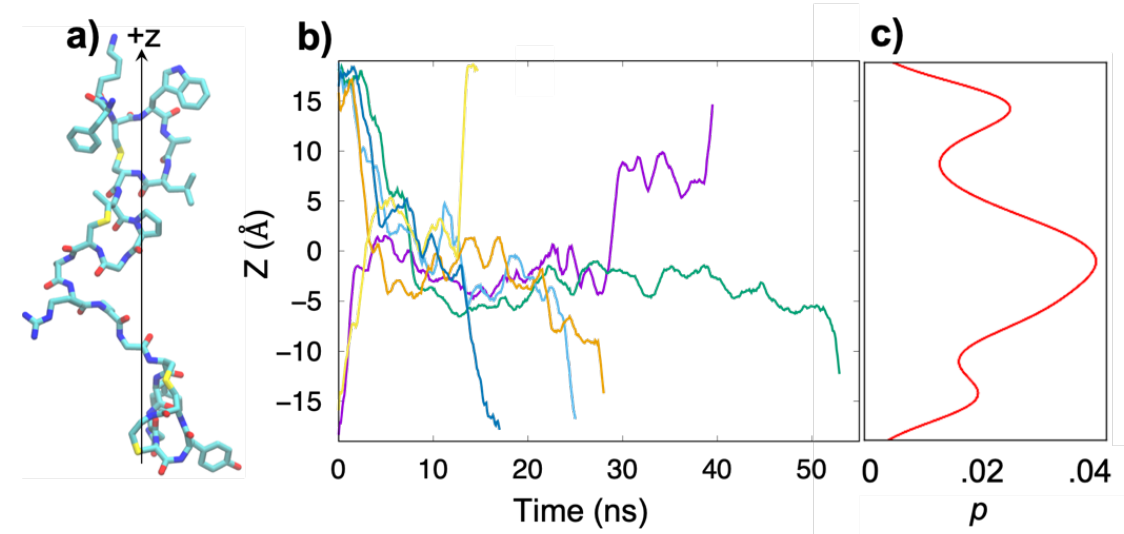

Figure 4.6 a) MU1140 orientation in the lipid bilayer along z-axis b) Representative trajectories of successful water permeation across the membrane. c) A histogram of the number of water molecules along $\mathrm{z}$-axis is shown on the side. 
Significant water ordering and hydrogen bonding around the mid-region of the lipid bilayer causes a longer residence times for the water molecules, with major bottlenecks for the ion passage at Leu6 and Arg13. In Figure 4.6, I plotted the trajectory for a few representative water molecules that successfully permeated along the z-axis across the lipid bilayer. Water transport is observed in both directions. The concavity of the MU1140 in the mid-region allows relatively more extensive hydrogen bonding for the water molecules. I also plotted the histogram from all the successful trajectories (right panel), which shows a significantly populated mid-region (left panel). This is consistent with the observations in other biological systems which show markedly reduced diffusion of water with the increase in hydrogen bonds with the pore-lining residues ${ }^{117}$. When the single-file water molecules hydrogen bonds with the pore-lining residues, water movement is limited due to the time it takes to not only break the hydrogen bonds but also to reorient and form new hydrogen bonds while moving through the channel. In fact, Horner et al. ${ }^{117}$ found that the flow of single-file water depends more on the number of hydrogen bonds with pore-lining residues than the channel geometry. In contrast, the hydrophobic walls of carbon nanotubes can allow unrestricted water flow ${ }^{109-110}$. In addition to the role of polar groups lining the pore wall, water permeation across the membrane is also limited by the dehydration penalty at the pore entrance. The fairly low population of water at the two extremes (i.e. $|z|>15 \AA$ in Figure 4.6) is likely due to this penalty. The two least populated regions (before Leu6 and after Arg13) represent the transition states for the water passage.

From the successful water permeation trajectories, I extracted the first passage times for the water molecules to fully traverse the lipid bilayer. The mean first passage time (MFPT) for the water molecule was found to be $\sim 23 \mathrm{~ns}$, giving a diffusion constant 
of $\mathrm{D} \approx 0.03 \mathrm{~nm}^{2} / \mathrm{ns}$ (i.e. $3 \times 10^{-7} \mathrm{~cm}^{2} / \mathrm{s}$ ) which is two orders of magnitude slower than the water diffusion in nonpolar carbon nanotubes with $\mathrm{D} \approx 3 \times 10^{-5} \mathrm{~cm}^{2} / \mathrm{s} .{ }^{110}, 118$. Here, the diffusion constant was calculated ${ }^{118}$ using $D=L^{2} / 12\langle t\rangle$, where $L=2.8 \mathrm{~nm}$ is the channel length and $\langle t\rangle=23 \mathrm{~ns}$ is the first passage time. The diffusion constant is similar to the values determined for other biological single file water channels such as aquaporin-1 $\left(\mathrm{D} \approx 4 \times 10^{-}\right.$ $\left.{ }^{7} \mathrm{~cm}^{2} / \mathrm{s}\right)^{117}$, and gramicidin $\left(\mathrm{D} \approx 3 \times 10^{-7} \mathrm{~cm}^{2} / \mathrm{s}\right)^{119}$.

\subsubsection{Water Permeation in Multimeric Assembly of MU1140-Lipid II Complexes}

In many membrane binding and pore-forming amphipathic peptides, pore formation occurs only if the peptide concentration exceeds certain threshold values ${ }^{120}$. In a physiological environment, multiple MU1140 and lipid II molecules are likely to assemble in the membrane. While nisin has 1:1 binding stoichiometry in solution ${ }^{121}$, it is suggested to form membrane pores with 2:1 ratio of nisin-lipid II assembly ${ }^{64}$. However, there is no information available about MU1140-lipid II assembly. To investigate the multimeric assembly of MU1140-lipid II and the behavior of water permeation, I set up additional systems with two and four MU1140-lipid II complexes in the bacterial membrane. Both systems were run for $500 \mathrm{~ns}$ each. For each set up, MU1140-lipid II complexes were placed in the membrane with lipid II chains facing away from each other and MU1140 facing towards each other (Figure 4.7). I observed that the MU1140 chains drifted towards each other and water molecules permeated across the lipid bilayer. Movie $\mathrm{S} 2^{72}$ shows this process for the four-chain system. In Figure 4.7, I highlighted (with red spheres) the water molecules within $3.5 \AA$ of MU1140 residues with red spheres. Before the simulation, only a few water molecules above the upper lipid layer are in the vicinity of MU1140. At the 
end of the $500 \mathrm{~ns}$ simulations, both the two-chain system and the four chains systems show significant water in the transmembrane region. Interestingly, the lipid molecules near the MU1140-lipid II complexes were pulled inward. The four MU1140-lipid II complexes show a significant shrinkage or distortion in the membrane thickness (Figure 4.7b, c). Due to the short length of MU1140, the membrane is noticeably tapered near the MU1140-lipid II assembly. This is also displayed by the number density of water molecules which shows a narrowing of the density profile with the increase in the number of MU1140 chains (Figure $4.7 \mathrm{~d}$ ). Figure $4.7 \mathrm{~d}$ also shows a significantly higher population of water molecules in the transmembrane region for the two and four complexes. An independent, additional set of simulations for one, two, and four complexes yielded quite similar results of water density profile as well hydrogen bonding pattern (Fig. S3) ${ }^{72}$. Here, the number density of water molecules is calculated using the density profile plugin in $\mathrm{VMD}^{99}$ and represents the number of atoms of water divided by the total number of atoms in the system projected along the z-axis. The displayed number densities are obtained by averaging over the last $300 \mathrm{~ns}$ of the MD trajectories. The membrane distortion/tapering and lipid relocation towards the central region of the lipid bilayer due to the MU1140 assembly in the membrane suggests that a higher concentration of the peptide will cause significant disruption of the bacterial membrane, and provides additional support to the model of Matsusaki-Huang which previously proposed a membrane-thinning effect before the actual poration process takes place for amphiphilic peptides. ${ }^{63}$ 
a)
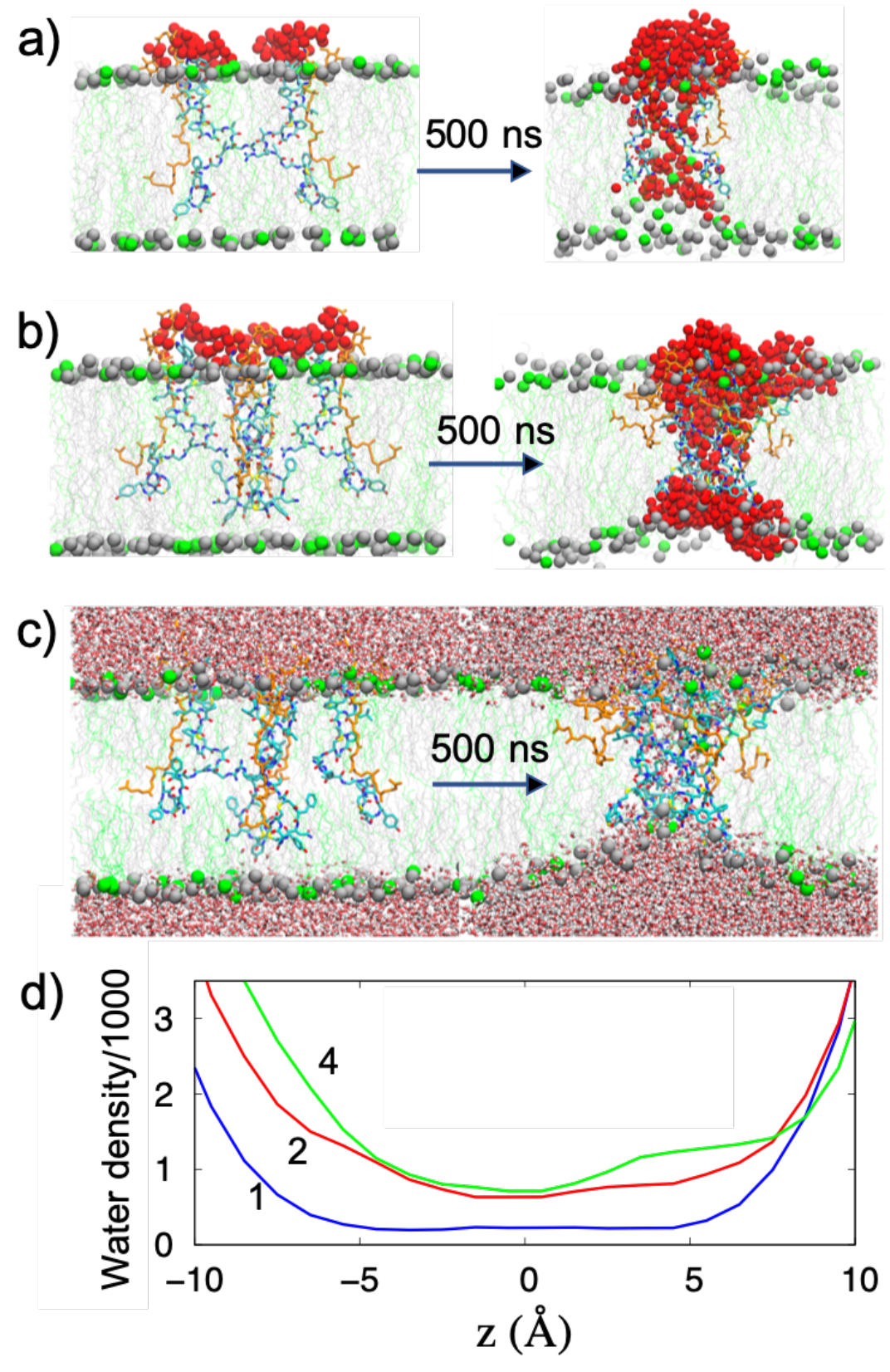

Figure 4.7 Assemblies of a) two and b) four complexes of MU1140-lipid II in the bacterial membrane at the beginning and end of the $500 \mathrm{~ns}$ simulations. MU1140 chains are colored by residue types, lipid II chains are highlighted in yellow, and the water molecules within $3.5 \AA$ of MU1140 are highlighted as red spheres. As in Figure 4.4, the phosphorus atoms of POPE are shown as green spheres and POPG phosphorous atoms as gray spheres. c) Same as b) but showing the water molecules above and below the lipid layers. The membrane is tapered near the MU1140-lipid II assembly due to the short length of MU1140. d) Density profile of the water molecules in the transmembrane region of the lipid bilayer for one, two, and four complexes of MU1140-lipid II. 
For both the two and four complexes, significant hydrogen bonding between the water molecules and MU1140 is observed as shown in Figure 4.8. For these complexes, I calculated the percentage hydrogen bonding with water per chain and plotted the result as a bar graph in Figure 4.8c. Compared to almost non-existent hydrogen bonding with Ala11 and Ala16 with in a single chain of MU1140 in the membrane, both the two and four chain complexes showed markedly higher hydrogen bonding with these residues. Similarly, Arg13 also showed an increased hydrogen bonding in the two and four MU1140-lipid II complexes. Hydrogen bonding with other residues are found to be similar among systems with one, two, and four complexes. Interestingly, the space between the two and four MU1140 chains allows multiple water molecules to traverse the transmembrane region at the same time, and thus breaks the single-file nature of the water permeation that occurs in only chain (Figure 4.5). I calculated the first passage times for successfully traversing the transmembrane region and plotted their distributions. It is interesting to note that the first passage time distribution for one MU1140-lipid II complex has only one well-defined peak with $\langle t\rangle=23 \mathrm{~ns}$ but the distribution for two and four complexes seem to show two different timescales for the water traversing the lipid bilayer: 1) one peak around $22 \mathrm{~ns}$, which is similar to the one chain MU1140 system and likely represents the single-file transportation and 2) the other peak at a much faster timescale $\sim 10 \mathrm{~ns}$, likely from the diffusion in the mid-region. 


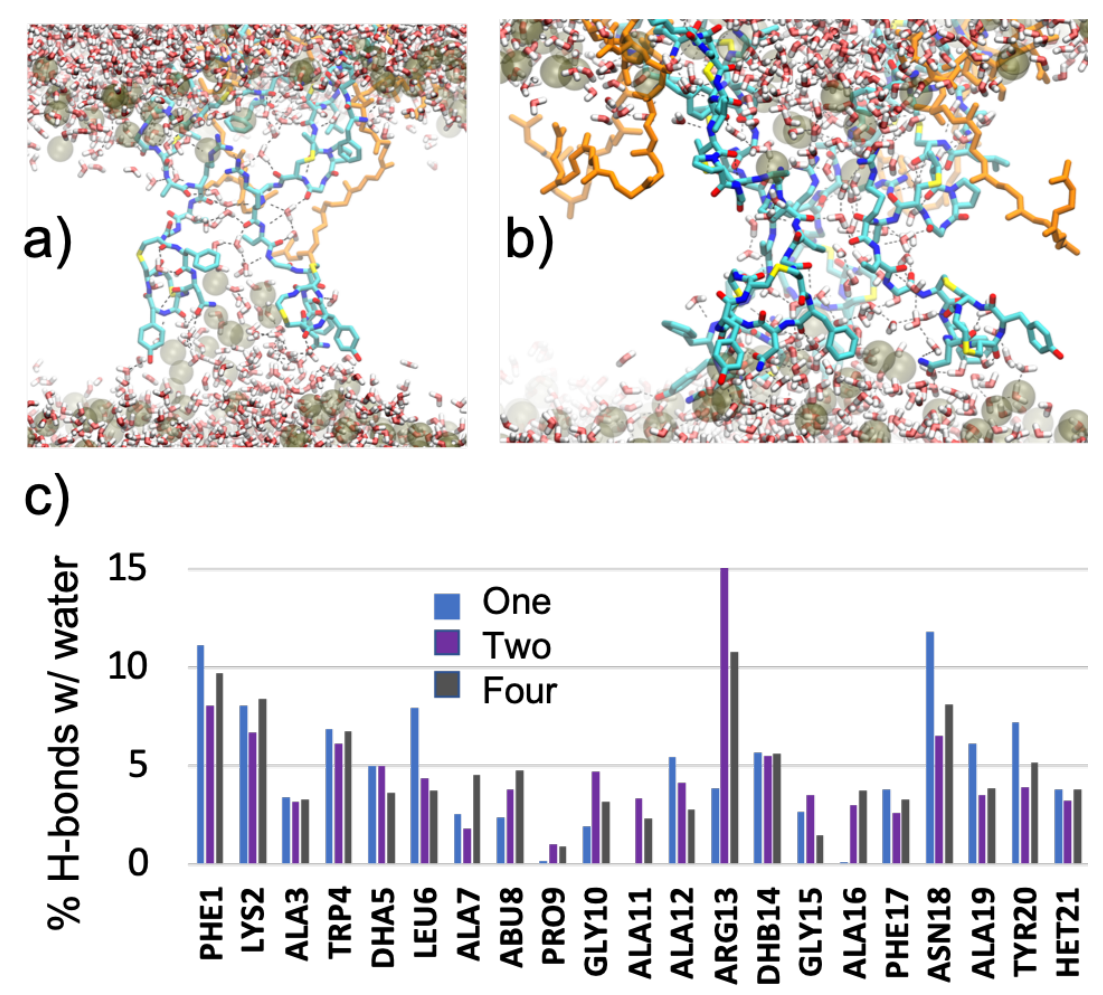

Figure 4.8 Representative snapshots of a) two and b) four MU1140-lipid II complexes showing water permeation. MU1140 chains are shown in cyan color and lipid II chains are shown in orange. For clarity, only the phosphate atoms of the lipids are displayed. C) Percentage of hydrogen-bonding per chain for MU1140 and water in the transmembrane region for one, two, and four complexes of MU1140-lipid II.

4.2 A Comparative Study of The Lipid II Binding and Transmembrane Properties of Antimicrobial Lanthipeptides.

In this study, I use atomic-scale molecular dynamics (MD) computational studies to compare both the lipid II binding ability and the membrane water channel formation mechanisms and efficacy of five different lantibiotics that are part of the focus of current antimicrobial research. They are nisin produced by Gram-positive bacteria Lactococcus lactis $^{122}$, Mutacin 1140 (MU1140) produced by Streptococcus mutans ${ }^{54}$, gallidermin produced by Staphylococcus gallinarum $^{123}$, NVB302 isolated from Actinoplanes 
liguriae $^{I 24}$, and NAI107, also known as microbisporicin or 107891, produced by the actinomycete Microbispora $^{125}$. Where, NVB302 and NAI107 are chemically modified semi-synthetic lantibiotics ${ }^{125-127}$ (REF). Our results indicate that all five of these peptides bind with the lipid II molecule, and also have the potential to form membrane water channels however, the relative abilities vary dramatically. Among the five, nisin is found to be the most effective water channel former. Gallidermin and MU1140 are found to bind more strongly to lipid II molecules than the others. The shorter peptide NVB302 has the interesting ability to taper the membrane bilayer which can lead to membrane disruption. These detailed observations provide insight into the dynamics of membrane pore-forming peptides $^{6,29}$ and can facilitate the design and development of especially effective antimicrobial agents.

\subsubsection{Molecular Models of Peptide-Lipid II Complexes}

Nisin is composed of 34 amino acids, NAI107 is composed of 23 amino acids, MU1140 and gallidermin are each composed of 21 amino acids, and NVB302 is composed of 19 amino acids (Figure 4.9). The crystallographic structure of the nisin-lipid II complex was taken from the Protein Data Bank (PDB ID 1wco), and that of NAI107 was from PDB ID 2mh5. The structure of the MU1140-lipid II complex was taken from our earlier publication ${ }^{72}$. This structure was used as a template to construct the 3D structure of NVB302-lipid II and gallidermin-lipid II. Since the interaction region is the pyrophosphate moiety of lipid II, I used a dipeptide (Ala, Glu) as a peptide moiety and a longer bactoprenol tail was used to provide a stable anchorage during membrane-lipid II simulation. Figure 4.9 displays a schematic (left) and 3D structure (right) of the five different peptide-lipid II complexes taken after $10 \mathrm{~ns}$ of MD simulation in solution with no membrane. 


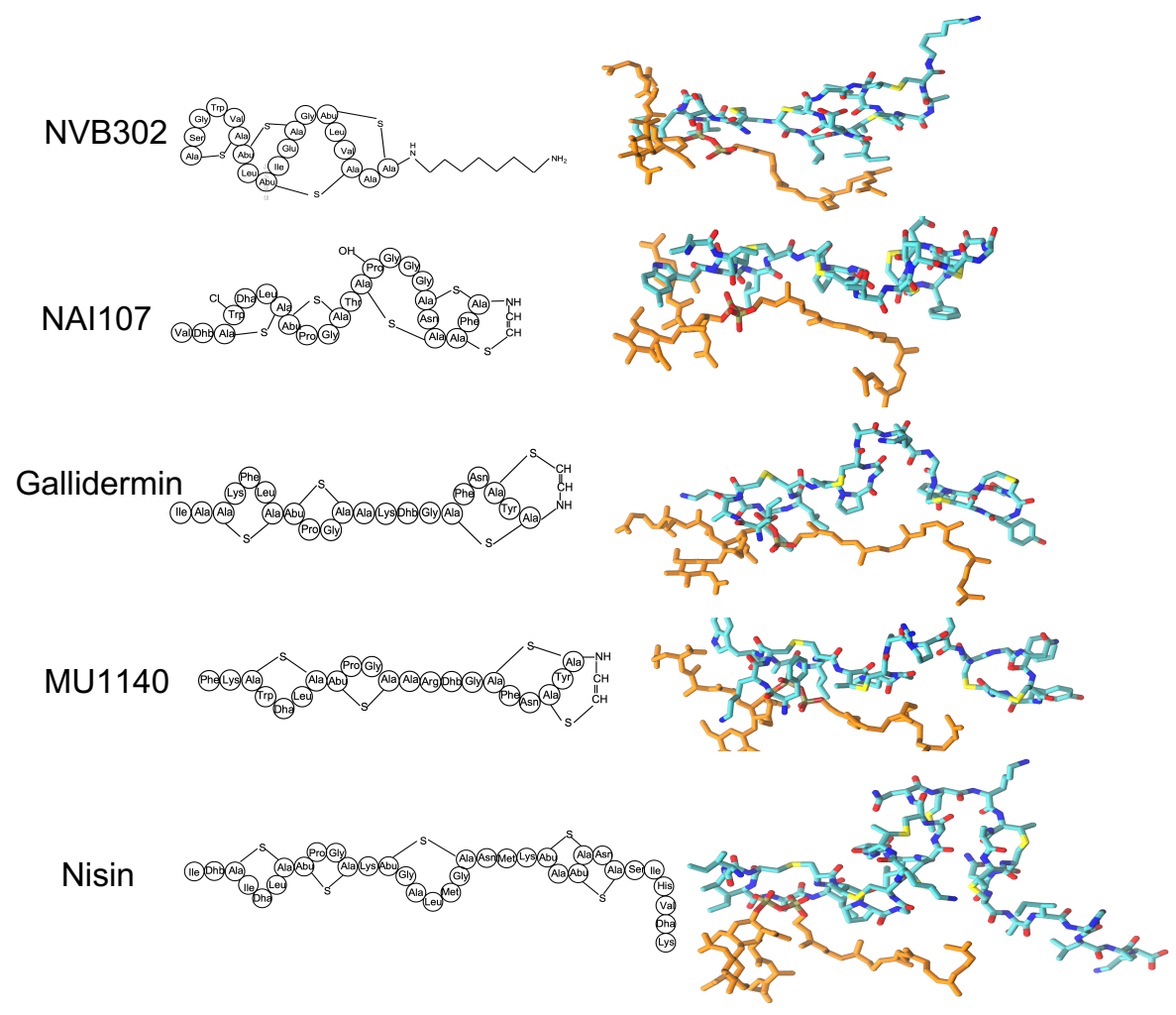

Figure 4.9 (Left) Schematic representation of the amino acid sequence for the lantibiotic variants. (Right) Their modeled structure after a $10 \mathrm{~ns}$ MD simulation with lipid II (orange) in solution, with no membrane.

The structures displayed in Figure 4.9 show that the number of saturated thioether rings is three for gallidermin, three for MU1140, four for NVB302, four for NAI107, and five for nisin. In common, MU1140, gallidermin, and NAI107 each have unsaturated AviCys ring at the $C$-terminus. The net charge of these peptides, excluding $N$-terminus, is: nisin (+3), MU1140 (+2), gallidermin (+2), NAI107 (0) and NVB302 (0). A net positive charge helps a peptide to engage with the negatively charged bacterial membrane surface ${ }^{6-}$ 7,104. The positively charged nisin, MU1140, and gallidermin have a flexible hinge region before the last two rings comprised of three, four, and four amino acids, respectively. That flexibility helps them to insert into the bacterial membrane to form membrane pores. The 
neutral NAI107 is unique with the presence of a chlorinated TRP at residue 4 and a hydroxyproline (HYP) at residue 14. The two neutral peptides NVB302 and NAI107 have a more globular structure and hence it is of interest to investigate their behavior inside the bacterial membrane.

\subsubsection{Membrane Tapering}

Previous studies ${ }^{63-64,72,128}$ demonstrated the pore forming ability of nisin, gallidermin, and MU1140. For shorter lantibiotics like gallidermin, the pore formation depends upon membrane thickness. However, there are no studies reporting on the pore formation activities of Type B lantibiotics like NAI107 and NVB302. To investigate and compare the mechanism of channel formation, I placed all five protein-lipid II complexes vertically inside the membrane. Figure 4.10 shows snapshots of the initial and final structures from the $500 \mathrm{~ns}$ simulation for each of the peptide-membrane system. For the initial set-up, I exposed only the $N$-terminal to water, and I placed the $C$-terminal inside the membrane. As the MD computations evolved, within the first $100 \mathrm{~ns}$ the $C$-terminal of all peptides reached the other surface of the membrane and became exposed to water. This spanning of the membrane allowed all five peptides to create a water channel (red spheres in Figure 4.10). The shortest peptide NVB302, has an interesting mechanism for spanning the membrane; it pulls the membrane surfaces inward towards each other so that less distance is required of the peptide to span the membrane to expose both ends of the peptide to water. 


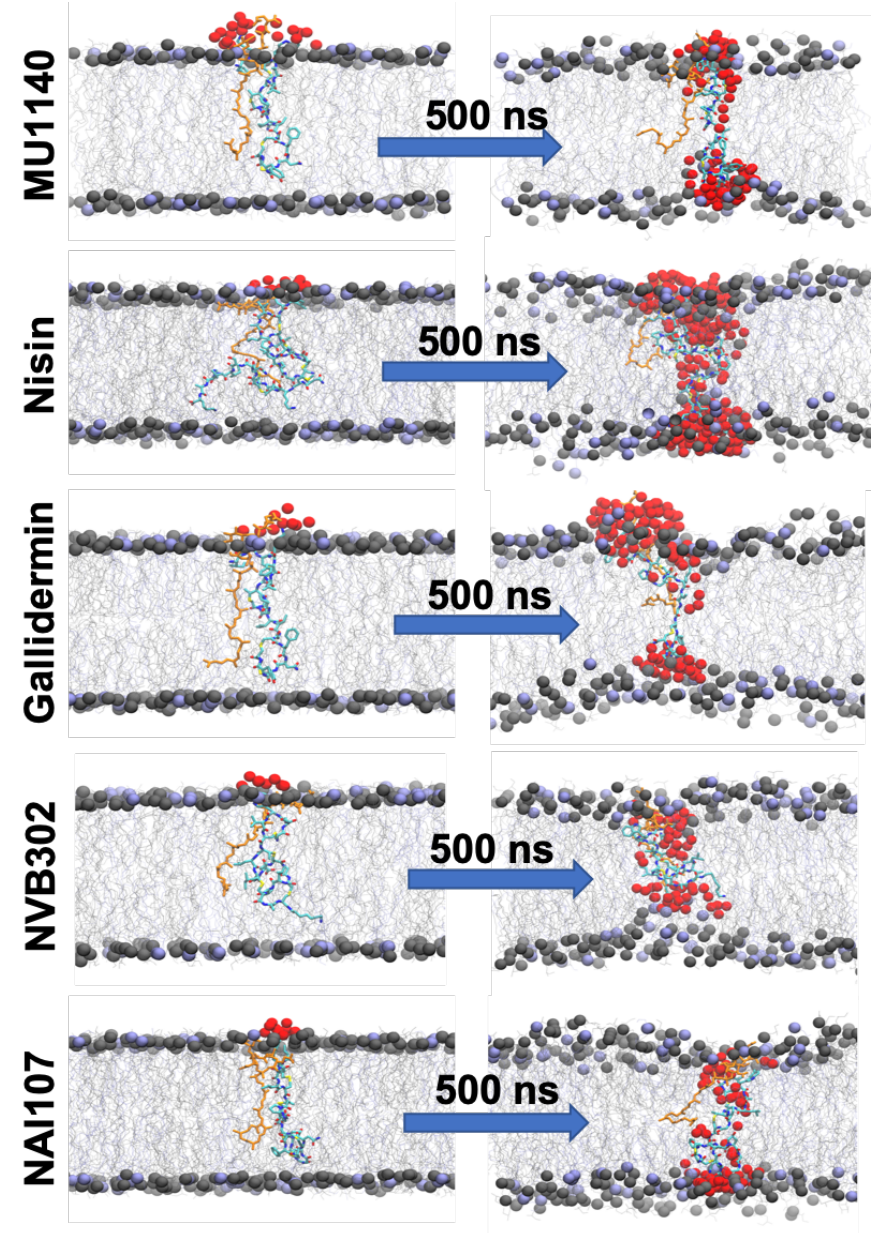

Figure 4.10 Snapshot of the protein-lipid II membrane complexes: (left) initial set-up, and (right) after $500 \mathrm{~ns}$ of MD computational simulation. The bacterial membrane is displayed in gray and the gray spheres are the phosphate atoms on the two surfaces of the lipid bilayers. Red spheres are water molecules within $5 \AA$ of the peptides.

To quantify the membrane thinning caused by each peptide, we calculated the average position of the phosphate atoms within $12 \AA$ of the peptide on the upper membrane surface, and the same for the phosphate atoms within $12 \AA$ of the peptide on the lower membrane surface. Figure 4.11 displays the separation of the membrane surfaces averaged over the last $300 \mathrm{~ns}$ of the MD computational simulation. For reference, the control thickness for the lipid bilayer without containing a peptide-lipid II complex is 
approximately $40 \AA$. It is observed that all the peptide complexes pull the membrane surfaces inward. The thinning effect is least for MU1140 and gallidermin, moderate for nisin and NAI107, and most pronounced for NVB302. Though nisin is the longest peptide, it has quite a high membrane thinning effect. The presence of more hydrophilic residues at the $C$-terminal region, especially the Lys residues, and absence of AviCys ring may contribute to the pulling of the phosphate atoms. The pronounced squeezing of the membrane by the shorter and globular lantibiotics NVB302, NAI107 assists their ability to form membrane water channels once they insert into the membrane.

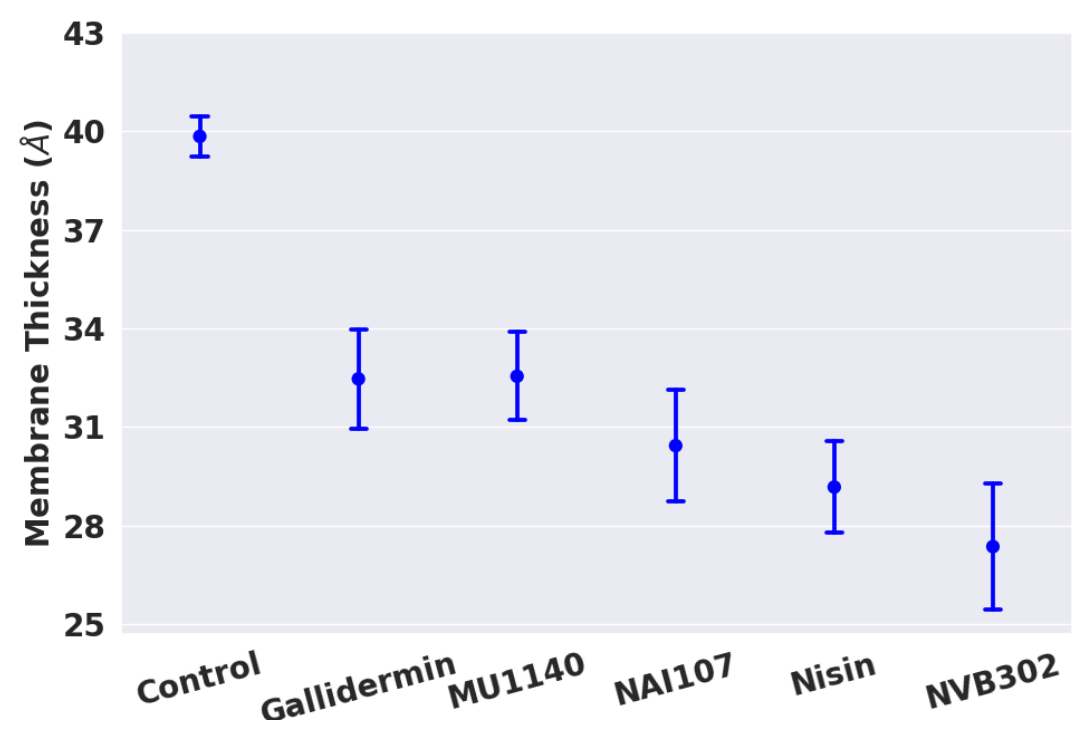

Figure 4.11 Membrane thickness Average distance between phosphate atoms of the top and bottom of the membrane within $12 \AA$ of peptides.

\subsubsection{Channel Formation: Insertion and Transport of Water Across the Membrane}

\section{A. Water Insertion}

Figure 4.10 shows that all the peptide-lipid II complexes form a water channel (red spheres) through the membrane. To investigate the effectiveness of the membrane channels, I calculated the number density of water molecules across the membrane along 
the lantibiotic peptide. The number density of water molecules was calculated using the density profile plugin in $\mathrm{VMD}^{99}$, which calculates the concentration of water molecules per length $(\AA)$ along a z-axis that is aligned along the peptide. Figure $4.4 \mathrm{a}$ is a density profile of water molecules within the membrane region from $z=-10$ to $+10 \AA$, averaged over the last $300 \mathrm{~ns}$ of the MD simulation. This $20 \AA$ segment of the channel spans the membrane center that is at $\mathrm{z}=0$. I chose to examine the water molecules along this inner region of the channel's z-axis because it avoids the piling-up of bulk water molecules at the membrane surface. Figure 4.12 shows that the water number density at the center of the channel is highest for nisin and lowest for gallidermin. It is interesting to see a relatively high density of water for NVB302, even though it has the least number of residues. This effectiveness in creating a channel may be related to the ability of NVB302 to significantly taper the bacterial membrane, as seen in Figure 4.11. I also plotted the time evolution of the number of hydrogen bonds formed by these lantibiotics with water molecules (Figure 4.12b). Consistent with its large water density, nisin has the highest number of hydrogen bonds. Nisin is the longest of the peptides and has a more positive charge than the other peptides. MU1140, NVB302, and gallidermin have approximately the same number of hydrogen bonds. NAI107 has the least number of hydrogen bonds with water, possibly related to its long, hydrophobic $C$-terminal tail. 

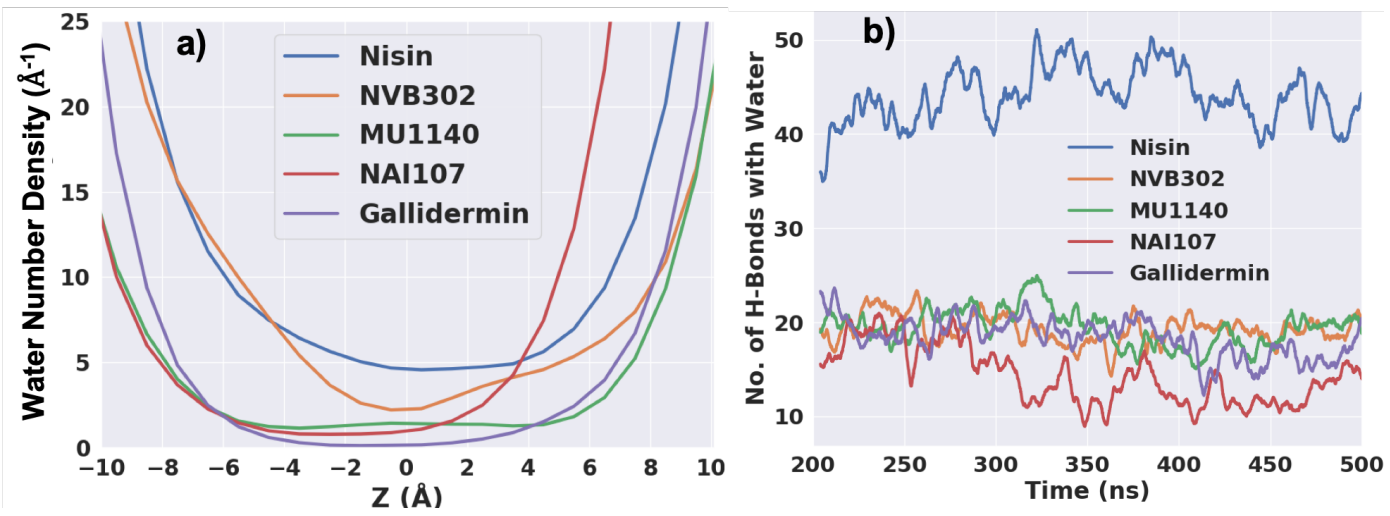

Figure 4.12 For the protein-lipid II complexes: a) Density profile of the water molecules along the z-axis of the transmembrane region of the lipid bilayer, b) During the last 300 ns of the MD simulation, the time evolution of the number of $\mathrm{H}$-bonds made by each peptide with water molecules.

\section{B. Water Transport}

Though the number density of water molecules is a good measurement for the degree of water insertion inside the membrane, it does not directly measure the actual flux of water molecules across the membrane. Hence, I also calculated the average number of water molecules passing across the membrane from either side of the membrane during the last $300 \mathrm{~ns}$ of the MD simulation. The results are plotted in Figure 4.13a. The relative effectiveness of a peptide to enable water passage displayed in Figure $4.13 \mathrm{a}$ is approximately the same as the relative effectiveness of allowing water insertion into the membrane displayed in Figure 4.12a. Gallidermin has the least number of water molecules (approximately 10) passing across the membrane. Figure $4.13 \mathrm{a}$ does not display any notable directional preferences of water molecules passage through the membrane. I also plotted the average passage time of water molecules across the membrane (Figure 4.13b). For this calculation, I set the two ends of the membrane region to be at the $\mathrm{z}$ position of the average position of the phosphate atoms of lipids in the lower and upper surfaces of the 
untapered membrane, which are $z=-15 \AA$ and $z=+15 \AA$, respectively. Consistent with nisin's ability to allow water insertion into, and passage across the membrane, nisin also displays the shortest time for water traversal across the membrane, which is further evidence of the effectiveness of nisin in membrane channel formation.
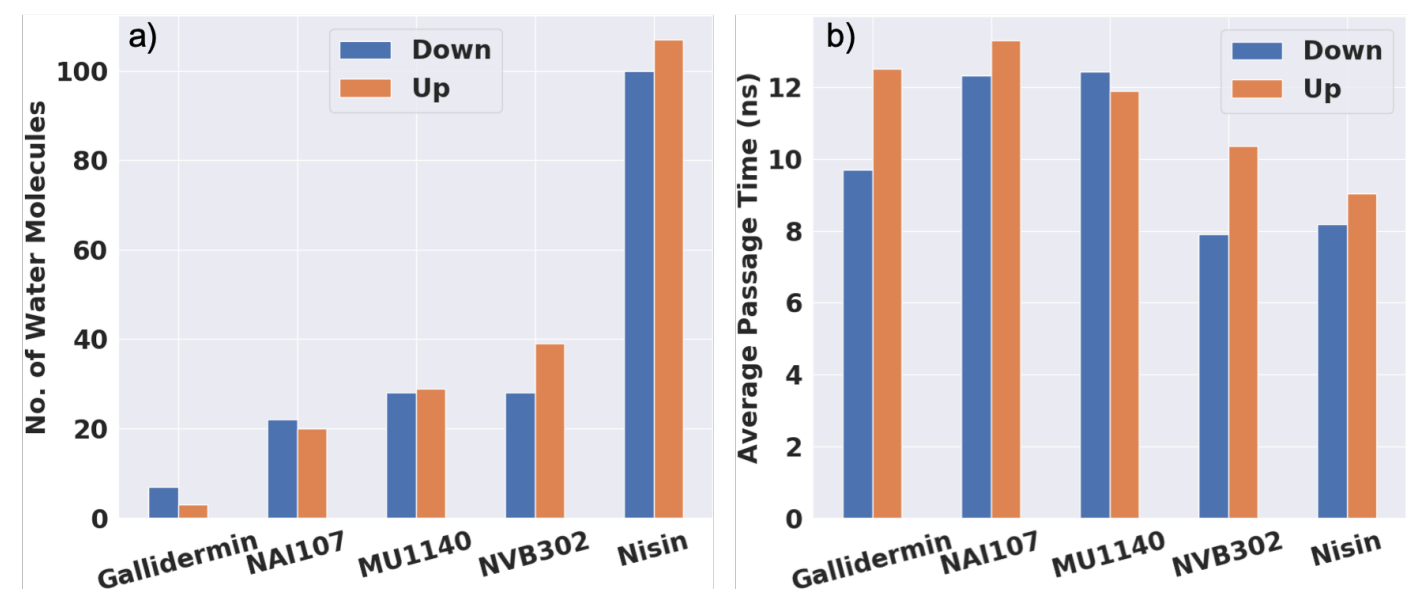

Figure 4.13 For each of the peptide-lipid II complexes: a) Number of water molecules passing across the membrane from either direction summed over the last $300 \mathrm{~ns}$ of the MD simulation, b) Average passage time of a water molecule to cross the membrane region. "Up" and "Down" refers to the average position of the phosphate atoms of lipids in the lower and upper surfaces of the untapered membrane.

\section{Peptide Hydrogen Bonding with Water}

Especially important for single-file water transport across a membrane that is facilitated by a single peptide is hydrogen bonding ${ }^{117,129}$ between water molecules and peptide amino acid residues. For each peptide, I determined the percentage of time during the MD simulation that each residue makes a hydrogen bond with a water molecule. The results are plotted in Figure 4.14. Since an amino acid can simultaneously make multiple hydrogen bonds to multiple water molecules, the percentages can be greater than $100 \%$. A major contribution to hydrogen bonding for most residues in all the lantibiotics is from the $\mathrm{CO}$ (red bars) and NH (blue bars) groups of the backbone. In addition, hydrophilic residues 
also display significant hydrogen bonding of water molecules with their side chain (SCgreen bars). The side chain hydrogen bonding is especially noticeable for nisin, which has the largest number of hydrophilic residues in the interior of the membrane. It is also seen that a peptide's ability to draw water into the membrane (Figure 4.12a) and facilitate its passage across the membrane (Figure 4.13a), is correlated with the hydrogen bonding ability of the peptide (Figure 4.12b and Figure 4.14). Figure 4.12b and Figure 4.14 show that nisin makes the most hydrogen bonds with water, and especially with hydrophilic amino acids in the interior of the membrane. Figure $4.12 \mathrm{a}$ shows that this results in the largest number of water molecules in the interior of the membrane, and Figure 4.13a shows that nisin has the largest number of water molecules traversing the membrane. Figure $4.13 \mathrm{~b}$ also shows that nisin has the fastest passage time for water molecules across the membrane. Thus, a strategic placement of hydrophilic residues along the peptide sequence can facilitate water passage through the membrane. 

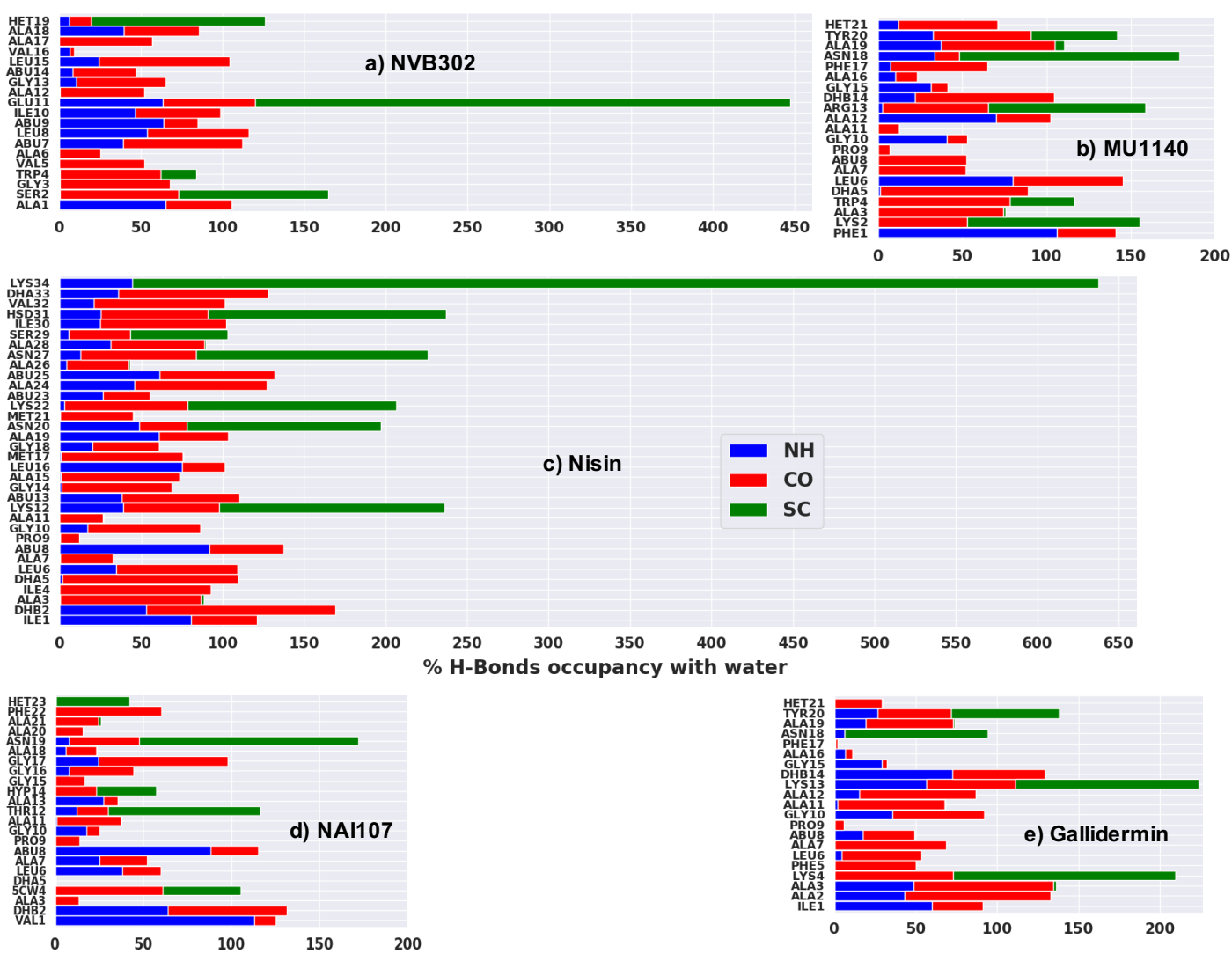

Figure 4.14 Percentage of hydrogen-bonding between peptide residues and water molecules, showing residual hydrogen bonds with backbone $\mathrm{CO}$ (red), backbone NH (blue), and sidechain (SC) (green).

More details are given in Table 4.1, which provides a quantitative comparison of the percentage contribution of the three groups $(\mathrm{CO}, \mathrm{NH}$, side chain) for all five lantibiotics. Averaged over all peptides, the percentage contribution to hydrogen bonding with water molecules from the backbone CO group is approximately 50\%, with NAI107 having the lowest at $45 \%$. The $\mathrm{NH}$ contribution averages $25 \%$ and is a maximum for NAI107 at 31\%. The side chain (SC) contribution to hydrogen bonding with water molecules averages $25 \%$, and is largest for nisin at $31 \%$, which is expected because nisin contains more hydrophilic residues than the other peptides. 


\begin{tabular}{|l|c|c|c|}
\hline \multirow{2}{*}{ Lantibiotics } & \multicolumn{3}{|c|}{ \% contribution to H-bonds with water by } \\
\cline { 2 - 4 } & $\mathrm{NH}$ & $\mathrm{CO}$ & $\mathrm{SC}$ \\
\hline Gallidermin & 25 & 52 & 23 \\
\hline NAI107 & 31 & 45 & 24 \\
\hline MU1140 & 26 & 52 & 22 \\
\hline NVB302 & 22 & 49 & 29 \\
\hline Nisin & 21 & 47 & 32 \\
\hline
\end{tabular}

Table 4.1 Percentage contribution of peptide residues' NH, CO, and SC hydrogen bonding with water.

The correlation between hydrogen bonding and water passage is further displayed by additional information contained in Figure 4.14 and Table 4.1. Figure 4.12a and Figure 4.13a show that gallidermin is least successful in drawing water into the membrane and facilitating its passage. Figure 4.14 and Table 4.1 show that gallidermin is the only peptide that has two different amino acids with less than 10\% occupancy of hydrogen bonds, PHE17(2\%) and PRO9(6\%). In contrast, nisin, which is most successful in drawing water into the membrane and facilitating its passage does not have any amino acids with less than $10 \%$ hydrogen bond occupancy. The other three peptides have only one amino acid with less than $10 \%$ hydrogen bond occupancy and their water passage facilitation falls between gallidermin and nisin. Thus, just as large hydrogen bonding facilitates water passage through the membrane, especially low hydrogen bonding by amino acid residues acts as a roadblock to water passage. This is also seen in Figure 4.15, which displays for water molecules that cross the membrane the average time that they spend near each residue ( 2.5 $\AA$ ). The residues in Figure 4.14 that have especially small hydrogen bonding with water lead to long delay times (bottlenecks) nearby, as seen in Figure 4.15. 


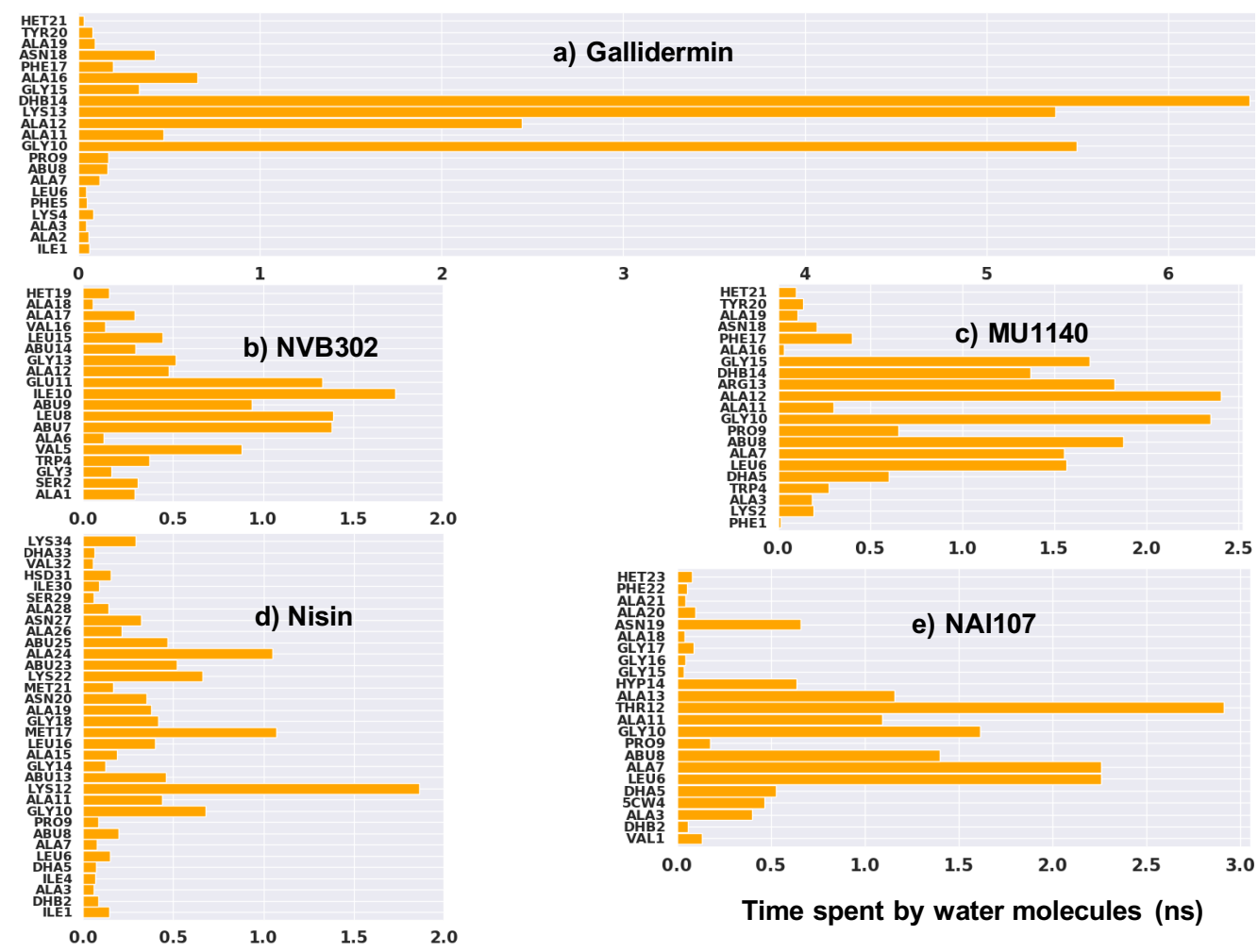

Figure 4.15 Average time spent by water molecules near each residue (within $2.5 \AA$ ).

\section{Water Passage Trajectories}

Earlier studies suggested that other lantibiotics do not form pores as effective as nisin because they are shorter and could not span the membrane ${ }^{63}$. Moreover, these studies emphasize that the rigid, globular structure of some lantibiotics could be an especially big hindrance to spanning the membrane and forming membrane pores ${ }^{59,130}$. To study the membrane-spanning ability of our five lantibiotics, I calculated the average structure of the protein-membrane system from the last $300 \mathrm{~ns}$ of the MD simulation. The results are displayed in the left column (I) in Figure 4.16. The upper and lower orange lines are drawn at $\mathrm{z}=10 \AA$ and $\mathrm{z}=-10 \AA$, respectively. Outside of this region, I observed bulk water molecules. The gray circles represent the phosphate atoms at the surface of the lipid 
bilayers. In the middle column (II), I plotted the trajectories of all water molecules that successfully traverse the membrane during the last $300 \mathrm{~ns}$ of the MD simulation. Column III (right panels) of Figure 4.16 display the density of successful water trajectories projected onto the $(x-y)$ plane.

Figure 4.16 shows that though the gallidermin and MU1140 both have 21 residues and similar rings, MU1140's amino acid sequence allows it to span the membrane while gallidermin does not, which hinders water transport. There are relatively few successful water trajectories for gallidermin and a relatively small region in the $x-y$ plane that successful water trajectories can navigate. A bend in the gallidermin structure at the hydrophilic Lys13 residue prevents the peptide from elongating enough to reach the lower leaflet and hence it functions poorly as a channel-forming peptide. In contrast, MU1140 contains the hydrophobic residue Trp4 in place of Lys4. The hydrophobic Trp4 is able to easily assume different conformations in the hydrophobic environment inside the membrane and therefore allows the $C$-terminal of MU140 to stretch to the lower surface of the membrane. Among the two globular peptides, NVB302 forms more efficient channels than NAI107, though both are capable of forming membrane channels via tapering the membrane. Though NVB302 is the shortest of the peptides, it is the most effective at tapering the membrane (Figure 4.11). Due to the wider cross-section of NVB302 around the second and third thioether rings, it can provide multiple pathways for water molecules to pass across the membrane (Column III). Nisin, the longest of the peptides, easily spans across the membrane. Flexible kinks in the structure provide multiple paths for water (Column II). Interestingly, Column III shows that nisin provides a reasonably wide number of trajectories, but not the widest range of trajectories of the peptides. However, as seen in 
Figure 4.15, nisin has the fewest number of roadblock delay points along its structure. This combination of a mid-level number of different trajectories along with no significant delay points results in nisin being the most effective of the peptides in facilitating water transport across the membrane.

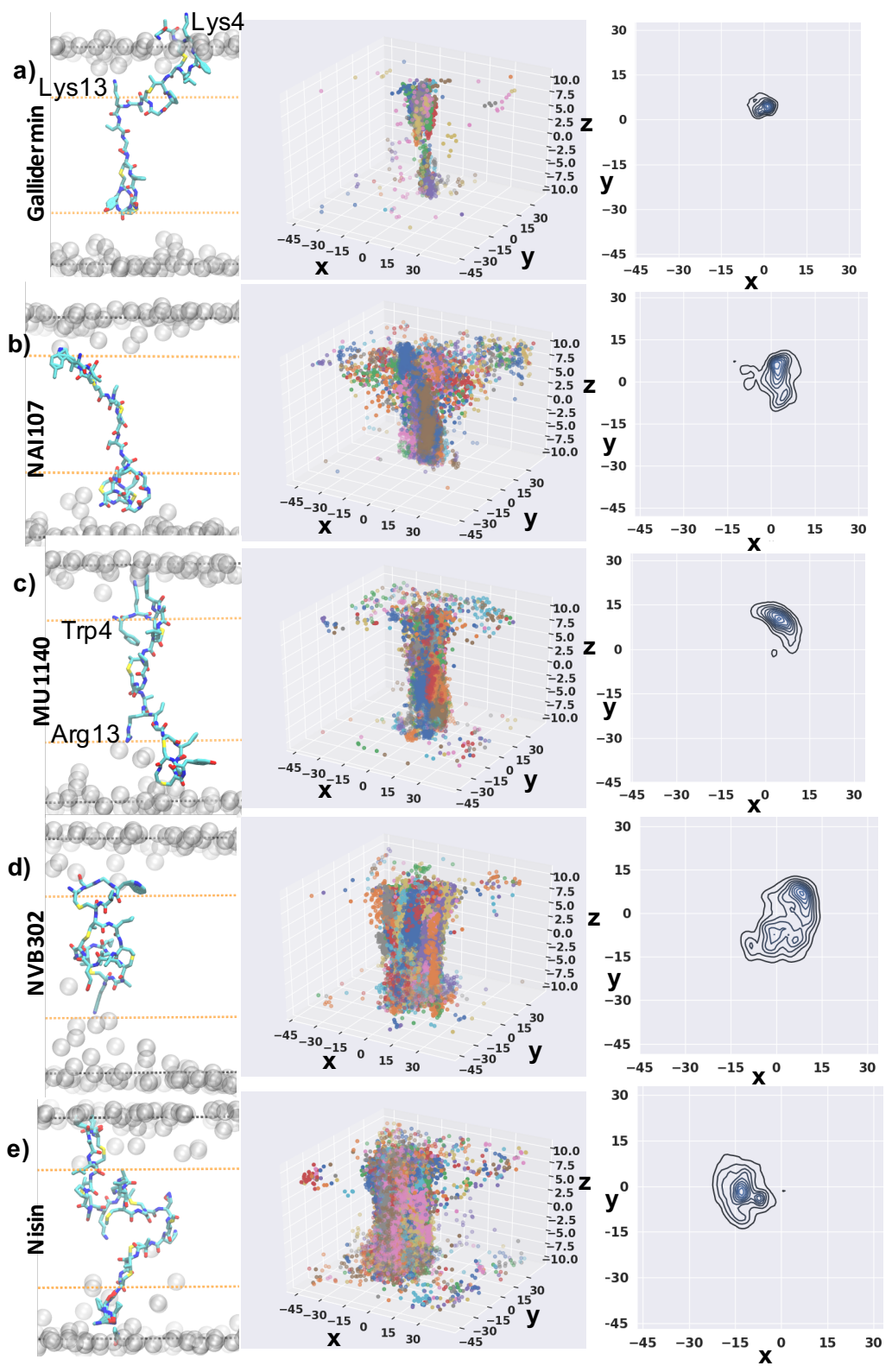

Figure 4.16 For the last $300 \mathrm{~ns}$ of the MD simulation: Column I) Average position of peptide (Cyan) and the phosphate atoms (gray) of the membrane surfaces. (II) Trajectories of all water molecules that successfully cross the membrane (colors represent distinct water 
molecules). (III) Two-dimensional density plot along the x-y plane of the projection of the trajectories in Column II.

\subsubsection{Interaction with Lipid II}

The water channel formation ability of lantibiotics discussed above may be a novel mechanism involved with membrane disruption that can contribute to their bacteriostatic or bacteriocidal properties. Another disruptive mechanism involves the interaction of a peptide with lipid II molecules in the membrane. This mode of action requires the peptide to first engage and bind with the pyrophosphate moiety of lipid II molecules before forming

membrane pores ${ }^{59,65}$. To investigate this mechanism, I calculated the total energy of interaction between peptides and lipid II molecules. The total energy is the sum of the electrostatic interaction and Van der Waals interaction. Figure 4.17 shows that gallidermin and MU1140 have the strongest total energy of interaction with lipid II molecules. NAI107 has the weakest interaction energy. Though nisin is the most efficient for transmembrane water transport, its interaction with lipid II is notably weaker than that of gallidermin and MU1140. I also calculated the number of hydrogen bonds made by these five peptides with the pyrophosphate moiety of lipid II molecules during the last 300 ns of the MD simulation. The results are plotted in Figure 4.17b and the ordering of the peptides is consistent with the interaction energies of Figure 4.17a. Figure 4.17c depicts the important residues in each peptide that make hydrogen bonds with the lipid II molecule. 

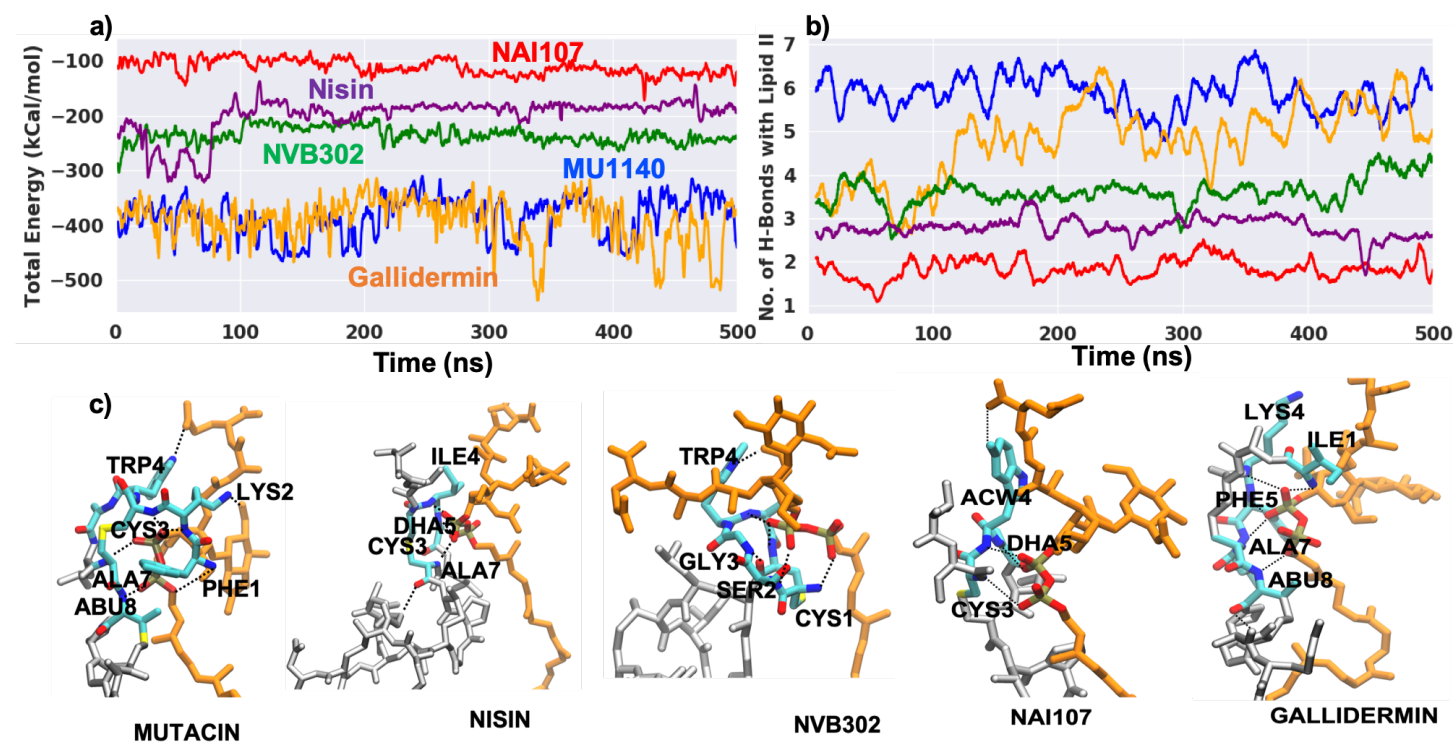

Figure 4.17 a) Total energy (Elec+VDW) of interaction between peptide and lipid II molecule. b) The number of H-bonds made by each peptide with a lipid II molecule. c) Important residues (blue) in each peptide that make hydrogen bonds with the lipid II molecule (orange).

The graphs plotted in Figure 4.18 quantify the hydrogen bonding occupancy of the residues highlighted in Figure 4.17 during the last 300 ns of the MD simulation. MU1140 has the highest occupancy with more than $60 \%$ for five residues (Lys2, Trp4, Abu8, Ala7, and Phe1). The next is gallidermin with four residues (Ile1, Ala7, Lys4, Phe5) having more the $60 \%$ bonding occupancy. NVB302 has two residues (Ser2, Trp4), nisin has three residues (Ala7, Ile4, Ala3), and NAI107 has just one residue (5cw4) having more than $60 \%$ hydrogen bonding occupancy. Figure 4.18 also shows that the $\mathrm{NH}$ group from the backbone of these residues has the chief role in forming H-bonds with lipid II molecules. These residues come from the first and second thioether ring of all the peptides that form the binding cage for the pyrophosphate moiety. 

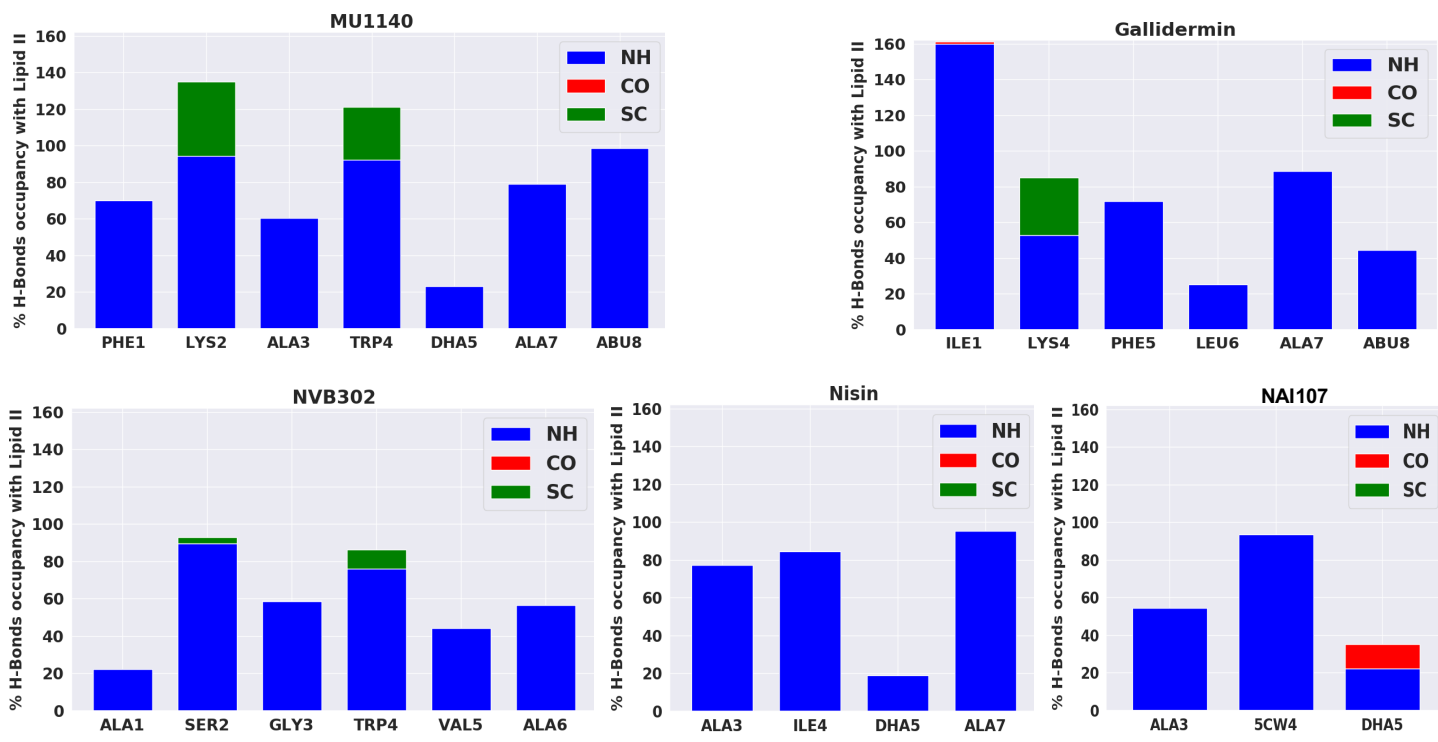

Figure 4.18 Hydrogen bond occupancy percentage between lipid II molecules and the peptide residues (for the last $300 \mathrm{~ns}$ of the MD simulation) highlighted in Fig. 4.17. Hydrogen bonds with peptide backbone CO (red), peptide backbone NH (blue), and side chain (green).

The results displayed in Figure 4.17 and Figure 4.18 show that even though gallidermin is ineffective in facilitating water transport through the membrane, gallidermin may be especially effective in disrupting cellular structure by interacting strongly with lipid II molecules. This is also true for MU1140. In contrast, nisin is especially effective in facilitating water transport, but interacts relatively weakly with lipid II. 


\section{DESIGNING HYBRID LANTHIPEPTIDES FROM MUTACIN 1140 AND EBOLA}

\section{VIRUS DELTA PEPTIDE FOR IMPROVED DRUG PROFILE}

Though there are many ways of managing the antibiotics resistance crisis, there is an urgent and significant need for a new class of antibiotics to mitigate the threat of antibiotic-resistance ${ }^{131}$. An important strategy to tackle resistant strains is to develop therapeutic agents having multiple modes of inhibitory mechanisms ${ }^{132-134}$. Such multiaction or hybrid antibacterial agents provide advantages, including a broad range of activity, difficulty for bacterial spontaneous mutation and resistance development, reduced toxicity, and improved efficacy than $1+1$ combinations of the individual constituent agents $^{135}$. Current hybrid antibiotics in research studies or clinical evaluations include the super molecules formed by merging the features of two or more antibiotics, and hybridization of antibiotics with non-antibacterial molecules ${ }^{134-138}$.

As described earlier, I explored the interactions of MU1140 with lipid-II and its mechanism of membrane pore formation in the Gram-positive bacterial membrane using molecular dynamics (MD) computational simulations. The MU1140-lipid II complexes can form functional, water permeating membrane pores, and the anionic pyrophosphate moiety of lipid-II provides the binding target for the first two thioether rings in MU1140. The novel mode of action for MU1140 and other lantipeptides can potentially be exploited to develop optimized peptide variants with improved antimicrobial properties and therapeutic profile. However, the ability for membrane insertion of a peptide and oligomeric pore assembly and stability in the hydrophobic transmembrane region comes with a reduction in its solubility, thereby limiting the drug delivery and efficacy. In addition to low solubility, also low production yield, proteolytic degradation, and susceptibility to 
oxidation are major limitations that decrease the druggability of lantibiotic peptides ${ }^{135,139-}$ 141. The low production yield is mainly due to the elusive and cumbersome process of synthesizing the AviCys moiety ${ }^{142-143}$, and it is a major hindrance for widespread usage ${ }^{139}$, 144. Escano et al. in 2017, reported that the AviCys ring of MU1140 is necessary for the lateral assembly mechanism of activity that traps lipid II into a large complex, and getting rid of the ring reduces the bioactivity of the peptides. Their study suggested that the loss of activity of the AviCys devoid variant MU1140-COOH can be restored by simple amine capping ${ }^{143}$. Another excellent example of modifying the C-terminal for improved antibacterial activity is in NVB302, which is currently under clinical trials ${ }^{12,124}$.

In my study, in order to improve the druggability of MU1140, I modeled and investigated hybrid antibiotic peptides combining notable features of MU1140 and the EBOV delta peptide. In terms of membrane insertion and pore formation, the EBOV deltapeptide shares both structural and functional similarities with many other antimicrobial peptides $^{13,145}$. The abundance of basic and aromatic amino acids and the disulfide crosslink at the C-terminal makes this peptide a membrane pore former. Addition of more positively charged amino acids improves negatively charged membrane interaction and enhances the solubility due to increased polarity. In addition, it can have a higher production yield when the C-terminal AviCys ring is replaced with the conserved delta peptide anchorage. It could be less prone to proteolytic degradation and oxidation because the presence of a disulfide cross-link can improve the stability, as observed in other proteins and peptides ${ }^{101,146}$.

To create hybrids, I used the front part of MU1140 and the back part of the EBOV delta peptide, as seen in Fig. 5.1. Specifically, I selected the lipid-II binding segment 
composed of the N-terminus' first 13 amino acids of MU1140 and three different length sequences of amino acids from the C-terminal end of the EBOV delta peptide from positions 22-40, 25-40, and 29-40. I connected the MU1140 13 amino acid segment's Cterminus with the EBOV peptide segments' $\mathrm{N}$-terminus, thus producing three different peptide models. I generated an additional three variants by introducing a single-point mutation Glu33Arg. Hopefully, these peptides will have both the lipid II binding features from the first two rings of MU1140 and the pore-forming ability from the C-terminal amphipathic delta peptide. Using molecular dynamics simulations, I investigated these peptides to evaluate their druggability. Our study shows that the longest hybrid peptide containing residues 22-40 from the EBOV delta peptide with negatively charged Glu33 stands out among the other five with regard to the overall assessments of solubility, binding affinity with lipid II molecules, membrane insertion, and pore formation ability. Such a detailed study may be helpful in designing better antimicrobial drugs and unravel new pipelines of hybrid chemical entities with the combination of antibiotics and other poreforming viral and antimicrobial peptides. 


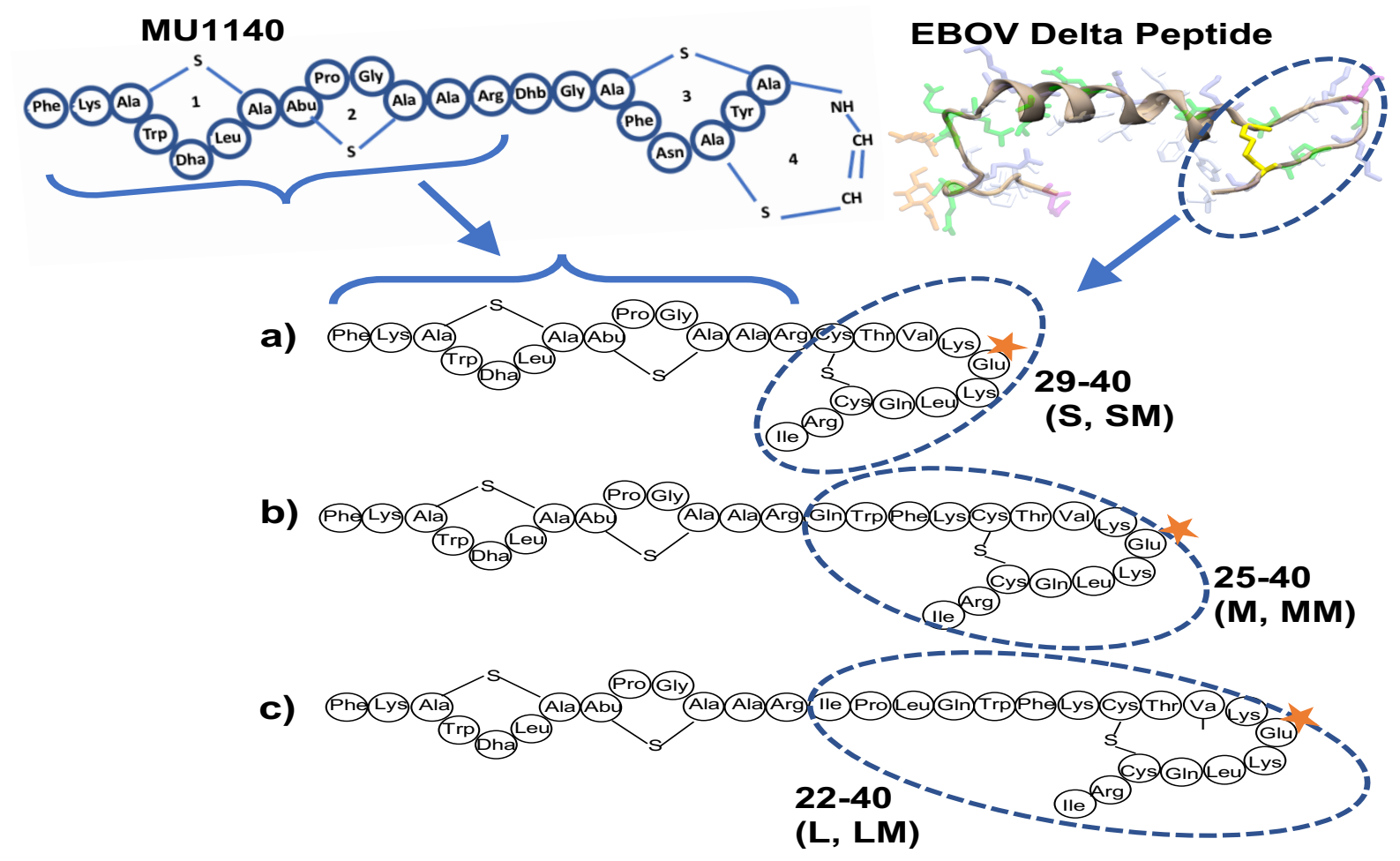

Figure 5.1 Hybrid model from MU1140 and the EBOV delta peptide with possible mutation. a) The shorter models S and SM. b) Medium models M and MM. c) Longer models L and LM. The mutated models SM, MM, and LM are from mutating Glu33 (orange star) with Arginine.

\subsection{Modeling the Hybrid Structure}

The structures of MU1140 and the EBOV delta peptide were taken from our previous studies $^{29,72}$. MU1140 has 21 amino acids, and the delta peptides contain 40 amino acids (Figure 1.1). To build the hybrid peptide, I included the first 13 amino acids from MU1140, which includes all the amino acids from the lipid II binding cage and the charged amino acid Arg13 essential for membrane interaction and water channel formation. From the EBOV delta peptide, I took three different amino acid segments of different lengths from the C-terminal end. The shorter model (S) includes the residues from 29-40 (Figure 5.1a), the medium model contains residues from 25-40 (Figure 5.1b), and the longer model 
(L) contains residues from 22-40 (Figure 5.1c) from the EBOV delta peptide. I also modeled the same peptides but with a single point mutation of negatively charged (at neutral $\mathrm{pH}$ ) Glu33 substituted with positively charged Arg33, named SM, MM, and LM, respectively. The wild-type MU1140 is denoted as WT. The total number of amino acids and their charges in all variants are shown in Table 5.1. All of the hybrid variants have a higher fraction of polar and charged residues than WT.

\begin{tabular}{|c|c|c|c|c|}
\hline Variants & $\begin{array}{c}\text { Total } \\
\text { Residue } \\
\text { No. }\end{array}$ & $\begin{array}{c}\text { Polar } \\
\text { Residue } \\
\%\end{array}$ & $\begin{array}{c}\text { Charged } \\
\text { Residue } \%\end{array}$ & $\begin{array}{c}\text { Total } \\
\text { Charge }\end{array}$ \\
\hline WT & 21 & 43 & 10 & 2 \\
\hline L & 32 & 44 & 22 & 5 \\
\hline LM & 32 & 44 & 22 & 7 \\
\hline M & 29 & 48 & 24 & 5 \\
\hline MM & 29 & 48 & 24 & 7 \\
\hline S & 25 & 48 & 24 & 4 \\
\hline SM & 25 & 48 & 24 & 6 \\
\hline
\end{tabular}

Table 5.1 Total number of residues and fraction of polar and charged residues of modeled structures

\subsection{Solubility Assessments}

Aqueous solubility of a drug is a major factor determining its bioavailability, a key to its effectiveness ${ }^{147-148}$. Poor solubility is one of the most common obstacles that prevent many newly discovered chemical entities to reach the market ${ }^{149-150}$. Drug solubility depends on various factors such as the polarity of the drug and the solvent, size of the drug, temperature, and $\mathrm{pH}$ of the solvent ${ }^{151-152}$. Though hydrophilicity influences the 
disaggregation and dissolution of drugs in solution, a soluble polar molecule may lack lipophilicity for drug permeation, and also suffer screening due to ionic charges in the solution ${ }^{153}$. Hence, pharmaceutical research focuses on modeling and optimizing novel amphipathic agents with a proper fraction of hydrophilicity and hydrophobicity, or combinations with functionalized groups or carriers for effective drug delivery ${ }^{148,}{ }^{154-155}$.

To examine our modeled structures' solution behavior, I performed molecular dynamics simulations for 500ns for each peptide in an explicit water system. Figure 5.2a, shows the initial (left of arrow) and final snapshots of the MD trajectories. The blue and red spheres represent the $\mathrm{N}$ and $\mathrm{C}$-terminal carbon atoms. All peptides collapse to globular structures, and continue to expose their polar (blue and green licorice structures) and charged residues to the water. To investigate the size and the flexibility of the peptides, I calculated the radius of gyration (RGYR) from the last $200 \mathrm{~ns}$ of the MD production run using a VMD plugin and display the results in a box plot in Figure 5.2b. The box plot depicts the median (center line), mean (blue star), and the fluctuations of RGYR. The average RGYR is the highest for the LM peptide and is the least for the WT, M, and S variants. LM also has the highest RGYR fluctuations. Interestingly, the $\mathrm{L}$ variant has a significantly smaller average RGYR than LM, and the L variant has the smallest RGYR fluctuation of all variants. All the variants with Glu33 have smaller average RGYR and smaller fluctuations than the mutated variants with Arg33. The conformational flexibility in monomeric form can be linked to a peptide's solubility: the more flexible the peptide, the less likely it is to form a hydrophobic cluster or aggregate. However, other structural and dynamical factors such as intra-peptide hydrogen-bonding, surface area-to-size ratio, fluctuation in the hydration layer, and the abundance of charged residues may govern the 
solubility ${ }^{148,156-157}$.
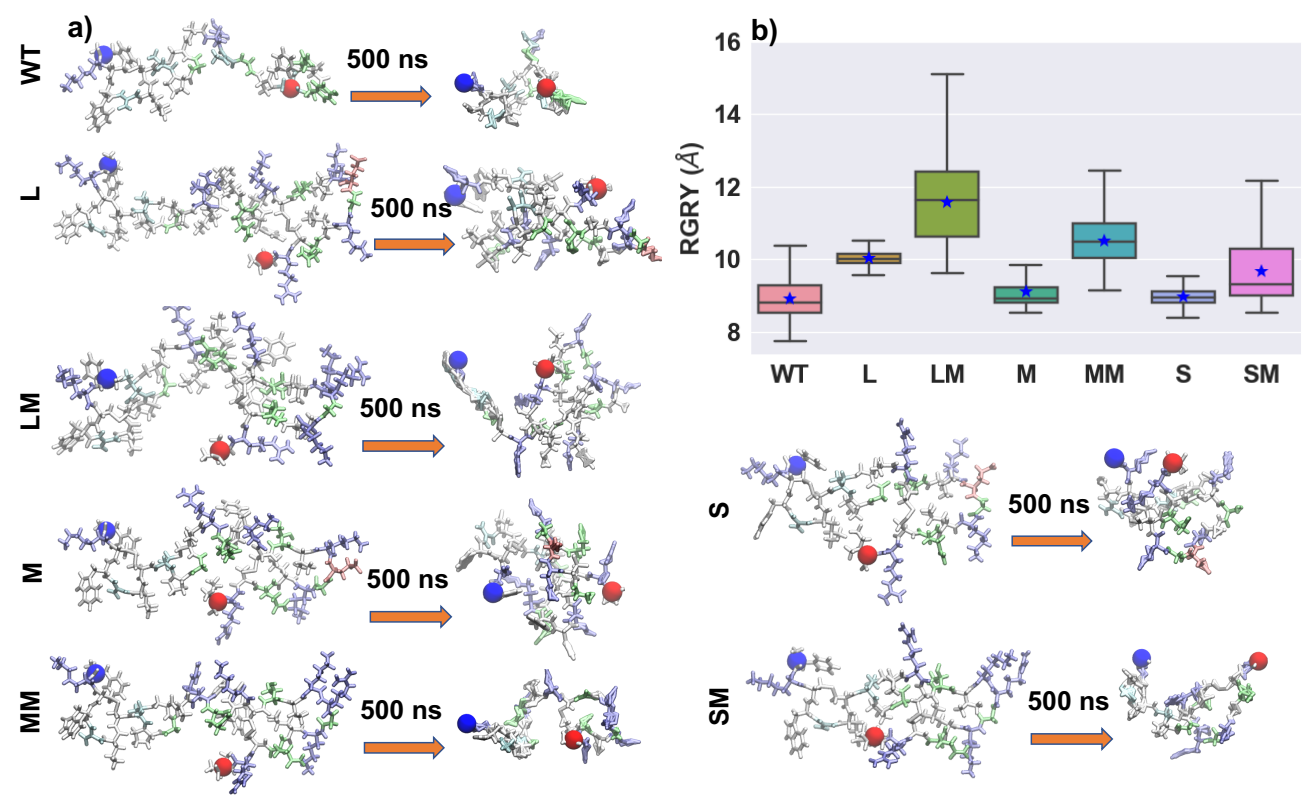

Figure 5.2 a) Snapshots of peptides form the initial (left of arrow) and final frames of 500 ns MD trajectories. The blue and red spheres represent the $\mathrm{N}$ and $\mathrm{C}$-terminal carbon atoms. The blue and green licorice structures represent the charged and polar amino acids while the white color represents the hydrophobic amino acids. b) Box plot of the average radius of gyration (RGYR) form the last $200 \mathrm{~ns}$ of the MD production run. The blue star represents the mean value.

From the last $200 \mathrm{~ns}$ of the simulation, I also calculated (Table 5.2) several parameters related to the peptide variants' solubility, including the number of hydrogen bonds (H-bonds) with water and the solvent accessible surface area (SASA) using the VMD plugin. I also calculated the average number of water molecules within $2.5 \AA$ of the peptide, representing the water molecules in the first hydration layer, which is responsible for the interactions with the peptides. In Fig. 5.3, I plotted the data for the hydrogen bonds, average number of water molecules, along with the number fluctuation (Hy-fluctuations), 
and the SASA. The plot has a relative vertical scale, with the largest value for each category normalized to $100 \%$ for better comparison. Table 5.2 shows that the H-bonds for the first three peptides WT, L and LM have a maximum hydrogen bonding of around 38 (converted to $100 \%$ in Fig. 5.3), suggesting better solubility for these peptides. The LM peptides have the a much higher water fluctuation in the first hydration layer than the other two, and the WT has the smallest water fluctuations of all the peptides. The WT also has the smallest hydrophilic SASA and smallest hydrophobic SASA of all peptides due to its short length (Table 5.1) and compact configuration (Fig. 5.2). The hydrophilic SASA is maximum for the MM peptide. Based on comparison of the hydrogen bonds with water and the hydrophilic SASA, it is expected that the solubility of L, LM models is comparable to WT. Also, more hydrophilicity or the number of charged residues might lead to a better solubility.

\begin{tabular}{|c|c|c|c|c|c|}
\hline Variants & $\begin{array}{c}\text { No. H-bonds } \\
\text { with water }\end{array}$ & $\begin{array}{c}\text { Hydration } \\
\text { Fluctuation }\end{array}$ & $\begin{array}{c}\text { RGYR } \\
(\AA)\end{array}$ & $\begin{array}{c}\text { Hydrophilic } \\
\text { SASA } \\
\left(\AA^{2}\right)\end{array}$ & $\begin{array}{c}\text { Hydrophobic } \\
\text { SASA } \\
\left(\AA^{2}\right)\end{array}$ \\
\hline WT & 38.4 & 6.2 & 8.9 & 412.6 & 493.7 \\
\hline L & 38.2 & 9.1 & 10.0 & 553.4 & 694.3 \\
\hline LM & 38.5 & 15.3 & 11.6 & 604.8 & 804.5 \\
\hline M & 35.4 & 10.5 & 9.1 & 526.6 & 632.3 \\
\hline MM & 36.9 & 10.1 & 10.5 & 647.8 & 647.4 \\
\hline S & 29.8 & 13.2 & 9.0 & 489.1 & 564.4 \\
\hline SM & 31.1 & 12.7 & 9.7 & 563.4 & 535.2 \\
\hline
\end{tabular}

Table 5.2 Various parameters measured from averaging over the last $200 \mathrm{~ns}$ of the MD production run of the WT and hybrid variants. 


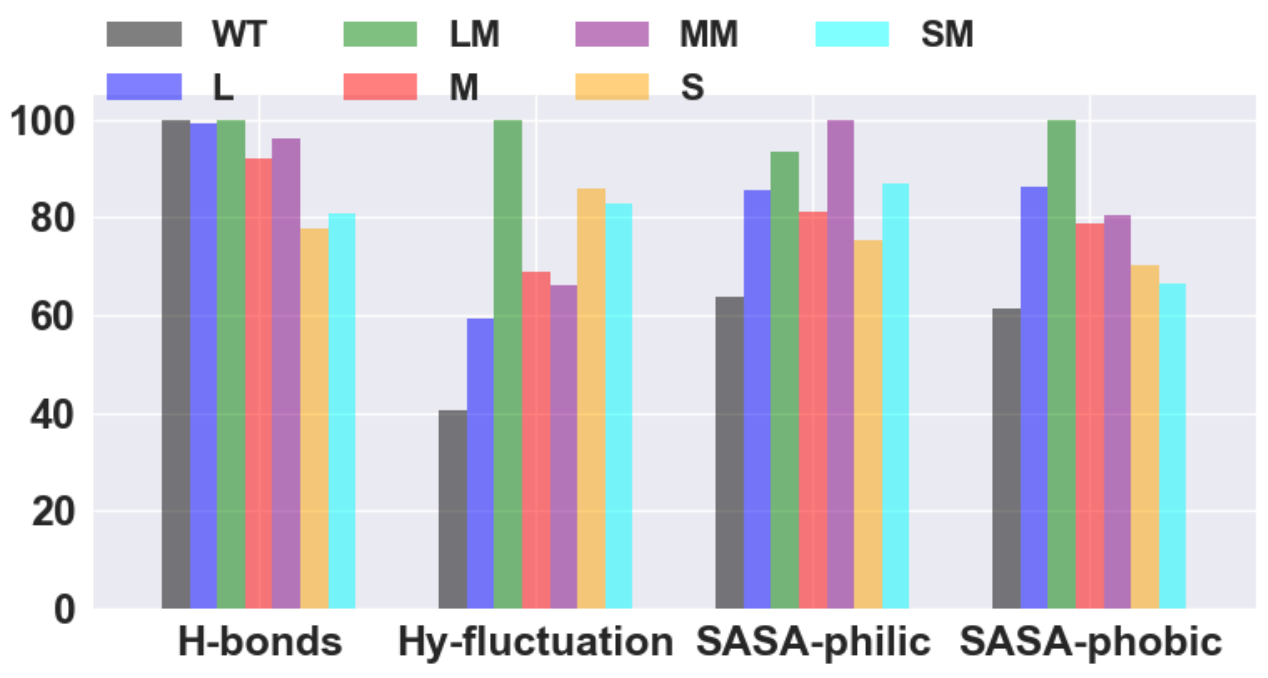

Figure 5.3 Normalized plot of various parameters from Fig. 5.2: H-bonds (number of hydrogen bonds of peptide with water molecules), Hy-fluctuation (fluctuation of water molecules in first hydration layer of peptides, SASA-philic (SASA of hydrophilic amino acids), and SASA-phobic (SASA of hydrophobic amino acids).

Accurately predicting the solubility of a peptide using MD observables is a complicated task and a more rigorous statistical and thermodynamic approach is needed to predict the solubility of peptides, especially the lanthipeptides composed of modified amino acids and sulfur bonds. Still, these hybrid peptides possess increased hydrophilicity and hydrophobicity than the WT, and this may lead to improved druggability of the peptides. 


\subsection{Lipid II Interaction}

Binding to the lipid II molecule and hindering its function in cell wall biosynthesis is a major mechanism of action for lantibiotics. Notably, the shorter lantibiotics like gallidermin have strong binding affinity with lipid $\mathrm{II}^{63}$. In addition to the lipid II binding moiety, the C-terminal tail of the peptide plays a role in binding and sequestering lipid II into large lipid II-lantibiotic clusters ${ }^{143}$.

To investigate the peptide interactions with the lipid II molecule, I prepared peptide-membrane systems for the six variants by placing them on the bacterial membrane surface, along with a lipid II molecule (Figure 5.4a). For each system, I performed $1 \mu$ s of MD simulation. 

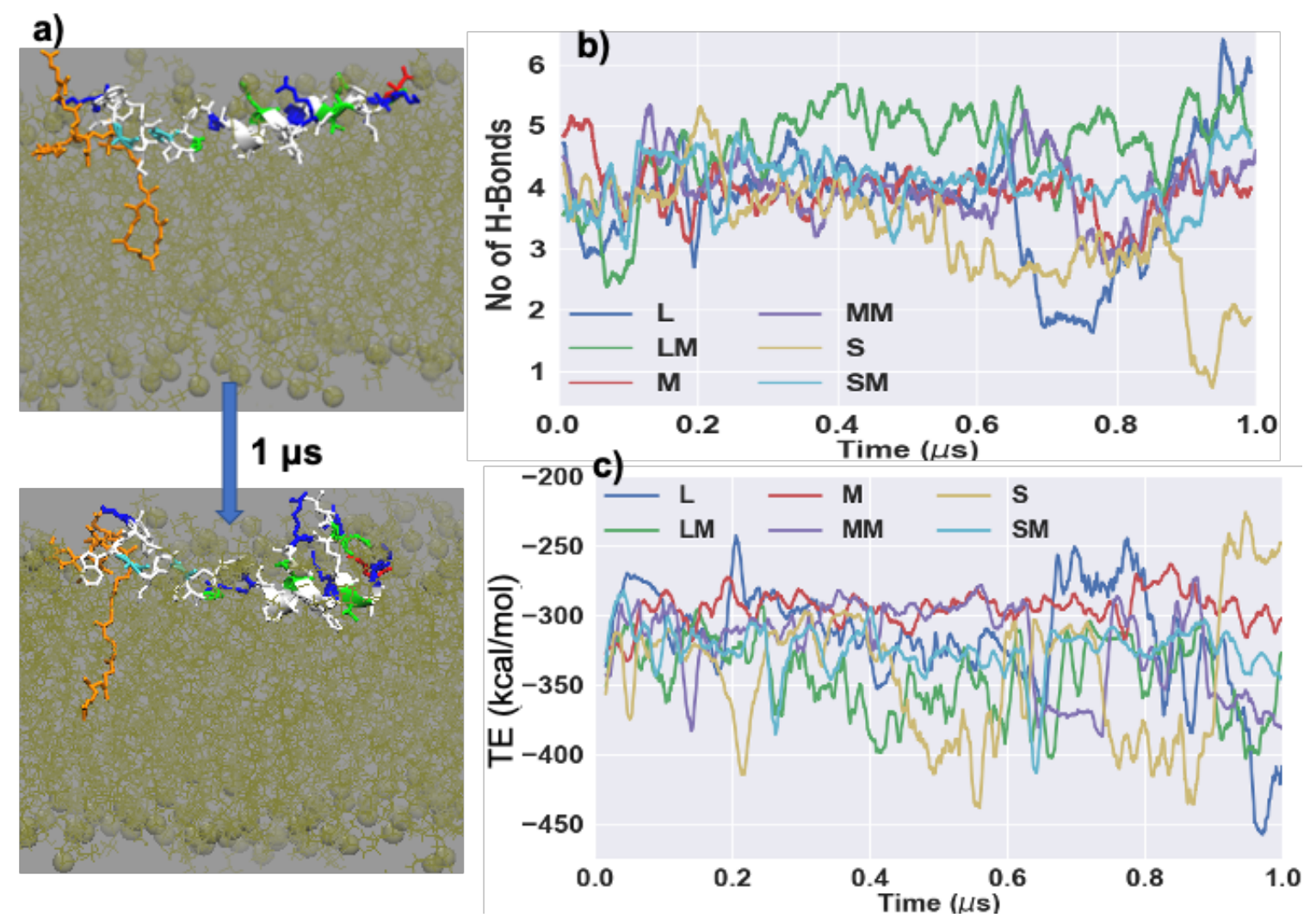

Figure 5.4 a) Snapshot of L variant initially placed at the surface of the membrane, and after $1 \mu \mathrm{s}$ of MD simulation. The brown molecules are lipids in the membrane and the brown spheres are phosphate atoms of lipids in the membrane surface. For all six variants, the time evolution of: (b) number of hydrogen bonds, and (c) interaction energies between the peptide and lipid II molecules.

Figure $5.4 \mathrm{~b}$ shows the time evolution of the number of hydrogen bonds between each of the variant peptides with lipid II molecules. Initially, the L model forms approximately four hydrogen bonds that changes to approximately six at the end of $1 \mu \mathrm{s}$. The hydrogen bonding in LM is stable throughout the simulation with approximately five hydrogen bonds. The $\mathrm{S}$ variants shows the weakest binding to the lipid II molecule with the least number of hydrogen bonds. Based on the hydrogen bonding, L and LM models appear to have a better lipid II binding affinity than WT variant, which forms 
approximately four hydrogen bonds with lipid II on the membrane surface in $500 \mathrm{~ns}$ simulation time scale (Figure 4.2c) ) $^{72}$. To compare the interactions for the variant peptides, I plotted the total interaction energy (electrostatic + van der Waals) calculated using the VMD module in Figure 5.4c. The interaction energy also shows the same pattern as the hydrogen bonding, with higher number of hydrogen bonds resulting in lower, stronger energy of interaction. The $\mathrm{L}$ variant shows a better binding with minimum energy and the $\mathrm{S}$ variant with highest energy at the end of $1 \mu$ s. Compared to the other variants, LM shows better overall interactions with lipid II, whereas $\mathrm{M}$ has the least fluctuations in the interaction with the lipid II molecule. The largest number of hydrogen bonds between lipid II molecules and the peptides are made with five mutacin amino acids that are common to all of the variants. In Fig. 5.5, I plot the number of hydrogen bonds to each of these amino acids, averaged over the last $300 \mathrm{~ns}$ of the MD simulations. The backbone nitrogen atoms of these residues play a dominant role in the hydrogen bonding. 


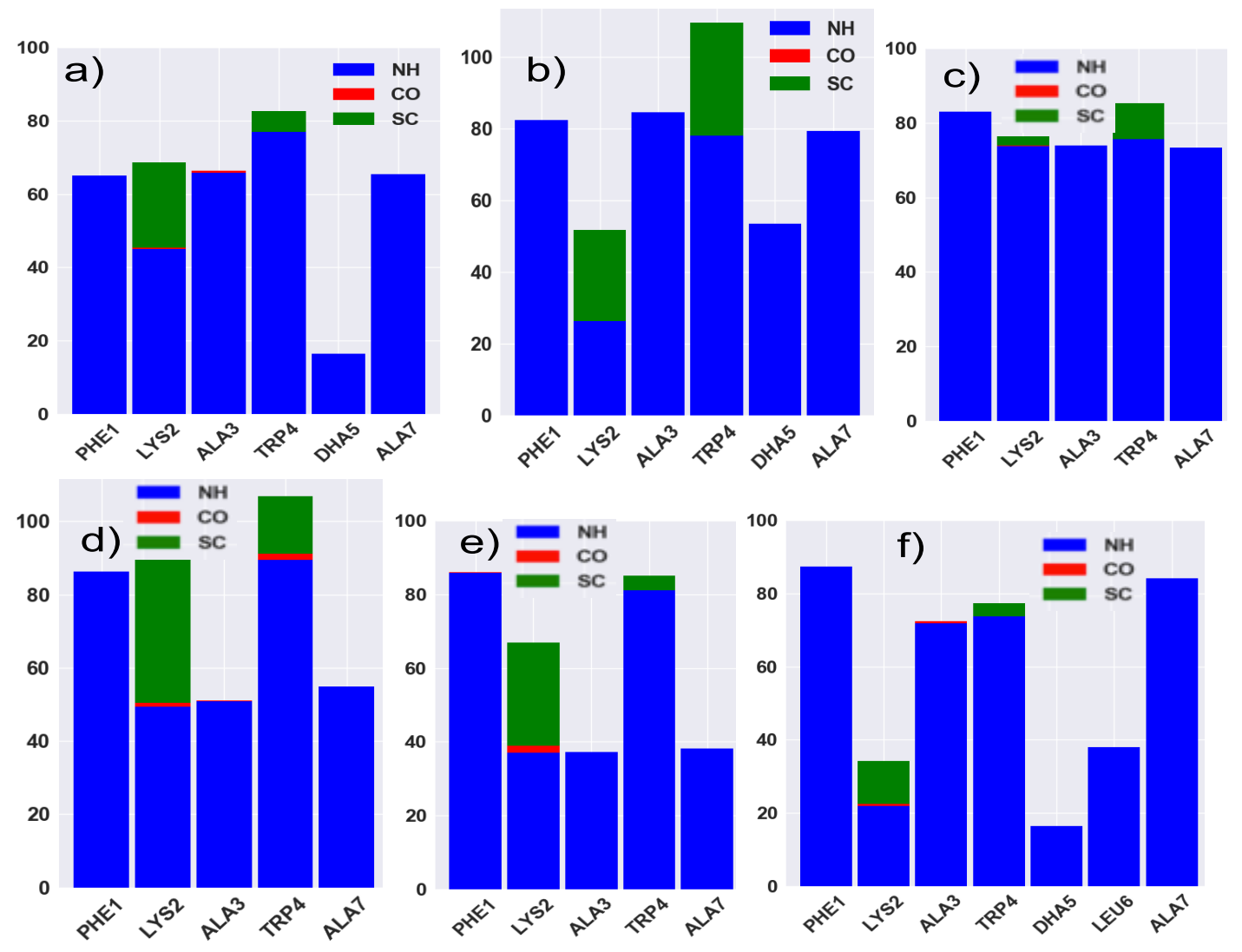

Figure 5.5 Percentage occupancy of hydrogen bonds between peptide amino acids and lipid II, averaged over the last 300 ns of $1 \mu$ s MD run for each variant: (a) L, (b) LM, (c) M, (d) MM, (e) S, (f) SM.

\subsection{Membrane Insertions}

In addition to the lipid II binding, other properties of the peptides such as membrane insertion, water pore formation, and disruption of the bacterial membrane are needed for the lantibiotics to possess dual modes of action. Therefore, the designed peptide models are desired to have a better or comparable membrane insertion profile compared to the WT. Insertion of a peptide using regular MD computations can require a time that is too long to be feasible. To quantify the insertion profile energetically, I performed steered molecular dynamics (SMD) computations and pulled the backbone carbon-alpha atoms of the residues Glu33, Lys34, and Leu35 (numbering based upon their positions in the Ebola delta 
peptide) of the peptides through the bacterial membrane bilayer at a constant velocity of $0.5 \AA / \mathrm{ns}$. For the mutated variants, Arg was substituted for Glu. Figure 5.6a displays the initial and final configuration of a peptide pulled through a membrane.

I calculated the magnitude of the pulling force along the negative $\mathrm{z}$-axis and plotted it in Figure 5.6b. The z-coordinate represents the center-of-mass of the backbone carbon atoms from the three residues that are pulled. The membrane spans a region from $-20 \AA$ (lower, inner leaflet) to $+20 \AA$ upper, outer leaflet). The graph shows that it requires an increasing force to pull the peptide variants through the membrane until the three pulled amino acids cross out of the lower leaflet of the membrane.
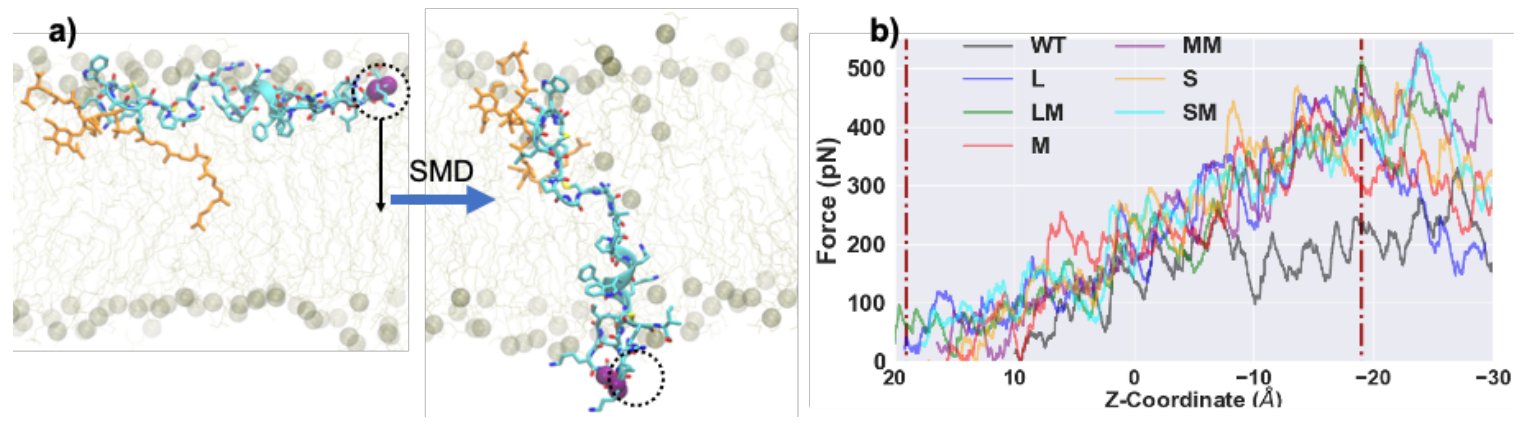

Figure 5.6 a) Pictorial representation of the SMD constant velocity pulling through the membrane of backbone carbon atoms of three amino acids at the tail of variant L. b) Force required to pull at constant velocity across the z-coordinate of the membrane. The two dotted vertical lines define the upper and lower boundaries of the lipid bilayer.

The trajectory of conformations during the SMD pulling of the peptides through the membrane allow further investigations by calculating the potential of mean force (PMF) using MD umbrella sampling and free energy profiles. For the umbrella sampling, I took 25 different conformations having the center-of-mass of the carbon atoms of the three pulled residues from $z=19 \AA$ to $z=-29 \AA$, giving 25 windows separated by $2 \AA$. For each window, I performed MD sampling simulations of $10 \mathrm{~ns}$. I used the WHAM tool to 
sample free energy values from the MD trajectories across various windows and plot the results in Figure 5.7. The graph shows that WT and M insertion are the most energetically favorable compared to other variants, followed by the L variant. The plot shows that the free energy is the most unfavorable for LM, MM, and S insertion into the membrane.

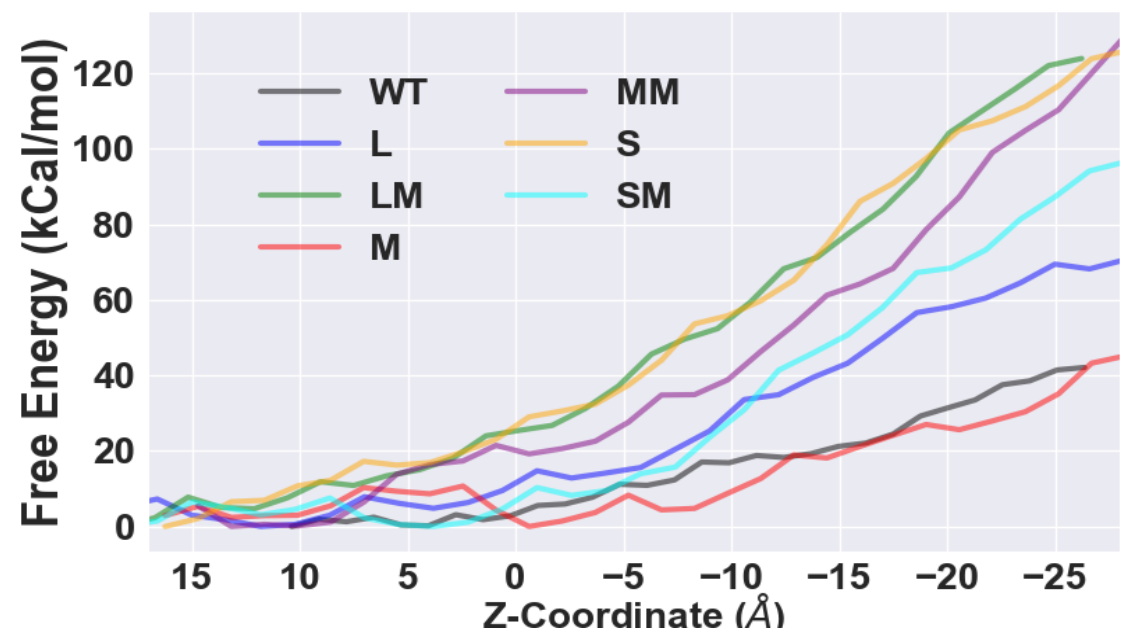

Figure 5.7 Free energy of pulling of peptides across the membrane.

In my previous work, I reported that despite having a significantly shorter chain compared to nisin, WT (MU1140) could span the transmembrane region and create a channel for water permeation ${ }^{72}$. To study the degree of spanning across the membrane and water channel formation of the six new variants, I placed them across the membrane along with a lipid II molecule (Figure 5.8a). I placed the N-terminal of a variant at the upper leaflet and the C-terminal at the lower leaflet of the membrane. From $1 \mu$ s MD simulations for each variant, I observed water permeation across the membrane, sliding along the peptides for all models. Figure 5.8a shows the initial and final snapshots of the $1 \mu \mathrm{s}$ trajectory for L. To quantify the water insertion behavior for each peptide, I located the 
average position of all water molecules along the z-axis within $5 \AA$ of the peptide from the last $300 \mathrm{~ns}$ of the trajectory. In Fig. 5.8b, I plot the probability density profile for water molecules. For the shorter models $\mathrm{S}$ and $\mathrm{SM}$, the bottleneck region is found to be around $\mathrm{z}$ $=10 \AA$, and for all others, it is around $\mathrm{z}=5 \AA$. The non-zero density profile suggests that all of these peptides are capable of forming an effective water channel. The expanded region in the inset shows that the $\mathrm{L}$ peptide is the most efficient at the bottleneck.

a)

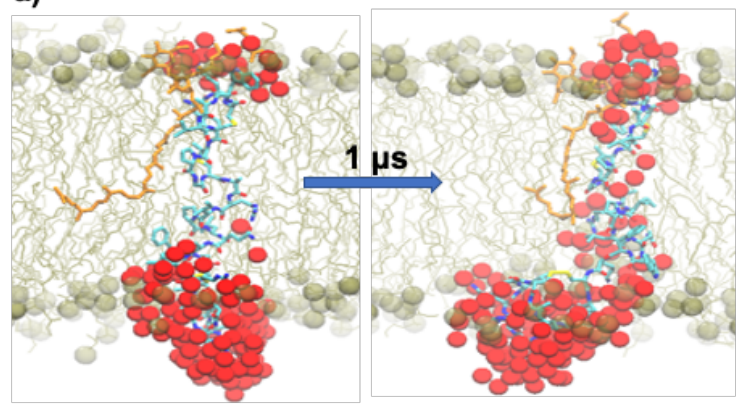

b)

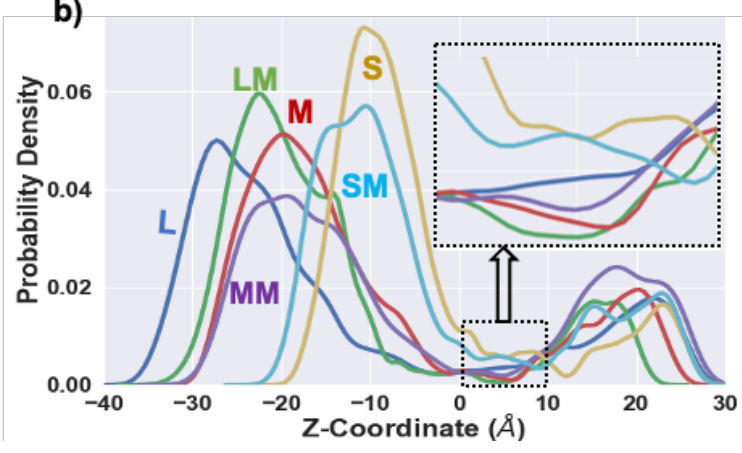

Figure 5.8. Water transport through the membrane. a) Initial and final snapshots of the $\mathrm{L}$ peptide place across the membrane. The red spheres denote the water molecules within $5 \AA$ of the peptide. b) Probability density, averaged over the last $300 \mathrm{~ns}$ of the MD simulation, of water molecules within $5 \AA$ of peptides placed across the membrane.

Figure $5.8 \mathrm{~b}$ also shows the degree of water 'funneling' by peptides outside the lipid bilayer. The extent of water funneling is represented by the left and right peaks of the water probability densities in the graph. Though L and LM have the same number of residues, the L model funneling extends more than the LM model, and other shorter peptides have correspondingly shorter funneling profiles. More polar residues in the C-terminal account for the higher density peak on the left side (lower layer) than on the right side (upper layer).

\subsection{Membrane-Pore Formation}

As I observed the $\mathrm{L}$ and $\mathrm{M}$ models are energetically favorable for membrane 
insertion, I was interested to see if they can form oligomeric membrane pores, similar to Nisin and Mutacin as reported in recent studies ${ }^{64,72}$. Using a custom script, I modeled the pentameric pores for both variants by placing the corresponding monomers symmetrically with an average initial radius of approximately $6 \AA$. The pentameric oligomer was chosen based on the pentameric pore formation by the Ebola virus delta peptide ${ }^{29}$. I inserted the pore model into the bacterial membrane and ran the MD simulation for $500 \mathrm{~ns}$. Figure 5.9a and Figure 5.9b show the initial and final snapshots of the MD trajectories for the pores of $\mathrm{L}$ and $\mathrm{M}$ peptides. I observed that the $\mathrm{L}$ model's $\mathrm{C}$-terminal constitutes transmembrane alpha-helix, while the M model mostly has a loop or disordered structure. Figure 5.9c and Figure $5.9 \mathrm{~d}$ show that both pore models are effective at water transport across the membrane. I also calculated the water density profile using density profile tools ${ }^{99}$. Each density profile is a one-dimensional density calculated along the pore's z-axis and averaged over the last $300 \mathrm{~ns}$ of the MD trajectory. The graph in Figure 5.9e represents the density of water in terms of the number of water molecules $/ \AA^{3}$. This plot also demonstrates that both models can form equally efficient water channels across the bacterial membrane. 

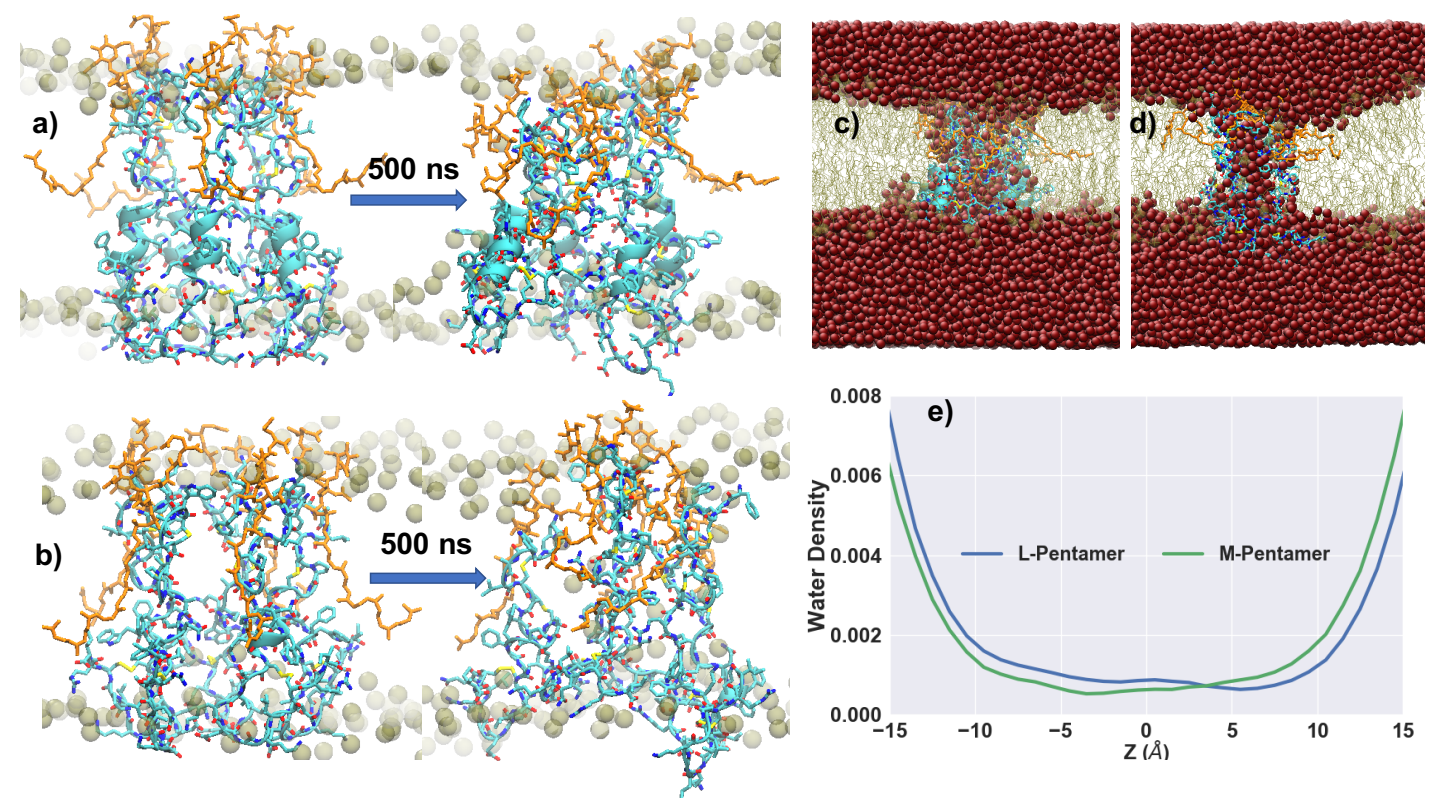

Figure 5.9 Initial and final snapshot of MD trajectories of pentamer pore of L (a) and M (b). Water transport across the membrane by pores of L (c) and M (d) model. e) Number of water molecules/Å3 projected along z-axis within the membrane region of $\mathrm{L}$ and $\mathrm{M}$ pentameric pores.

In Figure 5.10, I have quantified the size and stability of pores from the MD trajectories using HOLE program ${ }^{95}$. This program computes the volumetric map and transverse radius of pores based on the van der Waals radius of the peptide atoms. Figure 5.10a and Figure $5.10 \mathrm{~b}$ are the $3 \mathrm{D}$ visualization pore size for initial and final frames of $\mathrm{L}$ and M models from 500 ns production run. Sections that are too narrow to pass any water molecules would be colored red, green if they allow one water molecule to pass, and blue if they are wide enough to pass two or more water molecules. I observed two different bottlenecked regions; the $\mathrm{L}$ model has narrower restriction at the $\mathrm{N}$-terminal region while $\mathrm{M}$ has that around the $\mathrm{C}$-terminal region. In Figure 5.10c displayed the pore radius along the channels' z-axis during the final $300 \mathrm{~ns}$ along with the standard deviation. The midpoint of the membrane is chosen to define $\mathrm{z}=0$. In both pores, the average radius of the 
pores is higher than $2.5 \AA$, giving a diameter greater than $5 \AA$, which is significantly larger than the diameter of a water molecule, $2.8 \AA$. The $\mathrm{L}$ model has a minimum average radius around $\mathrm{z}=10 \AA$, while the $\mathrm{M}$ model has a wider minimal range from $\mathrm{z}=-5$ to $\mathrm{z}=5 \AA$. $\mathrm{L}$ model has a narrower deviation below $\mathrm{z}=10 \AA$, indicating that the $\mathrm{C}$-terminal helical structure contributes to forming stable and smooth membrane pore. Figure 5.10d displays the time evolution of root mean square deviation (RMSD) for $500 \mathrm{~ns}$ MD run with reference to initial model. The $\mathrm{L}$ model changes its configuration slowly, and after $350 \mathrm{~ns}$, it becomes stable. While there is an abrupt change in the M model configuration during the first $100 \mathrm{~ns}$ of MD run, and it becomes stable after that. At the end of $500 \mathrm{~ns}$, the L model's deviation is slightly lower, showing that it retains a more symmetric initial configuration than the M model.

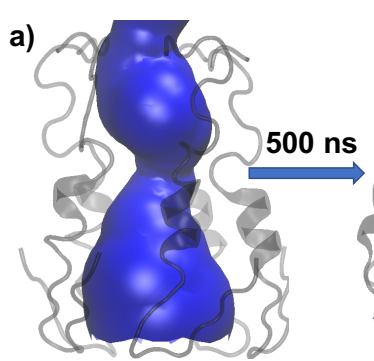

c)
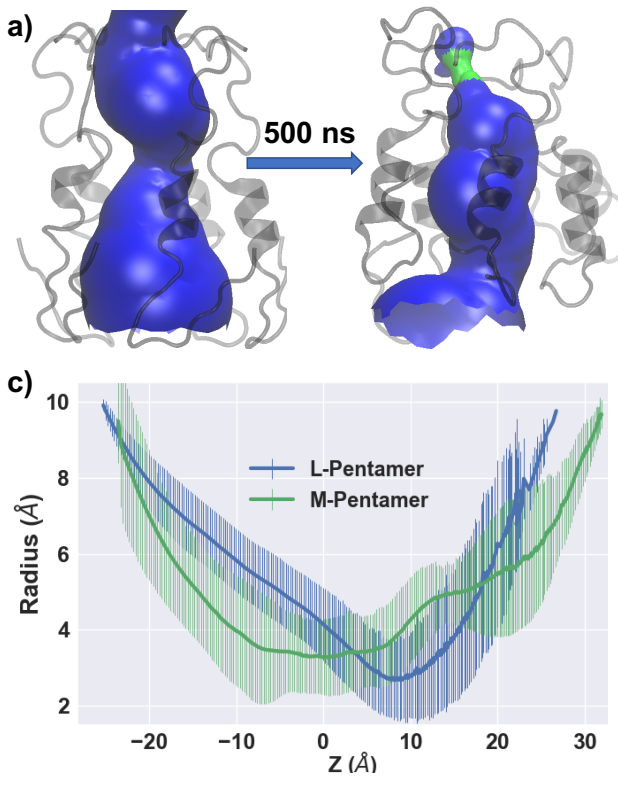

b)
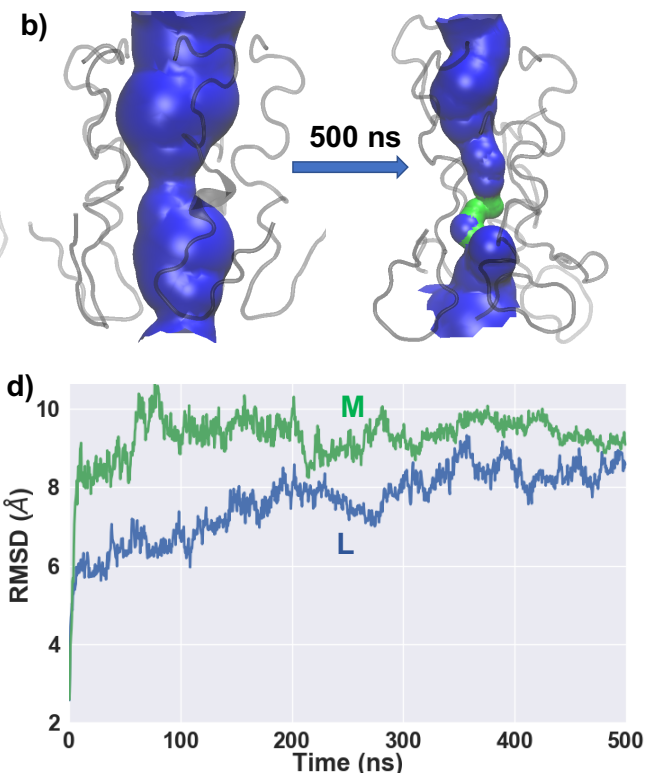

Figure 5.10 Volumetric map of pentameric pores of L (a) and M(b) models. c) Average radius of pores from last 200 ns. d) Time evolution of root mean square deviation of pore structures of $\mathrm{L}$ and $\mathrm{M}$ models. 


\section{CONCLUSIONS}

The EBOV delta peptide is a viroporin peptide that forms homo-oligomeric pores in the membrane. In this work, I modeled various delta peptide oligomeric pores of different numbers of peptides and systematically explored the pore stability, ion selectivity, and the role of the peptide disulfide bond using all-atom molecular dynamics simulations in an explicit lipid bilayer. I considered tetrameric, pentameric, and hexameric peptide assemblies of the EBOV delta peptide for membrane pore formation. Molecular dynamics simulations showed that the pore formed by the tetrameric assembly of the peptide is not stable. For the simulated timescales I studied, both pentameric and hexameric assemblies formed stable, ion-permeable membrane pores that selectively permeate water and chloride ions across the lipid bilayer. The pentameric pore is slightly more stable than the hexameric pore. Compared to the other oligomeric assemblies, the pentameric assembly allows a tighter packing of the adjoining helices, providing stronger van der Waals interactions as well as the two H-bonds that stabilize the pore. These interactions are weaker in the tetramer and hexamer. The pore radius, ion density and flux profiles show that the EBOV delta peptide pores may have directional selectivity for permeating chloride ions. In addition, our results show that the conserved disulfide-bond formation between Cys-29 and Cys-38 in a delta peptide is essential for pore stability and ion flux.

Overall, our computational study suggests that: (1) the EBOV delta peptide likely forms stable pentameric pores in the membrane bilayer, (2) the viroporin is selective in allowing a passage of chloride ions but prevents passage of sodium ions, and (3) the conserved intra-peptide disulfide-bond is essential for a pore's stability as well as for ion flux. These findings lead to future investigations to study the role of membrane 
composition ${ }^{158}$ and peptide concentration on pore formation as well as the energetic factors governing ion selectivity through the pores. Further studies with multiple replicas of various constructs as well as biophysical analyses are needed to ascertain the exact nature of the oligomeric assembly of delta-peptide in the membrane. Detailed understanding of the mechanisms of pore formation by delta peptide may lead to the design and development of targets for pore modulators and disruptors, and thus the ability to control virus propagation.

Another membrane-pore forming peptide I investigated is the antimicrobial peptide MU1140. The ability of MU1140 to bind to the lipid II pyrophosphate moiety in a membrane and abduct it from the septum of division, therefore obstructing bacterial cell wall synthesis, makes it a promising antimicrobial candidate for antibiotic-resistant pathogens.

The bacterial-killing mechanisms of certain antimicrobial peptides can be complex and involve more than a single mode of action. For example, it can include interference of the lipid II function and membrane pore formation or membrane disruption. Interestingly, it has been suggested that the lantibiotic spectrum of activity and potency is not necessarily related to the numbers of mechanisms involved. Bonelli et al. ${ }^{63}$ previously reported that epidermin and gallidermin are 10-20 times more potent against Lactococcus lactis than nisin, despite the missing pore formation capacity on that strain. In addition, it was previously shown that the [A12L] gallidermin variant is as active as wild-type gallidermin against Micrococcus flavus, despite losing its pore-forming activity. ${ }^{63}$

Peptides that have similar moieties and structural features as MU1140, including nisin, epidermin, and galidermin, have been shown to possess the dual functions in several 
bacterial strains. However, the molecular mechanisms of how MU1140 and its variants interact with lipid II or form the permeable membrane pores have only been inferred from the structural similarities with nisin and gallidermin. In this work, I explored the interactions of MU1140 with lipid II as well as the mechanism and sequence of membrane pore formation by the MU1140-lipid II complexes in a Gram-positive bacterial membrane using MD simulations. As with nisin, the anionic pyrophosphate moiety of lipid II provides the binding target for the thioether rings in MU1140. The formation of a stable MU1140lipid II complex highlights the lipid II trafficking mechanism of MU1140 suggested by experimental studies ${ }^{61,159-160}$.

Despite structural differences in the $C$-terminal tail as well as peptide length between nisin and MU1140, our results show that MU1140-lipid II complexes are able to form functional, water permeating membrane pores. Even a single chain of MU1140 complexed with lipid II is able to permeate water molecules across the membrane via a single-file water transport mechanism, which is commonly observed in nanoscale confinement such as carbon nanotubes and protein channels. The ordering of the water molecules in the single-file chain region as well as the diffusion behavior is similar to those observed in other biological water channels such as gramicidin A. Multiple complexes of MU1140-lipid II in the membrane showed enhanced permeability for the water molecules, as well as noticeable membrane distortion and lipid relocation towards the central region of the lipid bilayer. The results suggest that a higher concentration of MU1140 assembly in the membrane can cause significant disruption of the bacterial membrane. These investigations, for the first time, provide an atomistic level insight into a novel mode of action for MU1140. Further biophysical investigations with different variants of MU1140, 
lipid compositions, and multimeric assemblies can provide important insights for the rational design of optimized peptide variants with improved antimicrobial properties.

Ribosomally synthesized and post-translationally modified lantibiotics are potent antimicrobial compounds that exert their action against Gram-positive bacteria via a dual model of action, including having a unique binding site to lipid II molecules. Studies have found that variants of some of these lantibiotics are effective against resistant strains such

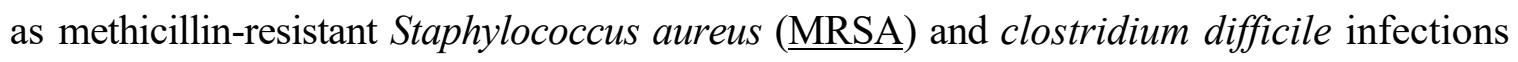
$(\mathrm{CDI})^{53,161}$. I investigated five lantibiotic variants: nisin, MU1140, gallidermin, NAI107 and NVB302. Using atomic-scale molecular dynamics computations, I examined the ability of these five different peptides to participate in two different anti-bacterial mechanisms: facilitate water transport across a membrane, and to form bonds to lipid II molecules.

My study shows that all of these five lantibiotics are able to form water transport channels across the membrane, but the efficiency of the channels differs widely. Nisin forms the most effective membrane channel that passes the largest number of water molecules per unit time. Though nisin provides a reasonably large number of different trajectories through the membrane for water transport, it is not the widest range of trajectories of the peptides. However, nisin's water trajectories have the fewest number of roadblock delay points along the length of the channel. This combination of a mid-level number of different trajectories, along with no significant delay points results in nisin being the most effective of the peptides in facilitating water transport across a model membrane. Therefore, a strategic placement of hydrophilic residues along the peptide sequence may facilitate water passage through the membrane, although this remains to be confirmed 
experimentally. However, this benefit from hydrophilic amino acids must be delicately balanced by the effect of hydrophilic amino acids on the ability for the peptide to insert into the hydrophobic membrane interior, which requires further investigation. I found that, though gallidermin and MU1140 have the same number of amino acids, the presence of Lys4 and Lys 13 in gallidermin prevents it from spanning the membrane and greatly diminishes gallidermin's ability to transport water. The shortest peptide, NVB302, facilitates a mid-level of water transport. NVB302 accomplishes this by pulling the membrane surfaces inward towards each other. This makes the membrane thinner and allows the short NVB302 to span the tapered membrane.

Though gallidermin was the least effective in facilitating water transport across the membrane, it is very effective at forming hydrogen bonds to lipid II molecules, along with MU1140. Therefore, gallidermin's ability to kill bacteria may better correlate to its strong binding to lipid II and impeding the critical functioning of lipid II in bacterial cell wall biosynthesis, rather than forming membrane pores. MU1140 is reasonably effective at both mechanisms. In contrast, nisin is especially effective in facilitating water transport, and is known to allow ion transport as a "true" pore-former but makes a relatively small number of hydrogen bonds with lipid II. The globular lantibiotics, NVB302 and NAI107, are similar to MU1140 in that they are moderately effective in both facilitating water transport and in forming bonds to lipid II molecules. These investigations provide atomistic insight to the mechanism of water channel formation and lipid II interaction of these five lantibiotic peptides. These studies provide insight that can guide the design of novel antibiotics. 
The dual modes of antimicrobial activity: 1) blockage of peptidoglycan synthesis by binding to and abduction of lipid II and 2) membrane pore formation ability, makes lantibiotics promising candidates for antibiotic-resistant pathogens. However, the poor solubility, low production yield, and susceptibility to degradation by digestive enzymes are major challenges encountered for oral administration and widespread uses of lantibiotics. In this work, I showed that by creating hybrid peptides composed of lantibiotic segments with the combination of an EBOV delta peptide segment, we can improve at least some of these challenges. The hybrid peptide models contain the lipid II binding cage of MU1140 and the conserved C-terminal transmembrane amphipathic helix from the EBOV delta peptide. From the molecular dynamic simulations, I assessed various factors which can determine the druggability of these models. I found that the longer models provide more charged amino acids and better hydrophilicity than WT, and hence might have better solubility. The overall lipid II binding affinity is best for the LM hybrid during our simulation time scales evaluated by the energy of interaction and hydrogen bonding with lipid II. On the other hand, SMD force and free energy analysis from MD umbrella sampling indicate that the membrane insertion profile is energetically favorable for $\mathrm{L}$ and $\mathrm{M}$ models. These $\mathrm{L}$ and $\mathrm{M}$ models form efficient pentameric membrane pores, though the pore from the $\mathrm{L}$ model is slightly more stable and smoother than from the M hybrid.

In summary, the L model has a better profile among the six hybrid variants, and its hydrophilicity, hydrophobicity, and lipid II binding profile are even better than MU1140. The $\mathrm{L}$ variant, having a amphipathic transmembrane helix, not only forms a more efficient membrane pore, it is also less likely to be degraded by proteolytic enzymes due to conserved disulfide bonds as observed in many peptide and proteins. Finally, the major 
advantage of this hybrid structure could be the high production yield due to the absence of an AviCys ring. Though experimental procedures should be performed to verify these computational results and speculations, this study proposed a new peptide scaffold for efficient therapeutics against antibiotic resistance via numerous permutations of lantibiotics and transmembrane peptides.

\section{OVERALL SUMMARY}

Membrane-permeabilizing peptides evolve for offensive or defensive purposes as viral peptides, host-defense peptides, venom, or toxins. Viral peptides function as a viroporin and form pores in the host cell in both bacterial and Eukaryotic membranes for the primary purpose of replicating and propagating viral particles ${ }^{100,} 162-163$. Structural and dynamical studies of such viroporins is essential for the development of antiviral drugs that could interfere with the pore functionality and hence the propagation of viruses ${ }^{18}$. Host-defense antibacterial and antiviral peptides disrupt the bacterial membrane and viral envelopes ${ }^{164-}$ 168. These antimicrobial peptides are a plausible candidate for the development of novel antibiotics. Discovery of novel antibiotics, especially those with novel mechanisms of action, is critical to mitigating the threat of antibiotic-resistance ${ }^{169}$. Antibiotics containing lanthionine, referred to as lantibiotics ${ }^{170}$, constitute a promising class of drugs to battle the threat of antibiotic resistance.

The Ebola virus (EBOV) is a filamentous lipid-enveloped virus that causes severe hemorrhagic fever with a high fatality rate in humans ${ }^{171}$. The EBOV encodes a glycoprotein that when cleaved, produces the delta peptide. Experimental evidence suggests that the delta peptide functions as a viroporin that enhances virus particle release 
through the host cell membrane ${ }^{20,30}$. However, the viroporin forming mechanism of the delta peptide is still not well understood. Guided by experimental information, I have computationally investigated the pore formation by different oligomers of the delta peptide. I have performed all-atom molecular dynamics (MD) simulations in an explicit membrane environment to investigate the pore-forming mechanism and stability of the pores. The results suggest that the delta peptide forms stable pentameric pores. In addition, the pore is selective with respect to chloride ions, and the disulfide bond formed between Cys-29 and Cys-38 in the C-terminal of the peptide is essential for the pore stabilization and ion permeation. This study provides helpful information on the pore-forming mechanism of filovirus delta peptides and such structural information can be important in designing and developing molecular modulators that target the delta peptide pore and disrupt the pathology of the Ebola virus.

Lantibiotics are ribosomally synthesized peptides that contain post-translationally modified unusual amino acids ${ }^{102,130}$. Lantibiotics are promising candidates to engage in the fight against resistant strains of bacteria due to their unique modes of action ${ }^{12}$. It is proposed that the antimicrobial activities of Lantibiotics are attributed to two modes of action: 1) vancomycin-like blockage of the peptidoglycan synthesis by binding to and abduction of lipid II and 2) membrane pore formation ${ }^{61,64,172-173}$. One such variants, Mutacin 1140 (MU1140) produced by Streptococcus mutans ${ }^{54}$ is a promising antimicrobial lanthipeptide and is effective against Gram-positive bacteria. I explored the potential mechanisms of Lipid II binding and membrane pore formation by performing molecular simulations of the MU1140-lipid II complex in the bacterial membrane. My investigation suggest that MU1140-lipid II complexes are able to form water permeating membrane pores. I find that 
a single chain of MU1140 complexed with lipid II in the transmembrane region can permeate water molecules across the membrane via a single-file water transport mechanism. The ordering of the water molecules in the single-file chain region as well as the diffusion behavior is similar to those observed in other biological water channels. Multiple complexes of MU1140-lipid II in the membrane showed enhanced permeability for the water molecules, as well as a noticeable membrane distortion and lipid relocation, suggesting that a higher concentration of MU1140 assembly in the membrane can cause significant disruption of the bacterial membrane.

In another study, I use atomic-scale molecular dynamics (MD) computational studies to compare both the lipid II binding ability and the membrane water channel formation mechanisms and efficacy of five different lantibiotics that are part of the focus of current antimicrobial research. They are nisin produced by Gram-positive bacteria Lactococcus lactis ${ }^{122}$, Mutacin 1140 (MU1140) produced by Streptococcus mutans ${ }^{54}$, gallidermin produced by Staphylococcus gallinarum $^{123}$, NVB302 isolated from Actinoplanes liguriae ${ }^{124}$, and NAI107, also known as microbisporicin or 107891, produced by the actinomycete Microbispora ${ }^{125}$. Where NVB302 and NAI107 are chemically modified semi-synthetic lantibiotics ${ }^{125-127}$. I find that all five of these peptides bind with the lipid II molecule, and also have the potential to form membrane water channels. However, the relative abilities vary dramatically. Among these, nisin is found to be the most effective at permeating water across the membrane. Compared to other variants, Gallidermin and MU1140 are found to bind more strongly to lipid II molecules. The shorter peptide NVB302 has the interesting ability to taper the membrane bilayer which can lead to membrane disruption. 
Lantibiotic Mutacin 1140 (MU1140) has shown a broad spectrum of activity against Gram-positive bacteria, including Clostridium difficile infection ${ }^{11,174}$. However, poor aqueous solubility, susceptibility to proteolytic degradation, and low production yield of lantibiotics are significant hindrances for their bioavailability, especially for oral administration and cost-effective commercial production ${ }^{139-140,143,175}$. The preferable way to address those challenges could be the hybridization of these peptides with other peptides to improve the overall drug profile ${ }^{134-136,138}$. In this work, I explored the hybridization of MU1140 and Ebola virus (EBOV) delta peptide to design the potential antimicrobial chemical entities. Our result suggests that among various hybrid variants the L model, constitute of 1-13 amino acids from MU1140 and 22-40 amino acids form EBOV delta peptide, shows better profile in term of solubility, binding with lipid II molecule, bacterial membrane insertion, and pore formation mechanism. These thermodynamical investigations could be useful for the design and development of potential antimicrobial entities by hybridizing lantibiotics with other pore-forming viral and antimicrobial peptides to address poor drugability of lantibiotics to mitigate the threat of resistant bacteria.

A future direction could be to design a ligand molecule that can bind with the Ebola virus pentameric pore and hinder the pore function. Among five variants that I investigated, the lantibiotic MU1140 is equally effective for lipid II binding and membrane channel formation. Hence, the future focus should be on investigating variants of MU1140 from single or multiple point mutations of amino acids. Insight into the lipid II binding, water-solubility, membrane insertion, and pore formation could help develop a novel molecule with optimized bactericidal properties. Among the hybrid variants that I investigated, L and LM variants' solubility and lipid II binding profiles were the best. On 
the other side, $\mathrm{M}$ has the lowest free energy for membrane insertion. Therefore, it is worthwhile to investigate the hybrid molecules having amino acids sequences between $\mathrm{L}$ and $\mathrm{M}$ models in the future. 


\section{References:}

1. Mitra, K.; Ubarretxena-Belandia, I.; Taguchi, T.; Warren, G.; Engelman, D. M., Modulation of the bilayer thickness of exocytic pathway membranes by membrane proteins rather than cholesterol. Proceedings of the National Academy of Sciences of the United States of America 2004, 101 (12), 4083-4088.

2. Spector, A. A.; Yorek, M. A., Membrane lipid composition and cellular function. $J$ Lipid Res 1985, 26 (9), 1015-35.

3. Goldfine, H., Bacterial membranes and lipid packing theory. J Lipid Res 1984, 25 (13), 1501-7.

4. van Meer, G.; Voelker, D. R.; Feigenson, G. W., Membrane lipids: where they are and how they behave. Nat Rev Mol Cell Biol 2008, 9 (2), 112-24.

5. Frolov, V. A.; Shnyrova, A. V.; Zimmerberg, J., Lipid polymorphisms and membrane shape. Cold Spring Harb Perspect Biol 2011, 3 (11), a004747.

6. Avci, F. G.; Akbulut, B. S.; Ozkirimli, E., Membrane Active Peptides and Their Biophysical Characterization. Biomolecules 2018, 8 (3).

7. Guha, S.; Ghimire, J.; Wu, E.; Wimley, W. C., Mechanistic Landscape of MembranePermeabilizing Peptides. Chem Rev 2019.

8. Lazzaro, B. P.; Zasloff, M.; Rolff, J., Antimicrobial peptides: Application informed by evolution. Science 2020, 368 (6490).

9. Jenssen, H.; Hamill, P.; Hancock, R. E., Peptide antimicrobial agents. Clin Microbiol Rev 2006, 19 (3), 491-511.

10. Seyfi, R.; Kahaki, F. A.; Ebrahimi, T.; Montazersaheb, S.; Eyvazi, S.; Babaeipour, V.; Tarhriz, V., Antimicrobial Peptides (AMPs): Roles, Functions and Mechanism of Action. International Journal of Peptide Research and Therapeutics 2019.

11. Kers, J. A.; Sharp, R. E.; Defusco, A. W.; Park, J. H.; Xu, J.; Pulse, M. E.; Weiss, W. J.; Handfield, M., Mutacin 1140 Lantibiotic Variants Are Efficacious Against Clostridium difficile Infection. Front Microbiol 2018, 9, 415.

12. Sandiford, S. K., Current developments in lantibiotic discovery for treating Clostridium difficile infection. Expert Opin Drug Discov 2019, 14 (1), 71-79.

13. Mahlapuu, M.; Hakansson, J.; Ringstad, L.; Bjorn, C., Antimicrobial Peptides: An Emerging Category of Therapeutic Agents. Front Cell Infect Microbiol 2016, 6, 194.

14. Luis Nieva, J.; Carrasco, L., Viroporins: Structures and Functions beyond Cell Membrane Permeabilization. Viruses 2015, 7 (10), 5169-71. 
15. Nieva, J. L.; Madan, V.; Carrasco, L., Viroporins: structure and biological functions. Nat Rev Microbiol 2012, 10 (8), 563-74.

16. Pokhrel, R.; Pavadai, E.; Gerstman, B. S.; Chapagain, P. P., Membrane pore formation and ion selectivity of the Ebola virus delta peptide. Phys Chem Chem Phys 2019.

17. Guha, S.; Melnik, L.; Garry, R. F.; Wimley, W. C., Ebola Virus Delta-Peptide Acts as an Enterotoxic Viroporin In Vivo. Biophysical Journal 2018, 114 (3, Supplement $1), 265 \mathrm{a}$.

18. Gervais, C.; Do, F.; Cantin, A.; Kukolj, G.; White, P. W.; Gauthier, A.; Vaillancourt, F. H., Development and validation of a high-throughput screening assay for the hepatitis C virus p7 viroporin. J Biomol Screen 2011, 16 (3), 363-9.

19. Guidotti, G.; Brambilla, L.; Rossi, D., Cell-Penetrating Peptides: From Basic Research to Clinics. Trends Pharmacol Sci 2017, 38 (4), 406-424.

20. He, J.; Melnik, L. I.; Komin, A.; Wiedman, G.; Fuselier, T.; Morris, C. F.; Starr, C. G.; Searson, P. C.; Gallaher, W. R.; Hristova, K.; Garry, R. F.; Wimley, W. C., Ebola Virus Delta Peptide is a Viroporin. J Virol 2017.

21. Li, J.; Koh, J. J.; Liu, S.; Lakshminarayanan, R.; Verma, C. S.; Beuerman, R. W., Membrane Active Antimicrobial Peptides: Translating Mechanistic Insights to Design. Front Neurosci 2017, 11, 73.

22. World Health Organization Ebola virus disease. http://www.who.int/en/newsroom/fact-sheets/detail/ebola-virus-disease.

23. Sanford, C. A.; West, T. E.; Jacob, S. T., Chapter 28 - Ebola Virus Disease and Hemorrhagic Fevers. In The Travel and Tropical Medicine Manual (Fifth Edition), Sanford, C. A.; Pottinger, P. S.; Jong, E. C., Eds. Elsevier: 2017; pp 391-400.

24. Osterholm, M. T.; Moore, K. A.; Kelley, N. S.; Brosseau, L. M.; Wong, G.; Murphy, F. A.; Peters, C. J.; LeDuc, J. W.; Russell, P. K.; Van Herp, M.; Kapetshi, J.; Muyembe, J. J.; Ilunga, B. K.; Strong, J. E.; Grolla, A.; Wolz, A.; Kargbo, B.; Kargbo, D. K.; Sanders, D. A.; Kobinger, G. P., Transmission of Ebola viruses: what we know and what we do not know. MBio 2015, 6 (2), e00137.

25. Adams, M. J.; Lefkowitz, E. J.; King, A. M.; Harrach, B.; Harrison, R. L.; Knowles, N. J.; Kropinski, A. M.; Krupovic, M.; Kuhn, J. H.; Mushegian, A. R.; Nibert, M.; Sabanadzovic, S.; Sanfacon, H.; Siddell, S. G.; Simmonds, P.; Varsani, A.; Zerbini, F. M.; Gorbalenya, A. E.; Davison, A. J., Ratification vote on taxonomic proposals to the International Committee on Taxonomy of Viruses (2016). Arch Virol 2016, 161 (10), 2921-49. 
26. Mari Saez, A.; Weiss, S.; Nowak, K.; Lapeyre, V.; Zimmermann, F.; Dux, A.; Kuhl, H. S.; Kaba, M.; Regnaut, S.; Merkel, K.; Sachse, A.; Thiesen, U.; Villanyi, L.; Boesch, C.; Dabrowski, P. W.; Radonic, A.; Nitsche, A.; Leendertz, S. A.; Petterson, S.; Becker, S.; Krahling, V.; Couacy-Hymann, E.; Akoua-Koffi, C.; Weber, N.; Schaade, L.; Fahr, J.; Borchert, M.; Gogarten, J. F.; Calvignac-Spencer, S.; Leendertz, F. H., Investigating the zoonotic origin of the West African Ebola epidemic. EMBO Mol Med 2015, 7 (1), 17-23.

27. Bwaka, M. A.; Bonnet, M. J.; Calain, P.; Colebunders, R.; De Roo, A.; Guimard, Y.; Katwiki, K. R.; Kibadi, K.; Kipasa, M. A.; Kuvula, K. J.; Mapanda, B. B.; Massamba, M.; Mupapa, K. D.; Muyembe-Tamfum, J. J.; Ndaberey, E.; Peters, C. J.; Rollin, P. E.; Van den Enden, E.; Van den Enden, E., Ebola hemorrhagic fever in Kikwit, Democratic Republic of the Congo: clinical observations in 103 patients. $J$ Infect Dis 1999, 179 Suppl 1, S1-7.

28. Kuhn, J. H., Filoviruses. A compendium of 40 years of epidemiological, clinical, and laboratory studies. Arch Virol Suppl 2008, 20, 13-360.

29. Pokhrel, R.; Pavadai, E.; Gerstman, B. S.; Chapagain, P. P., Membrane pore formation and ion selectivity of the Ebola virus delta peptide. Phys Chem Chem Phys 2019, 21 (10), 5578-5585.

30. Gallaher, W. R.; Garry, R. F., Modeling of the Ebola virus delta peptide reveals a potential lytic sequence motif. Viruses 2015, 7 (1), 285-305.

31. Gonzalez, M. E.; Carrasco, L., Viroporins. FEBS Lett 2003, 552 (1), 28-34.

32. Yount, N. Y.; Yeaman, M. R., Multidimensional signatures in antimicrobial peptides. Proc Natl Acad Sci U S A 2004, 101 (19), 7363-8.

33. Wimley, W. C., Describing the mechanism of antimicrobial peptide action with the interfacial activity model. ACS Chem Biol 2010, 5 (10), 905-17.

34. OuYang, B.; Chou, J. J., The minimalist architectures of viroporins and their therapeutic implications. Biochim Biophys Acta 2014, 1838 (4), 1058-67.

35. Nieto-Torres, J. L.; Verdia-Baguena, C.; Castano-Rodriguez, C.; Aguilella, V. M.; Enjuanes, L., Relevance of Viroporin Ion Channel Activity on Viral Replication and Pathogenesis. Viruses 2015, 7 (7), 3552-73.

36. Carrasco, L., Modification of membrane permeability by animal viruses. Adv Virus Res 1995, 45, 61-112.

37. Volchkova, V. A.; Feldmann, H.; Klenk, H. D.; Volchkov, V. E., The nonstructural small glycoprotein sGP of Ebola virus is secreted as an antiparallel-orientated homodimer. Virology 1998, 250 (2), 408-14. 
38. Volchkova, V. A.; Klenk, H. D.; Volchkov, V. E., Delta-peptide is the carboxyterminal cleavage fragment of the nonstructural small glycoprotein sGP of Ebola virus. Virology 1999, 265 (1), 164-71.

39. Hyser, J. M.; Utama, B.; Crawford, S. E.; Estes, M. K., Genetic divergence of rotavirus nonstructural protein 4 results in distinct serogroup-specific viroporin activity and intracellular punctate structure morphologies. J Virol 2012, 86 (9), 492134.

40. Yue, T.; Sun, M.; Zhang, S.; Ren, H.; Ge, B.; Huang, F., How transmembrane peptides insert and orientate in biomembranes: a combined experimental and simulation study. Phys Chem Chem Phys 2016, 18 (26), 17483-94.

41. He, J.; Melnik, L.; Komin, A.; Starr, C. G.; Fuselier, T.; Wiedman, G.; Morris, C. F.; Wang, Y.; Hristova, K.; Gallaher, W.; Garry, R. F.; Wimley, W. C., The Delta Peptide of Ebola Virus has Potent Viroporin Activity. Biophysical Journal 2017, 112 (3, Supplement 1), 185a.

42. Landers, T. F.; Cohen, B.; Wittum, T. E.; Larson, E. L., A review of antibiotic use in food animals: perspective, policy, and potential. Public Health Rep 2012, 127 (1), 422 .

43. Tangcharoensathien, V.; Chanvatik, S.; Sommanustweechai, A., Complex determinants of inappropriate use of antibiotics. Bull World Health Organ 2018, 96 (2), 141-144.

44. Ventola, C. L., The antibiotic resistance crisis: part 1: causes and threats. $P T \mathbf{2 0 1 5}$, $40(4), 277-83$.

45. Thabit, A. K.; Crandon, J. L.; Nicolau, D. P., Antimicrobial resistance: impact on clinical and economic outcomes and the need for new antimicrobials. Expert Opin Pharmacother 2015, 16 (2), 159-77.

46. World Health Organization Antibiotic resistance. https://www.who.int/newsroom/fact-sheets/detail/antibiotic-resistance (accessed 5 Feb).

47. Kritsotakis, E. I.; Kontopidou, F.; Astrinaki, E.; Roumbelaki, M.; Ioannidou, E.; Gikas, A., Prevalence, incidence burden, and clinical impact of healthcare-associated infections and antimicrobial resistance: a national prevalent cohort study in acute care hospitals in Greece. Infect Drug Resist 2017, 10, 317-328.

48. Karakonstantis, S.; Kritsotakis, E. I.; Gikas, A., Pandrug-resistant Gram-negative bacteria: a systematic review of current epidemiology, prognosis and treatment options. J Antimicrob Chemother 2020, 75 (2), 271-282.

49. Piddock, L. J. V., Reflecting on the final report of the O'Neill Review on Antimicrobial Resistance. Lancet Infect Dis 2016, 16 (7), 767-768. 
50. Rossolini, G. M.; Arena, F.; Pecile, P.; Pollini, S., Update on the antibiotic resistance crisis. Curr Opin Pharmacol 2014, 18, 56-60.

51. Ling, L. L.; Schneider, T.; Peoples, A. J.; Spoering, A. L.; Engels, I.; Conlon, B. P.; Mueller, A.; Schaberle, T. F.; Hughes, D. E.; Epstein, S.; Jones, M.; Lazarides, L.; Steadman, V. A.; Cohen, D. R.; Felix, C. R.; Fetterman, K. A.; Millett, W. P.; Nitti, A. G.; Zullo, A. M.; Chen, C.; Lewis, K., A new antibiotic kills pathogens without detectable resistance. Nature 2015, 517 (7535), 455-9.

52. Sahl, H. G.; Bierbaum, G., Lantibiotics: biosynthesis and biological activities of uniquely modified peptides from gram-positive bacteria. Annu Rev Microbiol 1998, $52,41-79$.

53. Rajeshkumar, N. V.; Kers, J. A.; Moncrief, S.; Defusco, A. W.; Park, J. H.; Handfield, M., Preclinical evaluation of the maximum tolerated dose and toxicokinetics of enteric-coated lantibiotic OG253 capsules. Toxicol Appl Pharmacol 2019, 374, 3240 .

54. Smith, L.; Zachariah, C.; Thirumoorthy, R.; Rocca, J.; Novak, J.; Hillman, J. D.; Edison, A. S., Structure and dynamics of the lantibiotic mutacin 1140. Biochemistry 2003, 42 (35), 10372-84.

55. Kirichenko, K.; Hillman, J. D.; Handfield, M.; Park, J. H., Complete synthesis of the bicyclic ring of a mutacin analog with orthogonally protected lanthionine via solidphase intracyclization. Journal of Peptide Science 2019, 25 (11), e3214.

56. Arnison, P. G.; Bibb, M. J.; Bierbaum, G.; Bowers, A. A.; Bugni, T. S.; Bulaj, G.; Camarero, J. A.; Campopiano, D. J.; Challis, G. L.; Clardy, J.; Cotter, P. D.; Craik, D. J.; Dawson, M.; Dittmann, E.; Donadio, S.; Dorrestein, P. C.; Entian, K. D.; Fischbach, M. A.; Garavelli, J. S.; Goransson, U.; Gruber, C. W.; Haft, D. H.; Hemscheidt, T. K.; Hertweck, C.; Hill, C.; Horswill, A. R.; Jaspars, M.; Kelly, W. L.; Klinman, J. P.; Kuipers, O. P.; Link, A. J.; Liu, W.; Marahiel, M. A.; Mitchell, D. A.; Moll, G. N.; Moore, B. S.; Muller, R.; Nair, S. K.; Nes, I. F.; Norris, G. E.; Olivera, B. M.; Onaka, H.; Patchett, M. L.; Piel, J.; Reaney, M. J.; Rebuffat, S.; Ross, R. P.; Sahl, H. G.; Schmidt, E. W.; Selsted, M. E.; Severinov, K.; Shen, B.; Sivonen, K.; Smith, L.; Stein, T.; Sussmuth, R. D.; Tagg, J. R.; Tang, G. L.; Truman, A. W.; Vederas, J. C.; Walsh, C. T.; Walton, J. D.; Wenzel, S. C.; Willey, J. M.; van der Donk, W. A., Ribosomally synthesized and post-translationally modified peptide natural products: overview and recommendations for a universal nomenclature. Nat Prod Rep 2013, 30 (1), 108-60.

57. Chatterjee, C.; Miller, L. M.; Leung, Y. L.; Xie, L.; Yi, M.; Kelleher, N. L.; van der Donk, W. A., Lacticin 481 synthetase phosphorylates its substrate during lantibiotic production. J Am Chem Soc 2005, 127 (44), 15332-3. 
58. Goto, Y.; Li, B.; Claesen, J.; Shi, Y.; Bibb, M. J.; van der Donk, W. A., Discovery of unique lanthionine synthetases reveals new mechanistic and evolutionary insights. PLoS Biol 2010, 8 (3), e1000339.

59. Willey, J. M.; van der Donk, W. A., Lantibiotics: peptides of diverse structure and function. Annu Rev Microbiol 2007, 61, 477-501.

60. Munch, D.; Sahl, H. G., Structural variations of the cell wall precursor lipid II in Gram-positive bacteria - Impact on binding and efficacy of antimicrobial peptides. Biochim Biophys Acta 2015, 1848 (11 Pt B), 3062-71.

61. Hsu, S. T.; Breukink, E.; Tischenko, E.; Lutters, M. A.; de Kruijff, B.; Kaptein, R.; Bonvin, A. M.; van Nuland, N. A., The nisin-lipid II complex reveals a pyrophosphate cage that provides a blueprint for novel antibiotics. Nat Struct Mol Biol 2004, 11 (10), 963-7.

62. van Heusden, H. E.; de Kruijff, B.; Breukink, E., Lipid II induces a transmembrane orientation of the pore-forming peptide lantibiotic nisin. Biochemistry 2002, 41 (40), 12171-8.

63. Bonelli, R. R.; Schneider, T.; Sahl, H. G.; Wiedemann, I., Insights into in vivo activities of lantibiotics from gallidermin and epidermin mode-of-action studies. Antimicrob Agents Chemother 2006, 50 (4), 1449-57.

64. Hasper, H. E.; de Kruijff, B.; Breukink, E., Assembly and stability of nisin-lipid II pores. Biochemistry 2004, 43 (36), 11567-75.

65. Oppedijk, S. F.; Martin, N. I.; Breukink, E., Hit 'em where it hurts: The growing and structurally diverse family of peptides that target lipid-II. Biochimica et Biophysica Acta (BBA) - Biomembranes 2016, 1858 (5), 947-957.

66. Draper, L. A.; Cotter, P. D.; Hill, C.; Ross, R. P., Lantibiotic resistance. Microbiol Mol Biol Rev 2015, 79 (2), 171-91.

67. Khosa, S.; Frieg, B.; Mulnaes, D.; Kleinschrodt, D.; Hoeppner, A.; Gohlke, H.; Smits, S. H., Structural basis of lantibiotic recognition by the nisin resistance protein from Streptococcus agalactiae. Sci Rep 2016, 6, 18679.

68. Gardete, S.; Tomasz, A., Mechanisms of vancomycin resistance in Staphylococcus aureus. J Clin Invest 2014, 124 (7), 2836-40.

69. Sandiford, S. K., Perspectives on lantibiotic discovery - where have we failed and what improvements are required? Expert Opinion on Drug Discovery 2015, 10 (4), 315-320.

70. Mulholland, S.; Turpin, E. R.; Bonev, B. B.; Hirst, J. D., Docking and molecular dynamics simulations of the ternary complex nisin2:lipid II. Sci Rep 2016, 6, 21185. 
71. Bauer, R.; Dicks, L. M., Mode of action of lipid II-targeting lantibiotics. Int J Food Microbiol 2005, 101 (2), 201-16.

72. Pokhrel, R.; Bhattarai, N.; Baral, P.; Gerstman, B. S.; Park, J. H.; Handfield, M.; Chapagain, P. P., Molecular mechanisms of pore formation and membrane disruption by the antimicrobial lantibiotic peptide Mutacin 1140. Phys Chem Chem Phys 2019, 21 (23), 12530-12539.

73. Tomar, S. K.; Knauer, S. H.; NandyMazumdar, M.; Rösch, P.; Artsimovitch, I., Interdomain contacts control folding of transcription factor RfaH. Nucleic acids research 2013, 41 (22), 10077-10085.

74. Bačová, P.; Rissanou, A. N.; Harmandaris, V., Modelling of novel polymer materials through atomistic molecular dynamics simulations. Procedia Computer Science 2018, 136, 341-350.

75. De Vivo, M.; Masetti, M.; Bottegoni, G.; Cavalli, A., Role of Molecular Dynamics and Related Methods in Drug Discovery. J Med Chem 2016, 59 (9), 4035-61.

76. Phillips, J. C.; Braun, R.; Wang, W.; Gumbart, J.; Tajkhorshid, E.; Villa, E.; Chipot, C.; Skeel, R. D.; Kale, L.; Schulten, K., Scalable molecular dynamics with NAMD. J Comput Chem 2005, 26 (16), 1781-802.

77. Huang, J.; Rauscher, S.; Nawrocki, G.; Ran, T.; Feig, M.; de Groot, B. L.; Grubmuller, H.; MacKerell, A. D., Jr., CHARMM36m: an improved force field for folded and intrinsically disordered proteins. Nat Methods 2017, 14 (1), 71-73.

78. Turpin, E. R.; Mulholland, S.; Teale, A. M.; Bonev, B. B.; Hirst, J. D., New CHARMM force field parameters for dehydrated amino acid residues, the key to lantibiotic molecular dynamics simulations. RSC Advances 2014, 4 (89), 4862148631.

79. Wu, E. L.; Cheng, X.; Jo, S.; Rui, H.; Song, K. C.; Davila-Contreras, E. M.; Qi, Y.; Lee, J.; Monje-Galvan, V.; Venable, R. M.; Klauda, J. B.; Im, W., CHARMM-GUI Membrane Builder toward realistic biological membrane simulations. J Comput Chem 2014, 35 (27), 1997-2004.

80. Zachowski, A., Phospholipids in animal eukaryotic membranes: transverse asymmetry and movement. Biochem J 1993, 294 ( Pt 1), 1-14.

81. Gc, J. B.; Gerstman, B. S.; Stahelin, R. V.; Chapagain, P. P., The Ebola virus protein VP40 hexamer enhances the clustering of PI(4,5)P2 lipids in the plasma membrane. Phys Chem Chem Phys 2016, 18 (41), 28409-28417.

82. Gc, J. B.; Gerstman, B. S.; Chapagain, P. P., Membrane association and localization dynamics of the Ebola virus matrix protein VP40. Biochim Biophys Acta 2017, 1859 (10), 2012-2020. 
83. Bhattarai, N.; Gc, J. B.; Gerstman, B. S.; Stahelin, R. V.; Chapagain, P. P., Plasma membrane association facilitates conformational changes in the Marburg virus protein VP40 dimer. $R S C A d v \mathbf{2 0 1 7}, 7$ (37), 22741-22748.

84. Chugunov, A.; Pyrkova, D.; Nolde, D.; Polyansky, A.; Pentkovsky, V.; Efremov, R., Lipid-II forms potential "landing terrain" for lantibiotics in simulated bacterial membrane. Sci Rep 2013, 3, 1678.

85. Essmann, U.; Perera, L.; Berkowitz, M. L.; Darden, T.; Lee, H.; Pedersen, L. G., A smooth particle mesh Ewald method. The Journal of Chemical Physics 1995, 103 (19), 8577-8593.

86. Harvey, M. J.; De Fabritiis, G., An Implementation of the Smooth Particle Mesh Ewald Method on GPU Hardware. J Chem Theory Comput 2009, 5 (9), 2371-7.

87. Ryckaert, J.-P.; Ciccotti, G.; Berendsen, H. J. C., Numerical integration of the cartesian equations of motion of a system with constraints: molecular dynamics of nalkanes. Journal of Computational Physics 1977, 23 (3), 327-341.

88. Humphrey, W.; Dalke, A.; Schulten, K., VMD: visual molecular dynamics. $J$ Mol Graph 1996, 14 (1), 33-8, 27-8.

89. Lu, H.; Schulten, K., Steered molecular dynamics simulations of force-induced protein domain unfolding. Proteins 1999, 35 (4), 453-63.

90. Zheng, L.; Alhossary, A. A.; Kwoh, C.-K.; Mu, Y., Molecular Dynamics and Simulation. In Encyclopedia of Bioinformatics and Computational Biology, Ranganathan, S.; Gribskov, M.; Nakai, K.; Schönbach, C., Eds. Academic Press: Oxford, 2019; pp 550-566.

91. Comer, J.; Gumbart, J. C.; Hénin, J.; Lelièvre, T.; Pohorille, A.; Chipot, C., The Adaptive Biasing Force Method: Everything You Always Wanted To Know but Were Afraid To Ask. The Journal of Physical Chemistry B 2015, 119 (3), 1129-1151.

92. Kumar, S.; Rosenberg, J. M.; Bouzida, D.; Swendsen, R. H.; Kollman, P. A., THE weighted histogram analysis method for free-energy calculations on biomolecules. I. The method. Journal of Computational Chemistry 1992, 13 (8), 1011-1021.

93. Prince, A.; Sandhu, P.; Ror, P.; Dash, E.; Sharma, S.; Arakha, M.; Jha, S.; Akhter, Y.; Saleem, M., Lipid-II Independent Antimicrobial Mechanism of Nisin Depends On Its Crowding And Degree Of Oligomerization. Sci Rep 2016, 6, 37908.

94. Kelley, L. A.; Mezulis, S.; Yates, C. M.; Wass, M. N.; Sternberg, M. J., The Phyre2 web portal for protein modeling, prediction and analysis. Nat Protoc 2015, 10 (6), 845-58. 
95. Smart, O. S.; Neduvelil, J. G.; Wang, X.; Wallace, B. A.; Sansom, M. S., HOLE: a program for the analysis of the pore dimensions of ion channel structural models. $J$ Mol Graph 1996, 14 (6), 354-60, 376.

96. Chen, H.; Wu, Y.; Voth, G. A., Proton transport behavior through the influenza A M2 channel: insights from molecular simulation. Biophys $J$ 2007, 93 (10), 3470-9.

97. Sula, A.; Wallace, B. A., Interpreting the functional role of a novel interaction motif in prokaryotic sodium channels. J Gen Physiol 2017, 149 (6), 613-622.

98. Laghaei, R.; Kowallis, W.; Evans, D. G.; Coalson, R. D., Calculation of iron transport through human H-chain ferritin. J Phys Chem A 2014, 118 (35), 7442-53.

99. Giorgino, T., Computing 1-D atomic densities in macromolecular simulations: The density profile tool for VMD. Computer Physics Communications 2014, 185 (1), 317-322.

100. He, J.; Melnik, L. I.; Komin, A.; Wiedman, G.; Fuselier, T.; Morris, C. F.; Starr, C. G.; Searson, P. C.; Gallaher, W. R.; Hristova, K.; Garry, R. F.; Wimley, W. C., Ebola Virus Delta Peptide Is a Viroporin. J Virol 2017, 91 (16).

101. Zhao, B. C.; Lin, H. C.; Yang, D.; Ye, X.; Li, Z. G., Disulfide Bridges in Defensins. Curr Top Med Chem 2015, 16 (2), 206-19.

102. Hasper, H. E.; Kramer, N. E.; Smith, J. L.; Hillman, J. D.; Zachariah, C.; Kuipers, O. P.; de Kruijff, B.; Breukink, E., An alternative bactericidal mechanism of action for lantibiotic peptides that target lipid II. Science 2006, 313 (5793), 1636-7.

103. Schwartz, B.; Markwalder, J. A.; Wang, Y., Lipid II: total synthesis of the bacterial cell wall precursor and utilization as a substrate for glycosyltransfer and transpeptidation by penicillin binding protein (PBP) 1 b of Escherichia coli. $J \mathrm{Am}$ Chem Soc 2001, 123 (47), 11638-43.

104. Bahar, A. A.; Ren, D., Antimicrobial peptides. Pharmaceuticals (Basel) 2013, 6 (12), 1543-75.

105. Medeiros-Silva, J.; Jekhmane, S.; Paioni, A. L.; Gawarecka, K.; Baldus, M.; Swiezewska, E.; Breukink, E.; Weingarth, M., High-resolution NMR studies of antibiotics in cellular membranes. Nat Commun 2018, 9 (1), 3963.

106. Breukink, E.; de Kruijff, B., Lipid II as a target for antibiotics. Nat Rev Drug Discov 2006, 5 (4), 321-32.

107. Christ, K.; Al-Kaddah, S.; Wiedemann, I.; Rattay, B.; Sahl, H. G.; Bendas, G., Membrane lipids determine the antibiotic activity of the lantibiotic gallidermin. $J$ Membr Biol 2008, 226 (1-3), 9-16. 
108. Qi, Y.; Cheng, X.; Lee, J.; Vermaas, J. V.; Pogorelov, T. V.; Tajkhorshid, E.; Park, S.; Klauda, J. B.; Im, W., CHARMM-GUI HMMM Builder for Membrane Simulations with the Highly Mobile Membrane-Mimetic Model. Biophys J 2015, 109 (10), 2012-22.

109. Kofinger, J.; Hummer, G.; Dellago, C., Single-file water in nanopores. Phys Chem Chem Phys 2011, 13 (34), 15403-17.

110. Hummer, G.; Rasaiah, J. C.; Noworyta, J. P., Water conduction through the hydrophobic channel of a carbon nanotube. Nature 2001, 414 (6860), 188-90.

111. Pomes, R.; Roux, B., Molecular mechanism of $\mathrm{H}+$ conduction in the single-file water chain of the gramicidin channel. Biophys $J$ 2002, 82 (5), 2304-16.

112. Dani, J. A.; Levitt, D. G., Water transport and ion-water interaction in the gramicidin channel. Biophys J 1981, 35 (2), 501-8.

113. Allen, T. W.; Andersen, O. S.; Roux, B., Energetics of ion conduction through the gramicidin channel. Proc Natl Acad Sci U S A 2004, 101 (1), 117-22.

114. Bastug, T.; Kuyucak, S., Energetics of ion permeation, rejection, binding, and block in gramicidin A from free energy simulations. Biophys J 2006, 90 (11), 3941-50.

115. Agre, P., Aquaporin Water Channels (Nobel Lecture). Angewandte Chemie International Edition 2004, 43 (33), 4278-4290.

116. Kers, J. A.; Sharp, R. E.; Muley, S.; Mayo, M.; Colbeck, J.; Zhu, Y.; DeFusco, A. W.; Park, J. H.; Handfield, M., Blueprints for the rational design of therapeutic mutacin 1140 variants. Chem Biol Drug Des 2018, 92 (6), 1940-1953.

117. Horner, A.; Zocher, F.; Preiner, J.; Ollinger, N.; Siligan, C.; Akimov, S. A.; Pohl, P., The mobility of single-file water molecules is governed by the number of $\mathrm{H}$-bonds they may form with channel-lining residues. Sci Adv 2015, 1 (2), e1400083.

118. van Hijkoop, V. J.; Dammers, A. J.; Malek, K.; Coppens, M. O., Water diffusion through a membrane protein channel: a first passage time approach. $J$ Chem Phys 2007, 127 (8), 085101.

119. Chiu, S. W.; Subramaniam, S.; Jakobsson, E., Simulation study of a gramicidin/lipid bilayer system in excess water and lipid. II. Rates and mechanisms of water transport. Biophys J 1999, 76 (4), 1939-50.

120. Lee, M. T.; Hung, W. C.; Chen, F. Y.; Huang, H. W., Mechanism and kinetics of pore formation in membranes by water-soluble amphipathic peptides. Proc Natl Acad Sci U S A 2008, 105 (13), 5087-92. 
121. Medeiros-Silva, J.; Jekhmane, S.; Breukink, E.; Weingarth, M., Towards the native binding modes of Lipid II targeting antibiotics. ChemBioChem 2019, 0 (ja).

122. Gross, E.; Morell, J. L., The structure of nisin. J Am Chem Soc 1971, 93 (18), 46345.

123. Gotz, F.; Perconti, S.; Popella, P.; Werner, R.; Schlag, M., Epidermin and gallidermin: Staphylococcal lantibiotics. Int J Med Microbiol 2014, 304 (1), 63-71.

124. Petrosillo, N.; Granata, G.; Cataldo, M. A., Novel Antimicrobials for the Treatment of Clostridium difficile Infection. Front Med (Lausanne) 2018, 5, 96.

125. Munch, D.; Muller, A.; Schneider, T.; Kohl, B.; Wenzel, M.; Bandow, J. E.; Maffioli, S.; Sosio, M.; Donadio, S.; Wimmer, R.; Sahl, H. G., The lantibiotic NAI-107 binds to bactoprenol-bound cell wall precursors and impairs membrane functions. $J$ Biol Chem 2014, 289 (17), 12063-76.

126. Crowther, G. S.; Baines, S. D.; Todhunter, S. L.; Freeman, J.; Chilton, C. H.; Wilcox, M. H., Evaluation of NVB302 versus vancomycin activity in an in vitro human gut model of Clostridium difficile infection. J Antimicrob Chemother 2013, 68 (1), 16876.

127. Boakes, S.; Dawson, M. J., Discovery and Development of NVB302, a Semisynthetic Antibiotic for Treatment of Clostridium difficile Infection. Natural Products 2014, 455-468.

128. van Kraaij, C.; Breukink, E.; Noordermeer, M. A.; Demel, R. A.; Siezen, R. J.; Kuipers, O. P.; de Kruijff, B., Pore formation by nisin involves translocation of its Cterminal part across the membrane. Biochemistry 1998, 37 (46), 16033-40.

129. Horner, A.; Pohl, P., Single-file transport of water through membrane channels. Faraday Discuss 2018, 209 (0), 9-33.

130. Breukink, E.; de Kruijff, B., Lipid II as a target for antibiotics. Nature Reviews Drug Discovery 2006, 5 (4), 321-323.

131. Ventola, C. L., The antibiotic resistance crisis: part 2: management strategies and new agents. $P T$ 2015, 40 (5), 344-52.

132. Silver, L. L., Challenges of antibacterial discovery. Clin Microbiol Rev 2011, 24 (1), 71-109.

133. Singh, S. B., Confronting the challenges of discovery of novel antibacterial agents. Bioorg Med Chem Lett 2014, 24 (16), 3683-9.

134. Parkes, A. L.; Yule, I. A., Hybrid antibiotics - clinical progress and novel designs. Expert Opin Drug Discov 2016, 11 (7), 665-80. 
135. Barbachyn, M. R., Chapter 17 - Recent Advances in the Discovery of Hybrid Antibacterial Agents. In Annual Reports in Medicinal Chemistry, Macor, J. E., Ed. Academic Press: 2008; Vol. 43, pp 281-290.

136. Pokrovskaya, V.; Baasov, T., Dual-acting hybrid antibiotics: a promising strategy to combat bacterial resistance. Expert Opin Drug Discov 2010, 5 (9), 883-902.

137. Tevyashova, A. N.; Olsufyeva, E. N.; Preobrazhenskaya, M. N., Design of dual action antibiotics as an approach to search for new promising drugs. Russian Chemical Reviews 2015, 84 (1), 61-97.

138. Cebrian, R.; Macia-Valero, A.; Jati, A. P.; Kuipers, O. P., Design and Expression of Specific Hybrid Lantibiotics Active Against Pathogenic Clostridium spp. Front Microbiol 2019, 10, 2154.

139. Geng, M.; Smith, L., Improving the attrition rate of Lanthipeptide discovery for commercial applications. Expert Opin Drug Discov 2018, 13 (2), 155-167.

140. Wilson-Stanford, S.; Kalli, A.; Hakansson, K.; Kastrantas, J.; Orugunty, R. S.; Smith, L., Oxidation of lanthionines renders the lantibiotic nisin inactive. Appl Environ Microbiol 2009, 75 (5), 1381-7.

141. Smith, L.; Hillman, J., Therapeutic potential of type A (I) lantibiotics, a group of cationic peptide antibiotics. Curr Opin Microbiol 2008, 11 (5), 401-8.

142. Sit, C. S.; Yoganathan, S.; Vederas, J. C., Biosynthesis of aminovinyl-cysteinecontaining peptides and its application in the production of potential drug candidates. Acc Chem Res 2011, 44 (4), 261-8.

143. Escano, J.; Ravichandran, A.; Salamat, B.; Smith, L., Carboxyl Analogue of Mutacin 1140, a Scaffold for Lead Antibacterial Discovery. Appl Environ Microbiol 2017, 83 (14).

144. Escano, J.; Smith, L., Multipronged approach for engineering novel peptide analogues of existing lantibiotics. Expert Opin Drug Discov 2015, 10 (8), 857-70.

145. Pushpanathan, M.; Gunasekaran, P.; Rajendhran, J., Antimicrobial peptides: versatile biological properties. Int J Pept 2013, 2013, 675391.

146. Role of Disulfide Bonds in Peptide and Protein Conformation. In Amino Acids, Peptides and Proteins in Organic Chemistry, pp 395-417.

147. Patel, R. R.; Chaurasia, S.; Khan, G.; Chaubey, P.; Kumar, N.; Mishra, B., Highly water-soluble mast cell stabiliser-encapsulated solid lipid nanoparticles with enhanced oral bioavailability. J Microencapsul 2016, 33 (3), 209-20. 
148. Hart, M. L.; Do, D. P.; Ansari, R. A.; Rizvi, S. A. A. In Brief Overview of Various Approaches to Enhance Drug Solubility, 2013.

149. Savjani, K. T.; Gajjar, A. K.; Savjani, J. K., Drug solubility: importance and enhancement techniques. ISRN Pharm 2012, 2012, 195727.

150. Kalepu, S.; Nekkanti, V., Insoluble drug delivery strategies: review of recent advances and business prospects. Acta Pharm Sin B 2015, 5 (5), 442-53.

151. Acharya, P. C.; Fernandes, C.; Suares, D.; Shetty, S.; Tekade, R. K., Chapter 15 Solubility and Solubilization Approaches in Pharmaceutical Product Development. In Dosage Form Design Considerations, Tekade, R. K., Ed. Academic Press: 2018; pp 513-547.

152. Kapoor, D.; Maheshwari, R.; Verma, K.; Sharma, S.; Pethe, A.; Tekade, R. K., Chapter 1 - Fundamentals of diffusion and dissolution: dissolution testing of pharmaceuticals. In Drug Delivery Systems, Tekade, R. K., Ed. Academic Press: 2020; pp 1-45.

153. Yang, W.; Gadgil, P.; Krishnamurthy, V. R.; Landis, M.; Mallick, P.; Patel, D.; Patel, P. J.; Reid, D. L.; Sanchez-Felix, M., The Evolving Druggability and Developability Space: Chemically Modified New Modalities and Emerging Small Molecules. AAPS J 2020, 22 (2), 21.

154. Krishnaiah, Y. S. R., Pharmaceutical Technologies for Enhancing Oral Bioavailability of Poorly Soluble Drugs. Journal of Bioequivalence \& Bioavailability 2010, 2, 1-5.

155. Simeonov, P.; Berger-Hoffmann, R.; Hoffmann, R.; Strater, N.; Zuchner, T., Surface supercharged human enteropeptidase light chain shows improved solubility and refolding yield. Protein Eng Des Sel 2011, 24 (3), 261-8.

156. Sarma, R.; Wong, K.-Y.; Lynch, G. C.; Pettitt, B. M., Peptide Solubility Limits: Backbone and Side-Chain Interactions. The Journal of Physical Chemistry B 2018, 122 (13), 3528-3539.

157. Patel, A. J.; Varilly, P.; Chandler, D., Fluctuations of water near extended hydrophobic and hydrophilic surfaces. J Phys Chem B 2010, 114 (4), 1632-7.

158. Rouse, S. L.; Sansom, M. S., Interactions of lipids and detergents with a viral ion channel protein: molecular dynamics simulation studies. J Phys Chem B 2015, 119 (3), 764-72.

159. de Kruijff, B.; van Dam, V.; Breukink, E., Lipid II: a central component in bacterial cell wall synthesis and a target for antibiotics. Prostaglandins Leukot Essent Fatty Acids 2008, 79 (3-5), 117-21. 
160. Breukink, E.; Wiedemann, I.; van Kraaij, C.; Kuipers, O. P.; Sahl, H. G.; de Kruijff, B., Use of the cell wall precursor lipid II by a pore-forming peptide antibiotic. Science 1999, 286 (5448), 2361-4.

161. Thomsen, T. T.; Mojsoska, B.; Cruz, J. C.; Donadio, S.; Jenssen, H.; Lobner-Olesen, A.; Rewitz, K., The Lantibiotic NAI-107 Efficiently Rescues Drosophila melanogaster from Infection with Methicillin-Resistant Staphylococcus aureus USA300. Antimicrob Agents Chemother 2016, 60 (9), 5427-36.

162. Saier, M. H., Jr.; Reddy, B. L., Holins in bacteria, eukaryotes, and archaea: multifunctional xenologues with potential biotechnological and biomedical applications. J Bacteriol 2015, 197 (1), 7-17.

163. Costin, J. M.; Rausch, J. M.; Garry, R. F.; Wimley, W. C., Viroporin potential of the lentivirus lytic peptide (LLP) domains of the HIV-1 gp41 protein. Virol J 2007, 4, 123.

164. Wimley, W. C.; Hristova, K., The Mechanism of Membrane Permeabilization by Peptides: Still an Enigma. Aust J Chem 2019, 73 (3), 96-103.

165. Henderson, J. M.; Waring, A. J.; Separovic, F.; Lee, K. Y. C., Antimicrobial Peptides Share a Common Interaction Driven by Membrane Line Tension Reduction. Biophys J 2016, 111 (10), 2176-2189.

166. Li, W.; Tailhades, J.; O'Brien-Simpson, N. M.; Separovic, F.; Otvos, L., Jr.; Hossain, M. A.; Wade, J. D., Proline-rich antimicrobial peptides: potential therapeutics against antibiotic-resistant bacteria. Amino Acids 2014, 46 (10), 2287-94.

167. Sani, M. A.; Whitwell, T. C.; Gehman, J. D.; Robins-Browne, R. M.; Pantarat, N.; Attard, T. J.; Reynolds, E. C.; O'Brien-Simpson, N. M.; Separovic, F., Maculatin 1.1 disrupts Staphylococcus aureus lipid membranes via a pore mechanism. Antimicrob Agents Chemother 2013, 57 (8), 3593-600.

168. Badani, H.; Garry, R. F.; Wimley, W. C., Peptide entry inhibitors of enveloped viruses: the importance of interfacial hydrophobicity. Biochim Biophys Acta 2014, $1838(9), 2180-97$.

169. Sandhaus, S.; Chapagain, P. P.; Tse-Dinh, Y. C., Discovery of novel bacterial topoisomerase I inhibitors by use of in silico docking and in vitro assays. Sci Rep 2018, $8(1), 1437$.

170. Acedo, J. Z.; Chiorean, S.; Vederas, J. C.; van Belkum, M. J., The expanding structural variety among bacteriocins from Gram-positive bacteria. FEMS Microbiol Rev 2018, 42 (6), 805-828.

171. World Health Organization Ebola virus disease. http://www.who.int/mediacentre/factsheets/fs103/en/ (accessed 12 Feb). 
172. Wiedemann, I.; Breukink, E.; van Kraaij, C.; Kuipers, O. P.; Bierbaum, G.; de Kruijff, B.; Sahl, H. G., Specific binding of nisin to the peptidoglycan precursor lipid II combines pore formation and inhibition of cell wall biosynthesis for potent antibiotic activity. $J$ Biol Chem 2001, 276 (3), 1772-9.

173. Gut, I. M.; Blanke, S. R.; van der Donk, W. A., Mechanism of inhibition of Bacillus anthracis spore outgrowth by the lantibiotic nisin. ACS Chem Biol 2011, 6 (7), 74452.

174. Kers, J. A.; DeFusco, A. W.; Park, J. H.; Xu, J.; Pulse, M. E.; Weiss, W. J.; Handfield, M., OG716: Designing a fit-for-purpose lantibiotic for the treatment of Clostridium difficile infections. PLoS One 2018, 13 (6), e0197467.

175. Geng, M.; Smith, L., Modifying the Lantibiotic Mutacin 1140 for Increased Yield, Activity, and Stability. Appl Environ Microbiol 2018, 84 (15). 
VITA

RUDRAMANI POKHREL

\section{EDUCATION}

- Florida International University, Miami, FL, Ph.D. Physics, March 2020.

- Tribhuvan University, Kathmandu, Nepal, M. Sc., Physics, 2008.

- Tribhuvan University, Nepalgunj, Nepal, B. Sc., Physics, 2006.

\section{PUBLICATIONS:}

1. R. Pokhrel; Prem Chapagain; Jessica Siltberg-Liberles, Potential RNA-dependent RNA Polymerase inhibitors as prospective therapeutics against SARS-CoV-2, Journal of Medical Microbiology. doi:https://doi.org/10.1099/jmm.0.001203.

2. Kristen Johnson, R. Pokhrel, Melissa Budicini, Bernard Gerstman, Prem Chapagain, Robert Stahelin, A Conserved Tryptophan in the Ebola Virus Matrix Protein C-terminal domain is required for Efficient Virus-Like Particle Formation, Pathogens 2020, 9(5), 402

3. R. Pokhrel, N. Bhattarai, P. Baral, B.S. Gerstman, J.H. Park, M. Handfield, P.P. Chapagain, Molecular mechanisms of pore formation and membrane disruption by the antimicrobial lantibiotic peptide Mutacin 1140, Phys Chem Chem Phys, 21 (2019) 12530-12539.

4. R. Pokhrel, E. Pavadai, B.S. Gerstman, P.P. Chapagain, Membrane pore formation and ion selectivity of the Ebola virus delta peptide, Phys Chem Chem Phys, 21 (2019) 5578-5585.

5. R. Pokhrel, P. Sompornpisut, P. Chapagain, B. Olson, B. Gerstman, R.B. Pandey, Domain rearrangement and denaturation in Ebola virus protein VP40, AIP Advances, 8 (2018) 125129.

6. R. Pokhrel, B.S. Gerstman, J.D. Hutcheson, P.P. Chapagain, In Silico Investigations of Calcium Phosphate Mineralization in Extracellular Vesicles, J Phys Chem B, 122 (2018) 3782-3789. 
7. J.B. GC, R. Pokhrel, N. Bhattarai, K.A. Johnson, B.S. Gerstman, R.V. Stahelin, P.P. Chapagain, Graphene-VP40 interactions and potential disruption of the Ebola virus matrix filaments, Biochem Biophys Res Commun, 493 (2017) 176-181.

8. R. Pokhrel, E. Pavadai, B.S. Gerstman, P.P. Chapagain Ion Selective Pentameric Pore Formation by Ebola Virus Delta Peptide, Biophysical Society Meeting, San Diego, CA, Feb 15-19, 2020.

9. R. Pokhrel, B.S. Gerstman, J.D. Hutcheson, P.P. Chapagain, Computational Study of Calcium Phosphate Mineralization in Extracellular Vesicles, Biophysical Society Meeting, Baltimore, MD,2019, (Biophysical Journal, Volume 116, Issue 3).

10. R. Pokhrel, J.B. GC, N. Bhattarai, K.A. Johnson, B.S. Gerstman, R.V. Stahelin, P.P. Chapagain, Potential Disruption of Ebola Virus Matrix by Graphene NanoSheets, Biophysical Society Meeting, San Francisco, California, 2018, (Biophysical Journal, Volume 114, Issue 3). 